\title{
FLUID AGITATION STUDIES FOR DRUG PRODUCT CONTAINERS USING COMPUTATIONAL FLUID DYNAMICS (CFD)
}

\author{
A Thesis \\ presented to \\ the Faculty of California Polytechnic State University, \\ San Luis Obispo
}

\author{
In Partial Fulfillment \\ of the Requirements for the Degree \\ Master of Science in Mechanical Engineering
}

by

Matthew Ichinose

December 2018 
(C) 2018

Matthew Ichinose

ALL RIGHTS RESERVED 


\title{
COMMITTEE MEMBERSHIP
}

\author{
TITLE: Fluid Agitation Studies for Drug Product \\ Containers using Computational Fluid Dy- \\ namics (CFD)
}

AUTHOR: Matthew Ichinose

DATE SUBMITTED: December 2018

COMMITTEE CHAIR: Dr. Kim Shollenberger, Ph.D.

Professor of Mechanical Engineering

COMMITTEE MEMBER: Dr. Hans Mayer, Ph.D.

Lecturer of Mechanical Engineering

COMmitTeE MeMBER: Dr. Graham Milne, Ph.D.

Senior Scientist of Amgen Inc. 


\begin{abstract}
Fluid Agitation Studies for Drug Product Containers using Computational Fluid Dynamics (CFD)

Matthew Ichinose
\end{abstract}

At Amgen, the Automated Vision Inspection (AVI) systems capture the movement of unwanted particles in Amgen's drug product containers. For quality inspection, the AVI system must detect these undesired particles using a high speed spin-stop agitation process. To better understand the fluid movements to swirl the particles away from the walls, Computational Fluid Dynamics (CFD) is used to analyze the nature of the two phase flow of air and a liquid solution.

Several 2-D and 3-D models were developed using Fluent to create simulations of Amgen's drug product containers for a $1 \mathrm{~mL}$ syringe, $2.25 \mathrm{~mL}$ syringe, and a 5 $\mathrm{mL}$ cartridge. Fluid motion and potential bubble formations were studied within the liquid/gas domain inside the container by varying parameters such as viscosity, angular velocity, and surface tension. Experiments were conducted using Amgen's own equipment to capture the images of the spin-stop process and validate the models created in Fluent. Observations were made to see the effects of bubble formation or splashing during spin-down to rest.

The numerical and experimental results showed favorable comparison when measuring the meniscus height or the surface profile between the air and liquid. Also, at high angular velocity and dynamic viscosity, the container experiences instabilities and bubble formations. These studies indicate that CFD can be used as an useful and important tool to study fluid movement during agitation and observe any undesirable results for quality inspection. 


\section{ACKNOWLEDGMENTS}

I would like to thank all my committee members for their support throughout the process and helping me become a better student as an independent thinker who can overcome obstacles and learn from mistakes.

I would like to thank my thesis advisor, Dr. Shollenberger who took on this project and provided me invaluable insight and commitment to help me understand the intricacies of CFD. Dr. Shollenberger helped me formulate the thesis from the inception of the project and guided me throughout the process. I would like to thank Dr. Mayer for your help and advice throughout the process. It was in Dr. Mayer's fluid mechanics class that I found fluids to be so interesting yet challenging and fulfilling.

I would like to show my gratitude and thanks to Dr. Graham Milne and the TAO team for their support from my internship in the summer of 2016 to my completion of my thesis project. Graham, thank you for starting this project and also working hard to provide funding for this project. I truly appreciate the opportunities and doors you created for me to acquire knowledge about the biotechnology industry, the automated inspection process, and in life.

I would also like to acknowledge Alex Ramos from the Aerospace Engineering Department to use the Bishop High Performance Computing Cluster to run my simulations. Without their help, my simulations would be running for weeks and months instead of hours and days.

Finally, I would like to express my gratitude to my friends and family who helped me get this far to complete my degree in engineering after my career change from architecture to engineering. I want to especially thank my wife, Cyndi, who always encouraged me to follow my goals and be who I am. Despite living apart, thank you for your unconditional support and love for me for near and far. 


\section{TABLE OF CONTENTS}

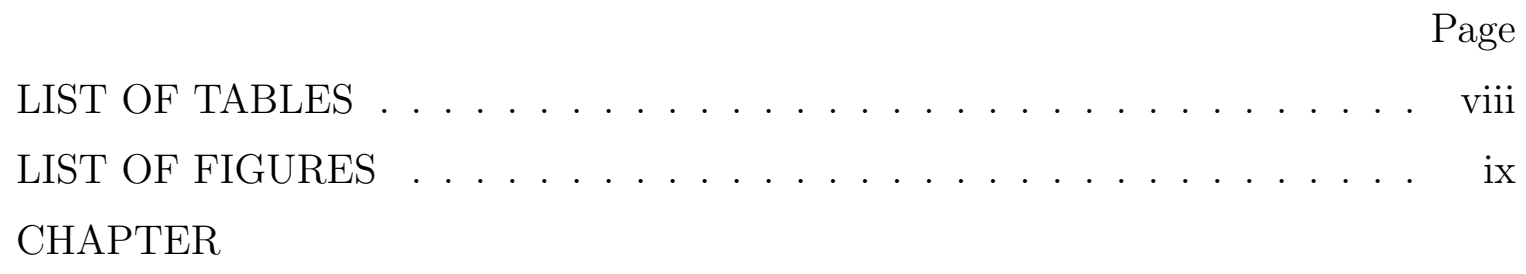

1 Introduction . . . . . . . . . . . . . . . . . 1

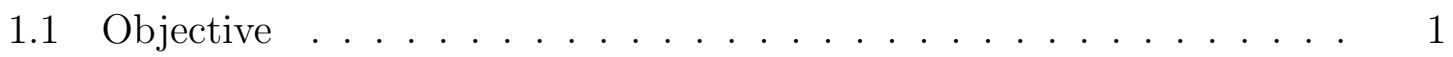

1.2 Motivation . . . . . . . . . . . . . . . . . 1

1.3 Previous Work ....................... 4

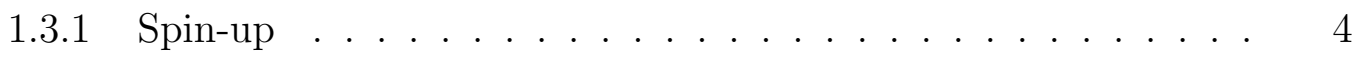

1.3.1.1 Liquid Filled Container .......... 6

1.3.1.2 Partially-Filled Liquid Container . . . . . . . . . 9

1.3.2 Steady State Meniscus . . . . . . . . . . . . . . . . 12

1.3.3 Spin-down to Rest . . . . . . . . . . . . . 13

1.3.3.1 Instabilities and Effects of Taylor-Gortler Vortices . . 14

1.3.3.2 Bubble Formation during Spin-Down . . . . . . . . 15

2 Model Development . . . . . . . . . . . . . . . . . 16

2.1 Preliminary Work . . . . . . . . . . . . . . 16

2.1.1 Governing Equations . . . . . . . . . . . . . 17

2.1.2 Assumptions. . . . . . . . . . . . . . . . . . 18

2.1.3 Boundary Conditions . . . . . . . . . . . . . . 18

2.1.3.1 Wall ................. 19

2.1.3.2 Axis ..................... 22

2.1.3.3 Interior . . . . . . . . . . . . . 22

2.1.4 MATLAB Analytical Validation Models . . . . . . . . . . . . 22

2.1.4.1 Spin-up Meniscus Height . . . . . . . . . 23

2.1.4.2 Acceleration and Wall Shear Stress . . . . . . . 25

2.2 CFD-Finite Volume Method . . . . . . . . . . . . 26

2.2.1 Validation Model ................. 29

2.2.1.1 Setup ........................ 29

2.2.1.2 Analytical Solutions for Validation Model . . . . . . 32 
2.2.1.3 Mesh Convergence Studies . . . . . . . . . . . . 37

2.2.2 2-D Axisymmetric Model Studies . . . . . . . . . . . . . 41

2.2.3 3-D Model Development and Studies . . . . . . . . . . . . 44

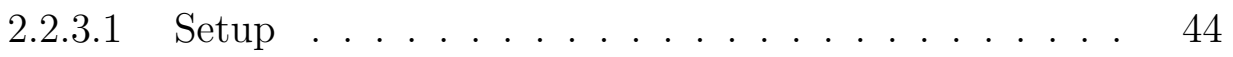

$2.2 .3 .2 \quad$ Studies . . . . . . . . . . . . . . 48

3 Testing \& Validation . . . . . . . . . . . . . . . . . . . . . . . 49

3.1 Experimental Setup . . . . . . . . . . . . . . . . . . . . . 49

4 Results \& Discussion . . . . . . . . . . . . . . . . . . . 55

$4.11 \mathrm{~mL}$ Syringe . . . . . . . . . . . . . . . . . . . . 55

4.1 .1 Results . . . . . . . . . . . . . . . . 55

$4.1 .2 \quad$ Discussion . . . . . . . . . . . . . . . . 61

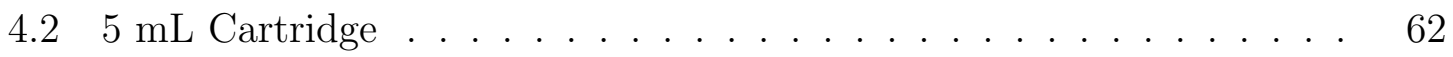

4.2 .1 Results . . . . . . . . . . . . . . . . . . . . . . 62

$4.2 .2 \quad$ Discussion . . . . . . . . . . . . . . . . . . 73

$4.32 .25 \mathrm{~mL}$ Syringe . . . . . . . . . . . . . . . . . . . . 73

4.3 .1 Results . . . . . . . . . . . . . . . . . . . . 73

$4.3 .2 \quad$ Discussion . . . . . . . . . . . . . . . . . . . . . 74

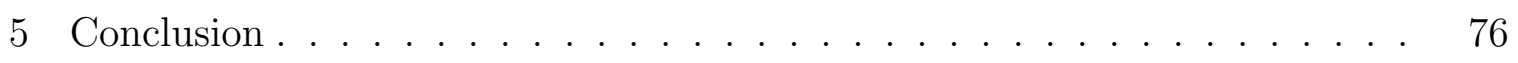

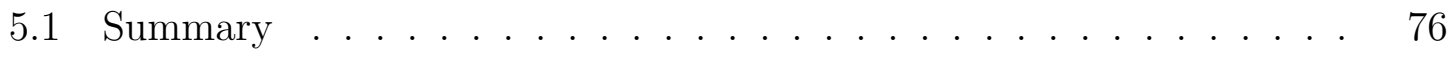

5.2 Future Work . . . . . . . . . . . . . . . . . . . . . 77

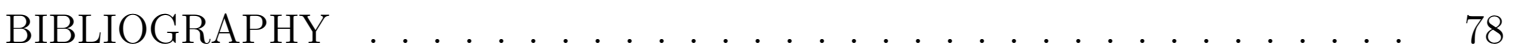
APPENDICES

A MATLAB Code .......................... 82

B Sample List for Experiment . . . . . . . . . . . . . . . . 91 


\section{LIST OF TABLES}

Table

Page

2.1 Solution method setup used in Fluent. . . . . . . . . . . . . . 27

2.2 Computational time difference between personal computer and Bishop cluster. . . . . . . . . . . . . . . . . . . . . 29

2.3 Validation Model Parameters . . . . . . . . . . . . . . 31

2.4 Mesh sizes with growth factors for the mesh convergence studies. . 37

2.5 Different viscosity values for $5 \mathrm{~mL}$ cartridge with an angular velocity of $3000 \mathrm{rpm}$. . . . . . . . . . . . . . . . . . . . . 43

2.6 Different surface tension values for a $4000 \mathrm{rpm} 2.25 \mathrm{~mL}$ syringe. . . 43

$2.7 \quad$ 3-D Simulation Cases for Fluent . . . . . . . . . . . . . . 48

4.1 Meniscus height of a $1 \mathrm{~mL}$ syringe for Fluent, MATLAB, and experiment. . . . . . . . . . . . . . . . 57

4.2 Meniscus Height of a $5 \mathrm{~mL}$ Cartridge at $3000 \mathrm{rpm}$ for Fluent and

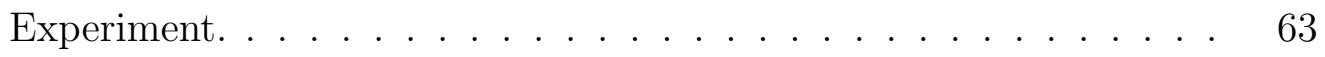

B.1 Sample list for experimental setup with air gap and viscosity values. $\quad 92$ 


\section{LIST OF FIGURES}

Figure

1.1 A diagram of a camera taking an image of a drug product container and an example of the captured image with the region of interest for particle detection. . . . . . . . . . . . . . . . . . . . . . .

1.2 A diagram of the spin-stop method for a $5 \mathrm{~mL}$ cartridge placed in the chuck. . . . . . . . . . . . . . . 3

1.3 Axial and cross-section of rotating cylinder in cylindrical coordinates. 6

1.4 Partially filled rotating liquid in a cylindrical tank showing a parabolic free surface. . . . . . . . . . . . . . . . . . . . . . . 9 9

1.5 For high $\omega$, an axial and cross section of a closed rotating tank. . . 10

2.1 A diagram of a $1 \mathrm{~mL}$ syringe spinning. . . . . . . . . . . . . 17

2.2 Comparison of net wall shear stresses for different start-up times. . 20

2.3 Density contour plots for different contact angles at $\mathrm{t}=2.5 \mathrm{~s}$. . . . 21

2.4 A comparison of net wall shear stress with different contact angles. 22

2.5 A diagram of a container at rest (a) and during a steady state spin (b) showing the meniscus height difference. . . . . . . . . . . . . . 23

2.6 A diagram of Lubarda's case to find the surface profile. . . . . . . . 24

2.7 An image of a mirrored 2-D axisymmetric mesh. . . . . . . . . . 30

2.8 A diagram comparison between the created 2-D mesh using Fluent's axisymmetric coordinate with the physical geometry. . . . . . . . . 30

2.9 The shape of the surface profile with corresponding Bond number, eta, and contact angle. . . . . . . . . . . . . . . 32

2.10 Analytical solutions of velocity profiles for an infinite cylinder with no velocity change. . . . . . . . . . . . . . . . .

2.11 Analytical solutions of velocity profiles for an infinite cylinder with velocity change at outer wall. . . . . . . . . . . . . . . . 34

2.12 Velocity profiles for the Fluent model at $1800 \mathrm{rpm}$. . . . . . . . . . 35

2.13 Average Wall shear stress for the Fluent model with analytical solution 36

2.14 Images of the four 2-D meshes for the mesh convergence studies. . 37

2.15 Density contour plot with approximate meniscus height in Fluent. . 38

2.16 A diagram of a MATLAB plot to determine the meniscus height. . 38 
2.17 Mesh convergence plot for density height across mesh nodes at $t=5$ seconds. . . . . . . . . . . . . . . . . . . . . . 39

2.18 Mesh convergence plot for net wall shear stress over time. . . . . 40

2.19 A plot of a mesh convergence closeup of Figure 2.18b. . . . . . . 41

2.20 A mesh for the $5 \mathrm{~mL}$ cartridge. . . . . . . . . . . . . . . 42

2.21 A mesh for the $2.25 \mathrm{~mL}$ syringe . . . . . . . . . . . . . 42

2.22 A Diagram of the solid bodies in a 3D mesh. . . . . . . . . . 44

2.23 3-D mesh for a $1.5 \mathrm{~mL}$ syringe and $5 \mathrm{~mL}$ cartridge. . . . . . . . . 46

2.24 Close up view for a $1 \mathrm{~mL}$ syringe and a $5 \mathrm{~mL}$ cartridge. . . . . . . 47

$3.1 \quad$ A Solidworks rendering of the SpinCAM . . . . . . . . 49

3.2 A front view of SpinCAM with labeled key parts. . . . . . . . . 50

3.3 A view of the spin CAM with the blue cover. . . . . . . . 50

3.4 A view of the SpinCAM showing a Basler camera with telephoto lens capturing images. . . . . . . . . . . . . . . . 51

3.5 A view of the camera facing the spinning cartridge with flashing LED lights. . . . . . . . . . . . . . . . . 51

$3.65 \mathrm{~mL}$ cartridge placed on the chuck inside the SpinCAM. . . . . . . 52

3.7 A view of the syringe chuck inside the SpinCAM. . . . . . . . . 53

3.8 A front view of the $1 \mathrm{~mL}$ syringe, $2.25 \mathrm{~mL}$ syringe, and $5 \mathrm{~mL}$ cartridge 54

3.9 An image of measuring the dimensions for the $5 \mathrm{~mL}$ cartridge . . . 54

4.1 An image of a $1800 \mathrm{rpm} 1 \mathrm{~mL}$ syringe at steady state with pixel dimensions in MATLAB. . . . . . . . . . . . . 56

4.23 -D models of a $1 \mathrm{~mL}$ syringe spinning at $1800 \mathrm{rpm}$ at 2.5 seconds. 56

$4.3 \quad$ Plots for a $1 \mathrm{~mL}$ filled $1 \mathrm{~mL}$ syringe at $5000 \mathrm{rpm}$. . . . . . . . 58

4.4 A streamline plot during steady state at 2.5 seconds for a $1 \mathrm{~mL}$ syringe spinning at $5000 \mathrm{rpm} . \ldots \ldots \ldots$

4.5 A sectional density contour plot for a $1 \mathrm{~mL}$ syringe spinning down to rest from 2.5 to 2.75 seconds. . . . . . . . . . . . . . . . 60

$4.65 \mathrm{~mL}$ cartridge spinning at $3000 \mathrm{rpm}$ using pixel measurements . . 62

4.7 Captured images from the SpinCAM for a $5 \mathrm{~mL}$ cartridge spinning at $3000 \mathrm{rpm} \ldots \ldots \ldots \ldots \ldots \ldots \ldots$

4.8 Density contour plots from Fluent for a $5 \mathrm{~mL}$ cartridge spinning at $3000 \mathrm{rpm}$ to rest. . . . . . . . . . . . . . . . 65 
4.9 Streamline contour plot from Fluent for a $5 \mathrm{~mL}$ cartridge spinning

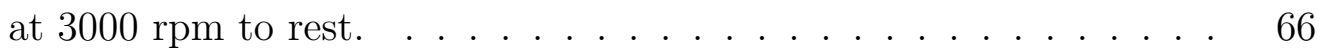

4.10 Wall shear stress for dynamic viscosity of 5, 10, and $20 \mathrm{cP} \ldots \ldots 67$

4.11 Average Angular Velocity for dynamic viscosity of 5, 10, and $20 \mathrm{cP} . \quad 68$

4.12 Density contour plot comparison at $\mathrm{t}=3.0$ seconds . . . . . . . . 69

4.13 A sectional density contour plot for a $5 \mathrm{~mL}$ cartridge spinning at $3000 \mathrm{rpm}$ at 1.5 seconds. . . . . . . . . . . . . . 70

4.14 A sectional density contour plot for a $5 \mathrm{~mL}$ cartridge spinning down to rest from 1.5 to 3 seconds. . . . . . . . . . . . . . . . . 71

4.15 Streamline comparison between the 2-D and 3-D Fluent model. . . 72

4.16 Surface tension comparison of a $2.25 \mathrm{~mL}$ syringe at a) $0.048 \mathrm{~N} / \mathrm{m}$, b) $0.072 \mathrm{~N} / \mathrm{m}$, and $0.096 \mathrm{~N} / \mathrm{m}$. . . . . . . . . . . . . 74 


\section{Chapter 1}

Introduction

\subsection{Objective}

The objective of this thesis is to provide 1) a better understanding of the fluid mechanics in biotechnology containers using Computational Fluid Dynamics (CFD) models, 2) study the cause of the bubble formation in traditional spin-up and spin-down cases, and 3) gain insight into future improvements in drug product quality.

\subsection{Motivation}

Amgen Inc. is a biotechnology company focused on creating innovative solutions in therapeutic medicine. They are notable for creating industry-leading drug products such as Aranesp, Enbrel, and Neupogen. These products undergo extensive quality inspection under the Food and Drug Administration (FDA) regulations. Amgen's Technology and Automation for Operations (TAO) is involved with supporting the existing commercial automated inspection systems in addition to developing proprietary technologies for Amgen's quality and manufacturing processes.

A critical component of the drug manufacturing process is the inspection process to ensure the quality and purity of drug-filled containers. The commercial automated inspection systems are used to detect undesired particles suspended in locations in the drug product such as the meniscus of the liquid and the container walls. The automated inspection systems cannot detect the particles in these locations because they remain hidden in 2-D images as shown in Figure 1.1 where a particle is undetected at the container side wall. Therefore, the drug products must undergo a form of 
agitation to drive the particles away from the meniscus, the surface profile between the gas and liquid interface, and the boundary walls to detect these unwanted particles.

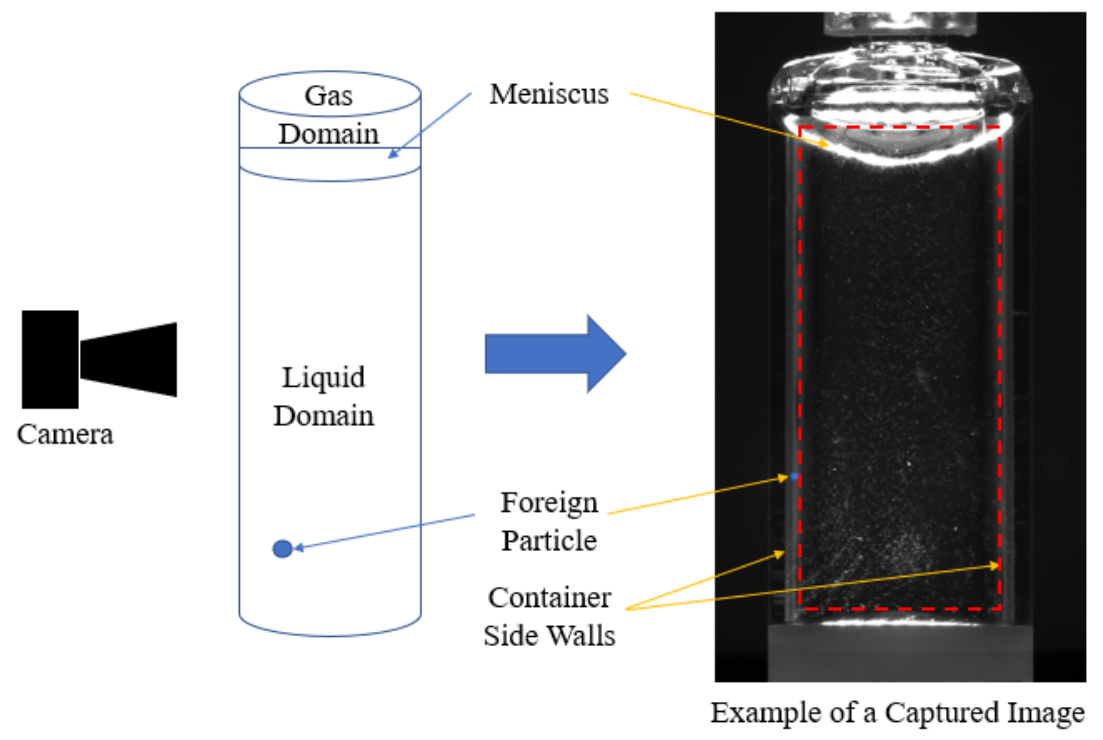

Figure 1.1 - A diagram of a camera taking an image of a drug product container and an example of the captured image with the region of interest for particle detection.

Amgen currently uses the spin-stop method to agitate the particles away from the walls. The containers spin-up to a certain angular velocity, $\omega$, and spins down to rest as shown in Figure 1.2. After a few milliseconds when the container is at rest, a high speed camera takes images of the particles swirling inside the container as shown in Figure 1.1. The images are taken afterwards to minimize the noise and vibrations of the container during the spin-stop process. These images are processed as binary images to locate any particles in the drug product containers and reject them. 


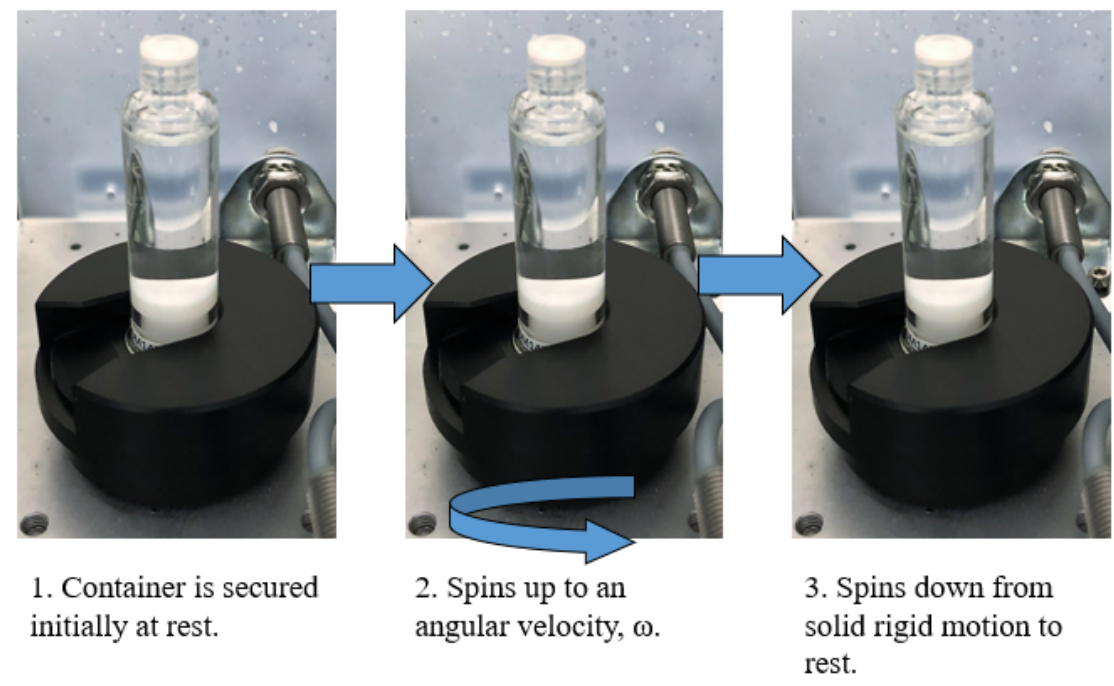

Figure 1.2 - A diagram of the spin-stop method for a $5 \mathrm{~mL}$ cartridge placed in the chuck.

Another particular of concern for drug product containers is bubble formations occurring during the spin-stop process. Bubble formations are known to cause damages to the integrity of the proteins from mechanical shocks from Randolph's studies [1], but it is undetermined whether these same bubbles are occurring during the spin-stop process. Due to the conservative FDA guidelines to ensure the proteins in the drug product are pure and safe, measures must be taken to avoid these bubble formations that can cause potential harmful effects to the proteins.

Therefore, the CFD models can provide a better understanding of the fluid movements and optimal agitation methods during the spin-stop process as a quantitative and visual tool to assess needs for improvements in quality and production. Process parameters such as viscosity, angular velocity, surface tension, contact angle, and fill volume will be studied to optimize the process. Also, the CFD models can further investigate the causes for bubble formations during the spin-stop process and how to possibly avoid it. These studies will be part of a larger goal for TAO's mission to investigate the spin-stop method's impact on the future of drug product quality. 


\subsection{Previous Work}

Studies of rotating fluids in cylinders originate about fifty years ago with research in spinning fluid tanks, centrifuges, and liquid filled projectiles from ballistic missiles $[2,3,4]$. Most recently, these studies have expanded to research in biological and

chemical purposes [5] as well as applications in centrifugal castings for mirrors and contact lenses [6].

Rotating fluid in cylinders extends to Amgen's use of the spin-stop method to rotate fluids in a container. The spin-stop process is comprised of a spin-up from rest and spin-down to rest to agitate the particles away from the meniscus and wall boundaries. Therefore, this section is organized in three subsections of 1) spin-up, 2) steady state meniscus shapes, and 3) spin-down. The focus for the subsections are the effects from the process parameters, instabilities, and bubble formations during the spin-stop process.

\subsubsection{Spin-up}

The research for impulsively started spin-up from rest in cylinders have been focused on the analytic, experimental, and numerical solutions $[7,8,9,10,11]$ for liquid filled and partially filled containers. Process parameters such as surface tension, viscosity, angular velocity, and fill volume were used in these studies.

In order to characterize the various process parameters, dimensionless numbers are used to determine their significance. The Reynolds number, Ekman number, and aspect ratio for any fluid container are important dimensionless numbers for both partial and filled liquid containers, which are shown as

$$
R e=\frac{\omega a^{2}}{\nu}
$$




$$
\begin{gathered}
E=\frac{\nu}{\omega h^{2}}, \\
A=\frac{h}{a},
\end{gathered}
$$

where $a$ is the radius of the fluid, $h$ is the height of the fluid, $\nu$ is the kinematic viscosity, and $\omega$ is the angular velocity.

Equation 1.1 describes the ratio of inertial forces over viscous forces to determine whether the fluid flow is laminar or turbulent. From Schlicting [12], the critical Reynolds number for a rotating disk to remain laminar is $R e<300,000$. This critical Reynolds number was further experimentally verified by Choi [13], Shadday [14], and Yan [5] for the laminar cases of rotating containers.

For Equation 1.2, the Ekman number describes the ratio of viscous forces to Coriolis forces, and typically determines the planetary rotation effects on oceans. However, when $E<<1$ and used for containers, it assumes the viscous effects are limited to the boundary layers near the walls, and further demonstrates the meridional circulation by the Ekman suction at the end walls [9]. The aspect ratio was shown in Equation 1.3 of a dimensionless ratio for the geometry of the container. 


\subsubsection{Liquid Filled Container}

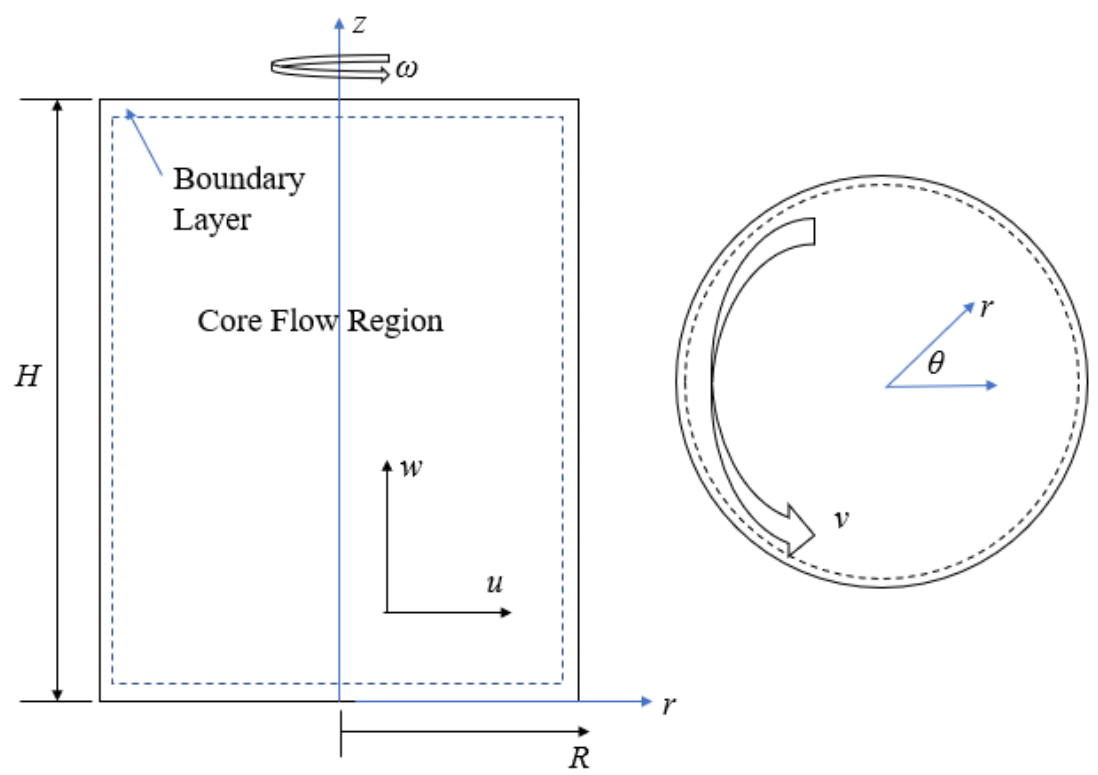

Figure 1.3 - Axial and cross-section of rotating cylinder in cylindrical coordinates.

For liquid filled containers, Wedemeyer [7] used the Navier-Stokes and continuity equations to describe a fluid-filled spinning cylinder of finite length spinning about its axis. Wedemeyer [7] investigated a liquid filled spinning shell and found his analytical solutions to show a good agreement with his experimental observations. Ibrahim [2] showed in Figure 1.3 that the axial and cross-section of the spinning cylinder in cylindrical coordinate frame, $r, \theta$, and $z$, with velocity components, $u, v$, and $w$, are shown as

$$
\begin{gathered}
\frac{\partial(r u)}{\partial r}+\frac{\partial(r w)}{\partial z}=0 \\
\frac{\partial u}{\partial t}+u \frac{\partial u}{\partial r}+w \frac{\partial u}{\partial z}-\frac{v^{2}}{r}+\frac{1}{\rho} \frac{\partial p}{\partial r}=\nu\left[\frac{\partial^{2} u}{\partial r^{2}}+\frac{\partial}{\partial r}\left(\frac{u}{r}\right)+\frac{\partial^{2} u}{\partial z^{2}}\right], \\
\frac{\partial v}{\partial t}+u\left(\frac{\partial v}{\partial r}+\frac{v}{r}\right)+w \frac{\partial v}{\partial z}=\nu\left[\frac{\partial^{2} v}{\partial r^{2}}+\frac{\partial}{\partial r}\left(\frac{v}{r}\right)+\frac{\partial^{2} v}{\partial z^{2}}\right]
\end{gathered}
$$




$$
\frac{\partial w}{\partial t}+u \frac{\partial w}{\partial r}+w \frac{\partial w}{\partial z}+\frac{1}{\rho} \frac{\partial p}{\partial z}=\nu\left[\frac{\partial^{2} w}{\partial r^{2}}+\left(\frac{1}{r}\right) \frac{\partial w}{\partial r}+\frac{\partial^{2} w}{\partial z^{2}}\right]
$$

The boundary conditions for this case are $u=w=0, v=\Omega r$ at $z=0$ and $h$ and $u=w=0, v=R \Omega$ at $r=R$ with $u=v=0$ at $r=0$. Wedemeyer[7] divided the flow region as the boundary-layer region near the end walls, and the core flow as the remaining flow. Equations 1.4, 1.5, 1.6, and 1.7 represent the boundary layer region near the end walls to obtain the analytical solutions. The Navier-Stokes and continuity equations for core flow are

$$
\begin{gathered}
\frac{1}{\rho} \frac{\partial \rho_{0}}{\partial r}=\frac{v_{0}^{2}}{r} \\
\frac{\partial v_{0}}{\partial t}+u_{0}\left(\frac{\partial v_{0}}{\partial r}+\frac{v_{0}}{r}\right)=\nu\left[\frac{\partial^{2} v_{0}}{\partial r^{2}}+\frac{\partial}{\partial r}\left(\frac{v_{0}}{r}\right)\right],
\end{gathered}
$$

where $u_{0}, v_{0}$, and $p_{0}$ are the velocity and pressure components for the core flow. The core flow described in Equation 1.8 and 1.9 are often described by other researchers as the interior flow $[2,3]$. The boundary layer flow is treated as quasi-steady; therefore, the local acceleration terms are ignored and the boundary layer equations become

$$
\begin{gathered}
\frac{\partial(r u)}{\partial r}+\frac{\partial(r w)}{\partial z}=0 \\
u \frac{\partial u}{\partial r}+w \frac{\partial u}{\partial z}-\frac{v^{2}}{r}+\frac{v_{0}^{2}}{r}=\nu \frac{\partial^{2} u}{\partial z^{2}} \\
u\left(\frac{\partial v}{\partial r}+\frac{v}{r}\right)+w \frac{\partial v}{\partial z}=\nu \frac{\partial^{2} v}{\partial z^{2}} .
\end{gathered}
$$

According to Greenspan [3], a cylinder with filled liquid speeds up from rest to the desired angular velocity, $\omega$, and reaches solid rigid body motion after a few seconds. Throughout the transient process, the surface profile changes creating a vortex at the 
axis of the spinning cylinder. Greenspan [3] found there are three phases during spinup; the Ekman boundary layer, the secondary flow, and the viscous decay of residual modal oscillations. In Greenspan's experiment, a closed axisymmetric container of disks filled with viscous incompressible fluid rotates with a constant angular velocity about a fixed axis with an incremental change in velocity [3]. The spin-up time to reach steady state is shown as

$$
T=\left(L^{2} / \nu \Omega\right)^{\frac{1}{2}}
$$

where $L$ is the characteristic length that is parallel to the axis of container, $\nu$ is the kinematic viscosity, $\Omega$ is the angular velocity, and $T$ is the spin-up time to reach rigid body motion. During spin-up, the Coriolis force outweigh the pressure gradient and form a source near the bottom of the cylinder, causing the flow to radially move outwards [3]. The Ekman layer eventually decays when the rotating cylinder approaches steady state and the viscous forces become dominant in the interior flow to get rid of any modal oscillations [3]. Greenspan [3] found that Equation 1.13 agreed well with his observations and experiments for the transient motion in the interior flow. Greenspan [3] also compared the lubricating oil at $1000 \mathrm{cP}$, water at $1 \mathrm{cP}$, and Mercury at $0.1 \mathrm{cP}$ for Reynolds numbers of $1 \mathrm{E} 10^{5}, 1 \mathrm{E} 10^{6}$, and $1 \mathrm{E} 10^{7}$, respectively. Using Equation 1.13, the time to reach steady state was faster with a higher viscosity and lower Reynolds number.

Watkins and Hussey [15] used a kinematic viscosity range of 0.0088 to $0.34 \mathrm{~cm}^{2} / \mathrm{s}$ for a closed cylinder spinning up from rest. Reynolds number, Ekman number, and aspect ratio ranging from $1.6 \mathrm{E} 10^{4}$ to $2.55 \mathrm{E} 10^{5}, 3.44 \mathrm{E} 10^{-6}$ to $1.56 \mathrm{E} 10^{-5}$ and 0.280 to 2.0, respectively, were tested numerically and experimentally for velocity profiles with previous researcher's models. Watkins and Hussey [15] found that comparing different geometric and fluid parameters can be done when using the same value for the following dimensionless parameter, 


$$
\alpha_{0}=h(\nu / \omega)^{1 / 2} / a^{2}=A^{-2} E^{1 / 2} .
$$

where $A$ and $E$ are the aspect ratio and Ekman number, respectively. Watkins and Hussey [15] concluded that when Equation 1.14 was less than 0.005 , the velocity profiles agreed well with Wedemeyer's model[7].

\subsubsection{Partially-Filled Liquid Container}

There have been studies by researchers regarding partially filled liquid in open and closed containers. Ibrahim [2] noted that researcher Miles and Ball $[16,17]$ studied the free-oscillations in a rotating paraboloid. Figure 1.4 shows the rotating paraboloid with the meniscus behavior at the free surface with the equation to solve for the

profile at $y=\frac{\Omega^{2}}{2 g} x^{2}$ by Ibrahim [2]. The surface profile equation shows a relationship between the angular velocity and the gravitational acceleration with the meniscus height.

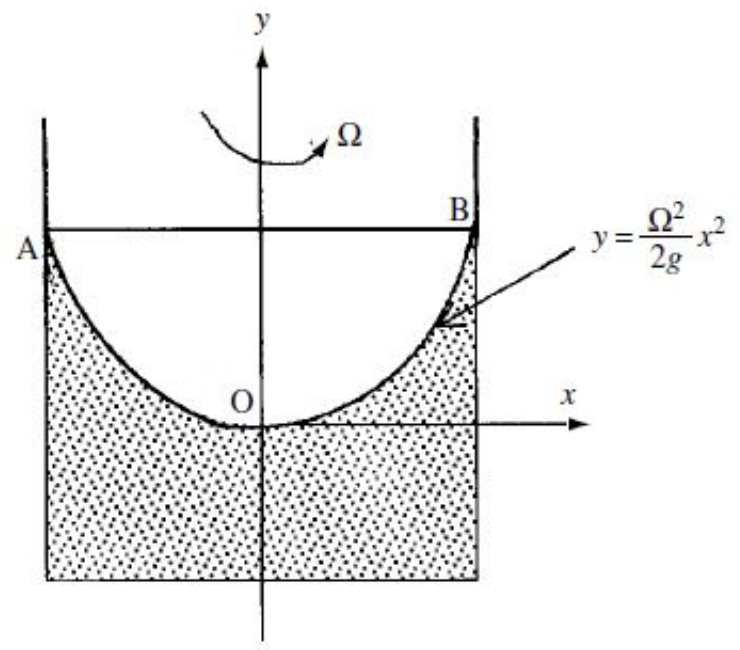

Figure 1.4 - Partially filled rotating liquid in a cylindrical tank showing a parabolic free surface. 


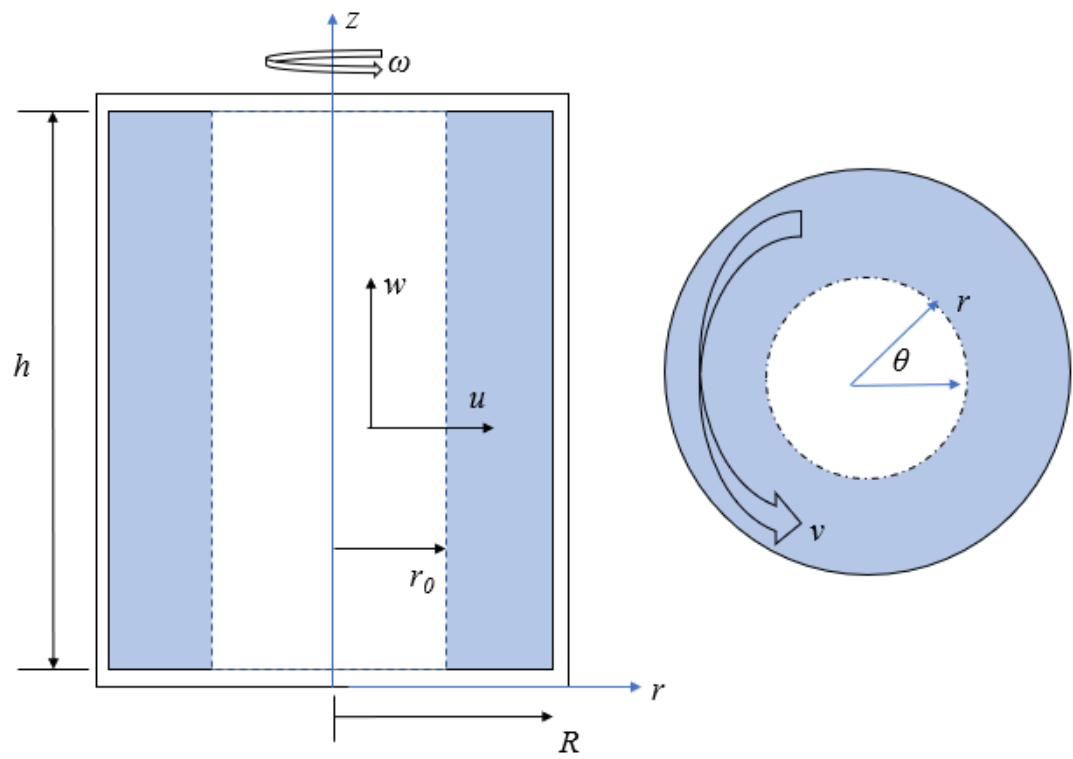

Figure 1.5 - For high $\omega$, an axial and cross section of a closed rotating tank.

For a closed cylinder where liquid cannot spill out of the container, Miles and Troesch [18] investigated an annular ring forms when the angular velocity increase. Ibrahim [2] showed in Figure 1.5 that when a closed tank is rotating, the liquid rises and forms an annular ring where the shaded region is liquid and the white region is air. Using the coordinate system in Figure 1.5, Miles and Troesch $[2,18]$ formed equations for this case as shown as

$$
\begin{gathered}
\frac{\partial u}{\partial t}-2 \omega v \omega^{2} r=-\frac{1}{\rho} \frac{\partial P}{\partial r}, \\
\frac{\partial v}{\partial t}+2 \omega u=-\frac{1}{\rho r} \frac{\partial P}{\partial \theta}, \\
\frac{\partial(u r)}{\partial r}+\frac{\partial v}{\partial \theta}=0, \\
P=\frac{\rho \omega^{2}}{2}\left(r^{2}-r_{0}^{2}\right)+p(r, \theta, t),
\end{gathered}
$$


where $\omega$ is the angular velocity, $P$ is the total pressure, $p(r, \theta, t)$ is the pressure due to small movements of the liquid, and $r_{0}$ is the radius of the inner ring of air. Equations 1.15 and 1.16 are derived from the Navier-Stokes equations, while Equation 1.17 is the continuity equation. By solving Equations 1.15, 1.16, 1.18 and substituting into Equation 1.17, a differential equation formed as given by

$$
r^{2} \frac{\partial^{2} p(r)}{\partial r^{2}}+r \frac{\partial p(r)}{\partial r}-n^{2} p(r)=0 .
$$

Equation 1.19 created a boundary value problem for oscillations during the twodimensional spin-up case by Miles and Troesch [18].

Gerber [19] qualitatively investigated two partially filled containers with a dimensionless fill ratio of $b / h=0.2$ and $b / h=0.8$ where $b$ is the height of the liquid and $h$ is the container height. The case for $b / h=0.2$ described a case when the container was less than half-filled and created an annular ring. In contrast, a fill ratio of $b / h=0.8$ was more than half-filled, which showed a paraboloid shaped annular ring. According to Gerber[19], once the surface profile reached the bottom endwall, the growth rate of the meniscus reduced, indicating the diminishing involvement of the Ekman layer. Based on Gerber's[19] resarch, angular velocity and fill volume parameters played an important role in the meniscus height and shape as well as the time to reach steady state.

Homicz and Gerber [20] performed a numerical model scheme using the CrankNicolson finite difference scheme on an uniform grid, in which they described the three sequential stages of the meniscus becoming parabolic and stretching to the bottom until it reached a steady state. Homicz and Gerber [20] used Reynolds numbers of $1.172 \mathrm{e} 10^{5}, 1.172 \mathrm{e} 10^{4}$, and $1.172 \mathrm{e} 10^{3}$, which showed a diminishing reduction in the spin-up rate when the Reynolds number decreased. Choi and Kim [13] confirmed Hommicz and Gerber's analytical model with experimental results. 
Yan and Farouk [5] investigated an eighty percent filled container with water using a homogeneous multiphase model and a control volume finite difference numerical scheme to solve the Navier-Stokes equations for a partially filled centrifuge. The partially filled container had dimensionless numbes of $A=3.0$ and $R e=1.172 \mathrm{e} 10^{5}$, and $\mathrm{E}=9 \cdot 48 \mathrm{e} 10^{-7}[5]$. According to Yan and Farouk [5], the suction mechanism from the Ekman boundary layers and the radially inward circulations formed on the endwalls and interior flow, respectively. Surface tension effects on the wall were neglected in these studies due to the large container diameters of $94 \mathrm{~mm}$ and a height of $141 \mathrm{~mm}$.

The numerical simulations were performed on a IBM-RISC-6000 workstation with computation time ranging from 70 to 80 hours for each case [5]. Yan and Farouk [5] made sure to use a high number of nodes at the top and bottom of the surface profile. The calculations were done using the SIMPLE algorithm, QUICK differencing scheme, and an implicit differencing scheme for the time derivative. At a speed of $400 \mathrm{rpm}$, Yan and Farouk [5] found that the Ekman layer was a radial flow moving outward, which induced a secondary flow in the interior core region as the secondary flows decayed when it approached solid body rotation for both gas and liquid phase [5].

\subsubsection{Steady State Meniscus}

The spin-stop process uses high angular velocities to stretch the meniscus into a vortex at the axis of the containers to disrupt the liquid domain into moving the particles away from the boundary walls. This section describes the meniscus when the liquid filled container reaches steady state.

Lubarda [6] investigated the surface tension and contact angle playing a role in spin-up studies for an uniformly rotating cylinder, in which the shape of the liquid surface is stable for a given angular momentum. For high angular velocities, the liquid 
surface dewets at the bottom of the cylinder [6]. Lubarda [6] used the governing conservation of angular momentum equations as follows:

$$
\begin{gathered}
\kappa(r)=\kappa(0)+\frac{\gamma_{l}}{2 \sigma_{l v}}\left[z(r)-z(0)-\frac{\omega^{2}}{2 g} r^{2}\right], \\
\cos \theta=\frac{\sigma_{s v}-\sigma_{s l}}{\sigma_{l v}} .
\end{gathered}
$$

Equation 1.20 is the differential equation representing the shape of the liquid and vapor interface where $k(0)$ is the bottom of the meniscus curvature, $\gamma_{1}$ is the specific weight of the liquid, $\sigma_{l v}$ is the liquid/vapor surface energy, $z(r)$ is a function of the liquid height with respect to the radius, $z(0)$ is the center of the container or the axial location for a liquid height, $\omega$ is the angular velocity, $g$ is the gravity, and $r$ is the radius. Equation 1.21 refers to the Young's equation for contact angle where $\theta$ is the contact angle, $\sigma_{s v}$ is the solid/vapor surface energy, $\sigma_{s l}$ is the solid/liquid surface energy, and the $\sigma_{l v}$ is the liquid/vapor surface energy. These equations define the shape of the meniscus profile during steady state. However, it does not take into account for the scenario during an impulsively started spin-up from rest. Lubarda [6] found that the contact angle was independent of the gravity and angular velocity for the differential equations.

\subsubsection{Spin-down to Rest}

The spin-down to rest cases have been studied by various researchers using experiments and numerical models to explain the instabilities in the fluid. It is known that instabilities and bubble formations can occur during the impulsive spin-down to rest $[21,22,23]$, which will be further explored in this section. 


\subsubsection{Instabilities and Effects of Taylor-Gortler Vortices}

During spin-down to rest, the wall shear stress caused instability in the flow. Along the wall, there are TG vortices forming during the instability. These studies were initially started by Euteneuer [24], who investigated experimentally the vorticies in the boundary layer along the concave walls called Taylor-Görtler (TG) vortices.

According to Neitzel and Davis [21], instabilities appeared from the sidewalls when a container filled with incompressible fluid was impulsively spun down to rest. They performed finite difference schemes to numerically solve the axisymmetric NavierStokes equations and used aspect ratios up to 3.0 and Reynolds numbers up to 4000 for their studies [21]. The computations revealed that the angular momentum and onset times of instabilities agreed well with Euteneuer's [24] observations as well as finding TG vortices present in all cases studies except when the aspect ratio and Reynolds number were $A=1.0$ and $R e=1000$, respectively. Furthermore, Neitzel and Davis $[22,21]$ used the energy method and numerical simulations to find the instability analysis regarding the TG vortices.

Further numerical simulations were done by Cui [25], who introduced a stability coefficient known as $S_{c}$ to relate the TG vorticies to the events of centrifugal force and the radial pressure gradient in the sidewall boundary layer. The integrated form of $S_{c}$ was the sidewall stability coefficient, $S_{b l c}$, which found that a local increase in the $S_{b l c}$ suggest a presence of the TG vortices as well as becoming a reliable tool in predicting TG vorticies [25].

According to Kim and Choi [26], the propagation theory yielded agreeable results between the instability criteria and the experimental observation at a Reynolds critical number, $R e_{c}=320$. Kim and Choi [26] compared with Mathis and Neitzel's [27] models on critical Reynolds numbers and experimental values, which showed a similar trend but a disagreement with the predicted Reynolds critical numbers. Mathis and 
Neitzel [27] had a Reynolds critical number of $R e_{c}=161$ while Kim and Choi [26] had 320 .

\subsubsection{Bubble Formation during Spin-Down}

There were studies that investigated the bubble formations and instabilities during spin-down by Watson and Neitzel [23]. When a cylinder container impulsively spun down to rest from a solid rigid motion, a bubble either generated or separated from the inner core flow at high angular velocities. Studies have called this phenomena as vortex breakdown [23, 28], in which the structure of the vortex undergoes sudden changes.

Bhattacharyya [28] concluded that as the spin-down time progressed, the separation bubble reduced in size and moved toward the upper stationary endwall. He found that the bubble radius reduced in size as spin-down time reached steady state for $R e=1300$ and the azimuthal component of the velocity, $\zeta$, had an important role in the vortex breakdown. In particular, the inertial rebound between the pressure gradient and the Coriolis forces caused a negative component of the azimuthal velocity [23]. 


\section{Chapter 2}

\section{Model Development}

This chapter is an overview of the preliminary work for the Fluent model, the twodimensional (2-D) case, the validation models, and the three-dimensional (3-D) case. The initial goals of the Fluent model will show an agreement with an analytic solution for a steady state meniscus shape conveyed in MATLAB as well as a mesh convergence study to show grid independence. Once these conditions are met, the simulation models of the different process parameters are conducted to show an agreement with experiments conducted at Amgen.

\subsection{Preliminary Work}

This section provides an outline of the governing physics, assumptions made on the model, and the imposed boundary conditions as referenced from Fluent's theory guide [29]. A diagram of the 2-D model for a $1 \mathrm{~mL}$ glass syringe spinning from the bottom is shown in Figure 2.1. The shaded region in blue is the mesh zone of interest for the two phase flow of air and water as shown in Figure 2.1. The bottom white portion of the syringe is not included in the mesh zone because it is typically plugged with a plunger, and therefore not bounded in the liquid mesh zone. Figure 2.1 shows the syringe spins about the vertical axis from the bottom, which is attached to a chuck connected to a motor. 


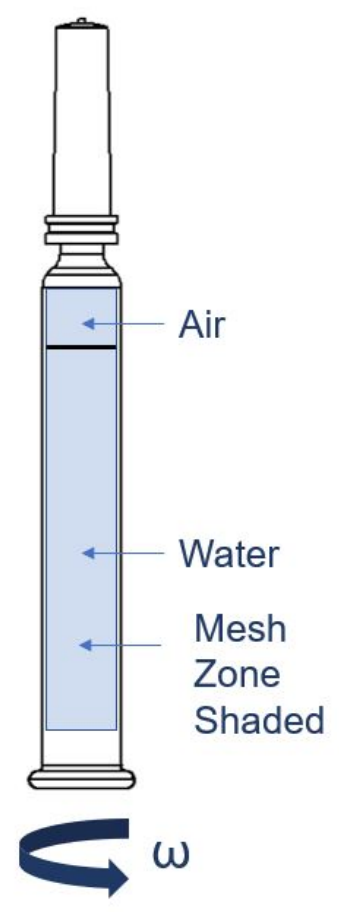

Figure 2.1 - A diagram of a $1 \mathrm{~mL}$ syringe spinning.

\subsubsection{Governing Equations}

The equations used in Fluent are the continuity and momentum equations. The most general form of equation for the continuity and momentum equation are

$$
\begin{gathered}
\frac{\partial \rho}{\partial t}+\nabla \cdot(\rho \vec{V})=0 \\
\frac{\partial}{\partial t}(\rho \vec{V})+\nabla \cdot(\rho \vec{V} \vec{V})=-\nabla p+\nabla \cdot\left[\nu\left(\nabla \vec{v}+\nabla \overrightarrow{V^{T}}\right)\right]+\rho \vec{g}+\vec{F},
\end{gathered}
$$

where $\rho$ is the density, $\vec{V}$ is the velocity tensor, $\rho \vec{g}$ is the gravitational body force, $\vec{F}$ is the external body force, $\nu$ is the molecular viscosity, and $p$ is the static pressure. $[29]$. 


\subsubsection{Assumptions}

There are several general assumptions for the Fluent model. These assumptions affect the results of our model as well as the computational time.

1. The density for the gas and liquid properties are at a room temperature of $25^{\circ}$.

2. Container is initially filled with air at the top and liquid at the bottom.

3. The cap for the container is assumed as a wall and not an outlet. Air is present in the cap, which is not accounted for in the model.

4. Surface tension and contact angle are constant properties through time.

5. Initially, $\omega$ increases linearly for $2.5 \mathrm{~ms}$ to desired $\omega$.

6. No initial air bubbles or any fibers are present inside the container.

7. The boundary conditions are uniformly distributed.

8. Laminar flow during the spin-stop process.

9. Swirl or circumferential velocity is present in the rotating fluid.

10. No-slip condition at the boundaries.

11. The container is geometrically axisymmetric about its central axis only for 2-D cases, and not assumed for 3-D cases.

\subsubsection{Boundary Conditions}

The boundary conditions for the spin-stop of a partially filled liquid cylinder for the 2-D axisymmetric using a $r, \theta$, and $z$ coordinate system with velocity components $V_{r}, V_{\theta}$, and $V_{z}$ are shown as:

1. $V_{\theta}=V_{z}=0, V_{r}=R \omega$ at $r=R$

2. $V_{\theta}=0, \frac{\partial V_{r}}{\partial r}=\frac{\partial V_{z}}{\partial r}=0$ at $r=0$

3. $V_{\theta}=V_{z}=0, V_{r}=r \Omega$ at $z=0$ and $z=H$ 
For a 3-D model, the boundary conditions using a $x, y$, and $z$ coordinate system with velocity components $u, v$, and $w$ are shown as:

1. $u=w=0, v=R \omega$ at $y=R$

2. $u=0, \frac{\partial v}{\partial r}=\frac{\partial w}{\partial r}=0$ at $y=0$

3. $u=w=0, v=r \omega$ at $z=0$ and $z=H$

\subsubsection{Wall}

The wall rotates relative to the adjacent cell zone at a specified angular velocity with a wall adhesion to describe the contact angle at the wall between the liquid and gas zones.

Initial study models were done using a constant angular velocity, $\omega$, to ensure the meniscus stretches downward as described from previous work [6, 19, 20]. The initial study models did not show any meniscus movement at high speeds of $5000 \mathrm{rpm}$ or higher with the constant angular velocity parameter in Fluent.

Therefore, the angular velocity needed a specified ramp up time for the desired speed, $\omega$, by using a profile file in Fluent where the angular velocity increases linearly for a desired time. A study was conducted to further understand the sensitivity of the start up times during spin-up. Figure 2.2 indicates the maximum net wall shear stress have different values where the net wall shear stress is highest at 2.85 pascals at $t=0.025$ seconds and the lowest at 2.40 pascals at $t=0.0005$ seconds. Although the maximum wall shear stresses are different, all values for different spin-up times converge and reach steady state at the same time. As a result, Figure 2.2 concludes there is a lack of sensitivity to different start-up times for the spin-up cases, and a start-up time of 2.5 millisecond was chosen as a reasonable assumption for all models. 


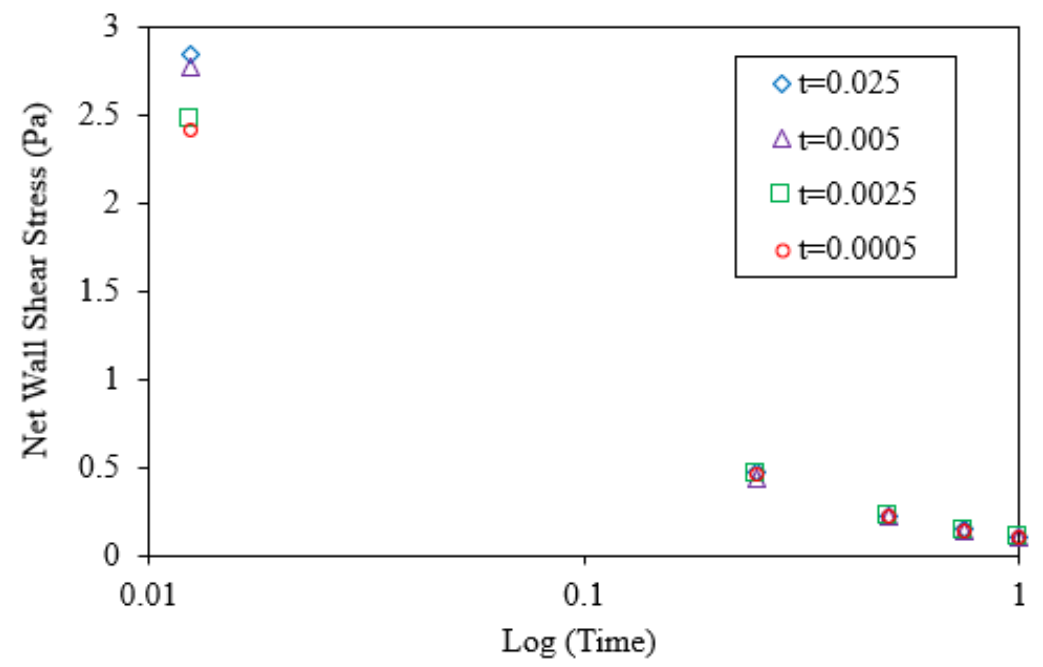

Figure 2.2 - Comparison of net wall shear stresses for different start-up times.

The contact angle at the wall was assumed as 45 degrees. To ensure the model was not sensitive to contact angles, a study using the validation parameters was done to see any noticeable impact with contact angles of 30, 45, and 60 degrees. During spin-up, the density contour plots were compared at $t=2.5$ seconds for the different contact angle values in Figure 2.3. The contact angles of 30, 45, and 60 are shown as Figure 2.3a, b, and c, respectively. By inspection, the meniscus height decreases as the contact angle increases. 

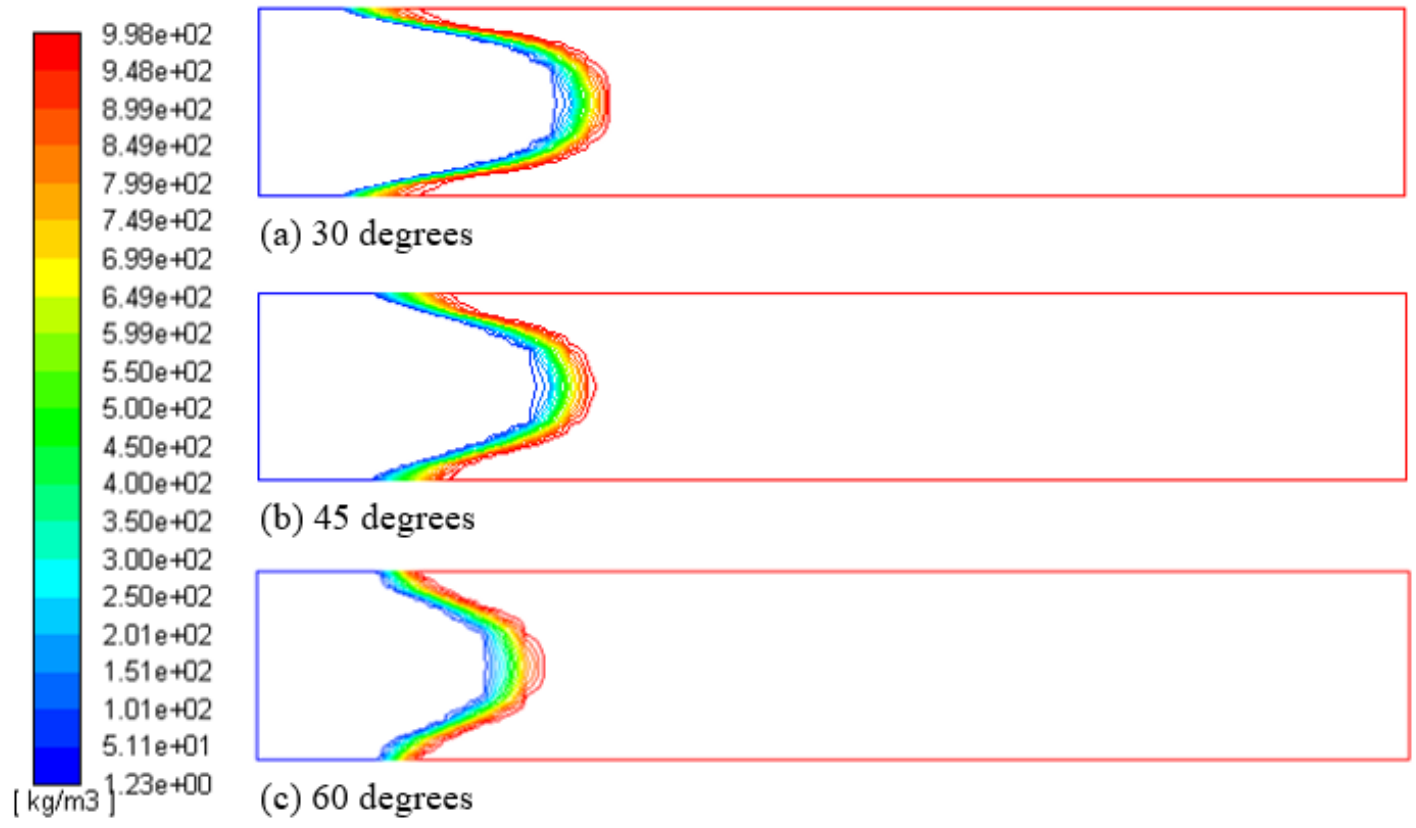

(a) 30 degrees

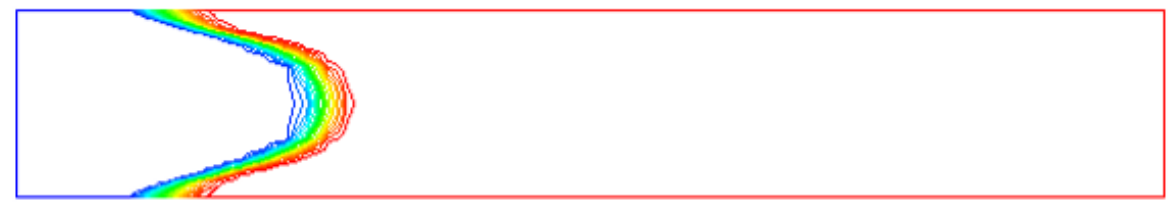

(b) 45 degrees

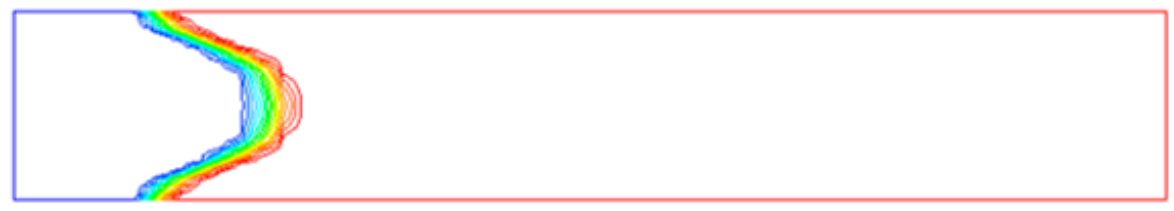

(c) 60 degrees

Figure 2.3 - Density contour plots for different contact angles at $\mathrm{t}=2.5 \mathrm{~s}$

To take a closer look at these differences, the net wall shear stresses were compared with the different contact angles in Figure 2.4. The differences in the magnitude of the net wall shear stress between a contact angle of 30 and 45 degrees do not indicate a sensitivity to the changes in the contact angle. However, a noticeable difference can be seen between 45 and 60 degrees as the net wall shear stress is greater by 0.5 pascals. The increase in the net wall shear stress indicates that the meniscus height decreases and reaches steady state at a slower time. Therefore, the meniscus height is sensitive to changes in the contact angles. For purposes of time and efficiency in producing simulation models, the contact angle was assumed as 45 degrees for all models in this research. 


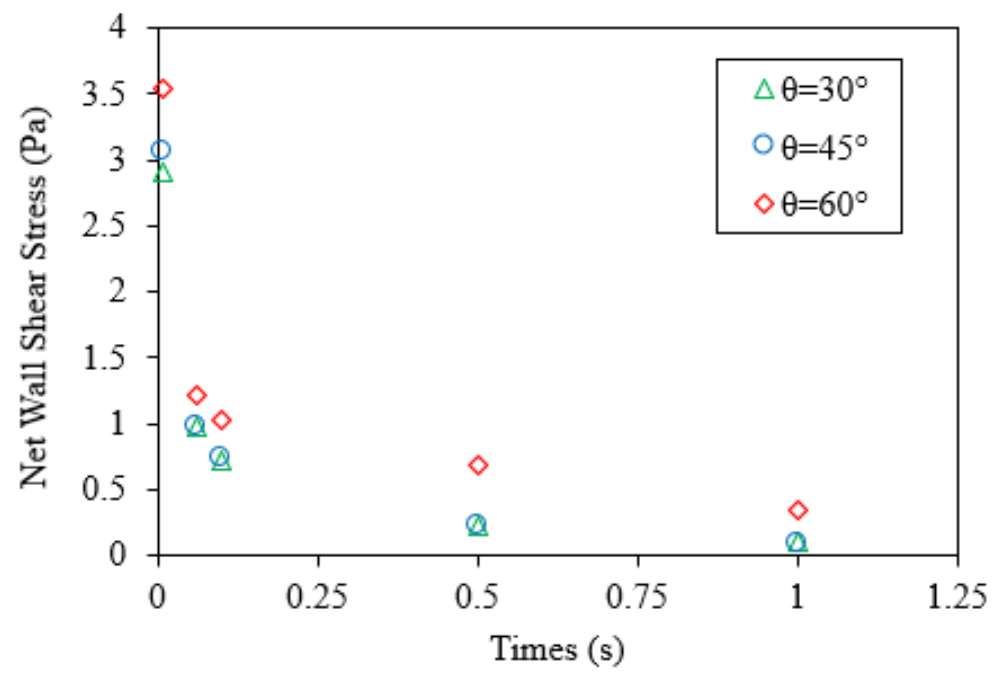

Figure 2.4 - A comparison of net wall shear stress with different contact angles.

\subsubsection{Axis}

The axis creates an axisymmetric boundary at the axis for the 2-D cases. This boundary condition will not be used for the 3-D model since it will not be axisymmetric.

\subsubsection{Interior}

The interior boundary conditions imposes a non-finite boundary between two different cell zones or flow properties. This is used to distinguish the profile between the liquid and gas zones.

\subsubsection{MATLAB Analytical Validation Models}

Analytical models are necessary to show the CFD models are valid. MATLAB scripts are created to validate the CFD models for finding analytical solutions for the meniscus height during spin-up as well as comparing the spin-up acceleration and wall shear stress. 


\subsubsection{Spin-up Meniscus Height}

A MATLAB script calculates the analytical solution of a meniscus height for a liquid filled cylinder at steady state using Lubarda's work [6]. Figure 2.5 shows a diagram of the spin-up case for a partially filled liquid with no a free top surface. Figure 2.5a shows the container with initial spin-up angular velocity, $\omega_{o}$, before reaching the desired angular showing the surface profile rising. Figure $2.5 \mathrm{~b}$ shows the container at solid rigid rotation without any viscous oscillations. This also does not include the meniscus height for a spin-down to rest. The script is constrained to an iterative solution based on input parameters where the meniscus height cannot exceed the container height, $H$, as shown in Figure 2.5 by Lubarda [6].
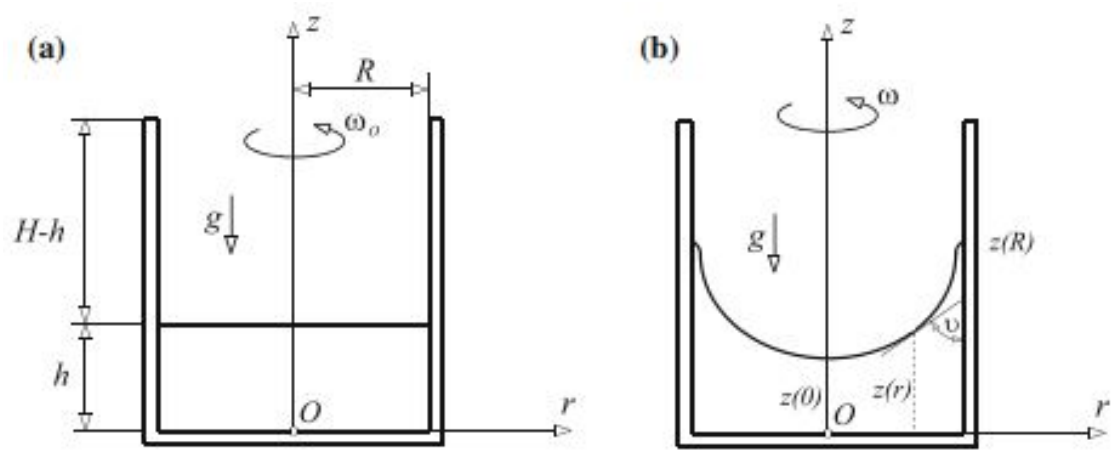

Figure 2.5 - A diagram of a container at rest (a) and during a steady state spin (b) showing the meniscus height difference. 


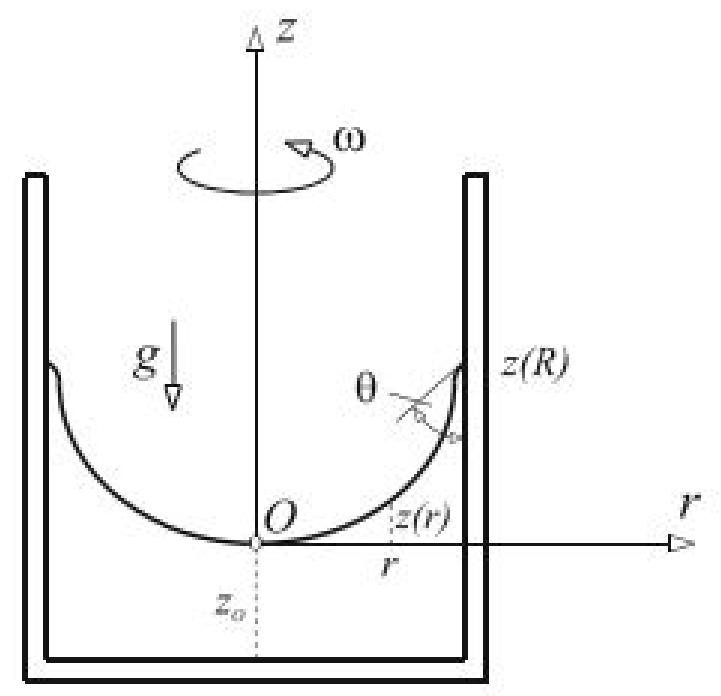

Figure 2.6 - A diagram of Lubarda's case to find the surface profile.

Figure 2.6 by Lubarda [6] shows a diagram of the reference configuration used to find the governing equations to find the surface profile height. The dimensionless numbers of interest are

$$
\begin{gathered}
B_{o}=\left(\frac{R}{l_{0}}\right)^{2}=\frac{R^{2} \rho_{l} g}{\sigma_{l v}}, \\
\eta=\frac{R \omega^{2}}{g},
\end{gathered}
$$

where $\omega$ is the angular velocity, $\sigma_{l v}$ is the surface tension of liquid-vapor interaction, $R$ is the radius of the interior container, $\rho_{l}$ is the liquid density, and $l_{o}$ is the capillary length, which is shown to be the squared root ratio of $\sigma_{l v}$ and $\rho_{l} g$. The dimensionless numbers from Equation 2.3 and 2.4 are used to form a non-dimensional differential equation from Lubarda [6],

$$
\frac{\bar{z}^{\prime \prime}}{\left(1+\bar{z}^{\prime 2}\right)^{3 / 2}}+\frac{\bar{z}^{\prime}}{\bar{r}\left(1+\bar{z}^{\prime 2}\right)^{1 / 2}}-B_{o} \bar{z}=2 \cos \theta-B_{o}\left[\bar{h}-\frac{\eta}{4}\left(1-2 \bar{r}^{2}\right)\right]
$$


where $\bar{r}=r / R, \bar{z}=z / R$, and $\bar{h}=h / R$. Equation 2.5 is numerically integrated in an iterative procedure in MATLAB to find the surface profile height at steady state. The MATLAB script is included in the appendices.

\subsubsection{Acceleration and Wall Shear Stress}

For an infinite fluid filled cylinder spinning up, the ramp time for velocity change is compared. This study calculates the wall shear stress change over a dimensionless time as well as the velocity profile over a dimensionless radius of the container. We used the dimensionless parameters such as Reynolds number, $R e$, and other numbers as shown

$$
\begin{gathered}
F r=\omega \sqrt{\frac{r}{g}}, \\
T_{o}=\frac{\nu t_{0}}{r^{2}},
\end{gathered}
$$

where $\omega$ is the angular velocity, $r$ is the radius, $\nu$ is the kinematic viscosity, $g$ is the gravitational acceleration, and $t_{0}$ is the velocity change or ramp up time. Equation 2.6 and 2.7 are the Froude number and dimensionless time to calculate the velocity profile and the shear stress. The Reynolds and Froude numbers are used to find the maximum wall friction during spin-up time. Equation 2.7 is used to compare the difference between velocity changes during the spin up time.

A MATLAB script was used to solve the velocity profile and wall shear stress solutions to the analytical differential equations provided by Greenspan $[3,4,7,15]$ as given by,

$$
V(r)=R+2 \sum_{n=1}^{\infty}\left[J_{1}\left(j_{n} R\right) / j_{n} J_{0}\left(j_{n}\right)\right] \exp \left(-j_{n}^{2} \nu t_{0} / a^{2}\right)
$$




$$
\frac{\partial V}{\partial R}=1+\sum_{n=1}^{\infty}\left[1-J_{2}\left(j_{n}\right) / J_{0}\left(j_{n}\right)\right] \exp \left(-j_{n}^{2} \nu t / a^{2}\right),
$$

where $R$ is $r / a, J_{1}$ is the Bessel function of the first kind order $i$ and $j_{n}$ is the $n$th zero of $J_{1}$, and $t_{0}$ is the velocity time change. Equation 2.8 and 2.9 finds the velocity profile and wall shear stress for an impulsively started cylinder when $t=0$. Equations for varied velocity change can be seen in the MATLAB script in the appendices.

\subsection{CFD-Finite Volume Method}

Fluent is a finite volume method that simultaneously calculates governing equations at each cell or element. An important reason to use Fluent as the CFD software choice is the ability to use the Volume of Fluid (VOF) model, which can model two or more immiscible fluids using a single set of momentum equations to track the volume fraction of each fluid during flow. This allows Fluent to model and track a two phase flow of gas and liquid in the containers. The VOF has a few limitations such as using the pressure-based solver in Fluent instead of a density-based solver and a second order implicit solver cannot be used with the explicit solver. An explicit differencing scheme was used for the volume of fraction parameters [29]. The equation to track the volume of fractions is as follows:

$$
\frac{1}{\rho_{q}}\left[\frac{\partial}{\partial t}\left(\alpha_{q} \rho_{q}\right)+\nabla \cdot\left(\alpha_{q} \rho_{q} \vec{v}_{p}\right)=S_{\alpha q}+\sum_{p=1}^{n}\left(\dot{m}_{p q}-\dot{m}_{p q}\right)\right]
$$

where $\dot{m}_{q p}$ is the mass transfer from phase $q$ to $p, \dot{m}_{p q}$ is the mass transfer from phase $p$ to $q, \rho_{q}$ is the density at the $q$ th phase, $\alpha$ is the volume fraction cell for the $p$ th and qth phase. 
The following equation constrains the volume of fraction equation as

$$
\begin{gathered}
\sum_{q=1}^{n} \alpha_{q}=1, \\
\alpha_{1}+\alpha_{2}=1,
\end{gathered}
$$

where $\alpha_{q}$ is noted as the volume fraction cell and $q$ is the fluid phase number. Equation 2.11 restrict the sum of the phases to be equal to one. In our model, Equation 2.12 shows the two phases of $\theta_{1}$ and $\theta_{2}$ in our model as gas and liquid, respectively.

Table 2.1 - Solution method setup used in Fluent.

\begin{tabular}{|c|c|}
\hline Name & Type \\
\hline \hline Scheme & SIMPLE \\
\hline Gradient & Least Squares Cell Based \\
\hline Pressure & PRESTO! \\
\hline Momentum & Second Order Upwind \\
\hline Swirl Velocity & Second Order Upwind \\
\hline Volume Fraction & Compressive \\
\hline
\end{tabular}

The solution methods setup for Fluent is shown in Table 2.1. The SIMPLE scheme is used for the pressure-velocity coupling for a faster convergence. Since the model is an unstructured mesh, transient, and not using large body forces, the least squares cell based gradient and the PRESTO! (PREssure STaggering Option) parameters are chosen. The least squares cell based gradient is used to discretize the convection and diffusion terms in the momentum equations [29]. A second order upwind is chosen for both momentum and swirl velocity equations for a higher order of accuracy.

For the spin-stop cases, the 2-D mesh was initially developed using a serial processor from a personal computer, which causes long computational time for finer meshes. 
The personal computer was done on an Intel Core i7-6500 processor with 16 GB of RAM and a Nvidia Geforce MX150 (GPU). In order to decrease the computational time and increase efficiency, parallel processing was used to simultaneously compute the model using different core processors instead of a single processor using serial. Parallel processing was made possible by using a high performance computing system called HPC Bishop Cluster from Cal Poly's Aerospace Engineering department. The HPC Bishop Cluster has 4 servers with each server running on two 8 x Intel Xeon 12 core processors with 256 GB of dynamic RAM and 8 x Nvidia Tesla K80 (GPU).

The approximate computational time differences between a personal computer and the HPC Bishop Cluster is shown in Figure 2.2. There is a noticeable difference in computational time as 2-D axisymmetric coarse meshes can be computed within 15 minutes when using the HPC Bishop Cluster. The 3-D cases can be computed within two days in comparison to the 14 days on a personal computer. Without the use of a high performance computing system, the computational time for a fine mesh of a 2-D case will increase from three days to thirty days or more. 
Table 2.2 - Computational time difference between personal computer and Bishop cluster.

\begin{tabular}{|c|c|c|}
\hline Mesh Туре & $\begin{array}{c}\text { Personal } \\
\text { Computer } \\
\text { Time }\end{array}$ & $\begin{array}{c}\text { HPC } \\
\text { Bishop } \\
\text { Cluster } \\
\text { Time }\end{array}$ \\
\hline $\begin{array}{c}2-\mathrm{D} \\
\text { Axisymmetric } \\
\text { Coarse }\end{array}$ & 2 hours & $\begin{array}{c}15 \\
\text { minutes }\end{array}$ \\
\hline $\begin{array}{c}2-\mathrm{D} \\
\text { Axisymmetric } \\
\text { Fine }\end{array}$ & 30 days & 5 days \\
\hline 3-D Coarse & 14 days & 2 days \\
\hline
\end{tabular}

\subsubsection{Validation Model}

A validation model was first created to compare against analytical models as well as show mesh convergence.

\subsubsection{Setup}

The first step involves creating the 2-D axisymmetric cylinder mesh as shown in Figure 2.7, which is a mirrored mesh for visual purposes. The nodes are finer along the boundary walls of the container to capture the boundary layer behavior by using a growth factor. A growth factor ratio of 1.005 was used for the initial model with 6360 nodes. 

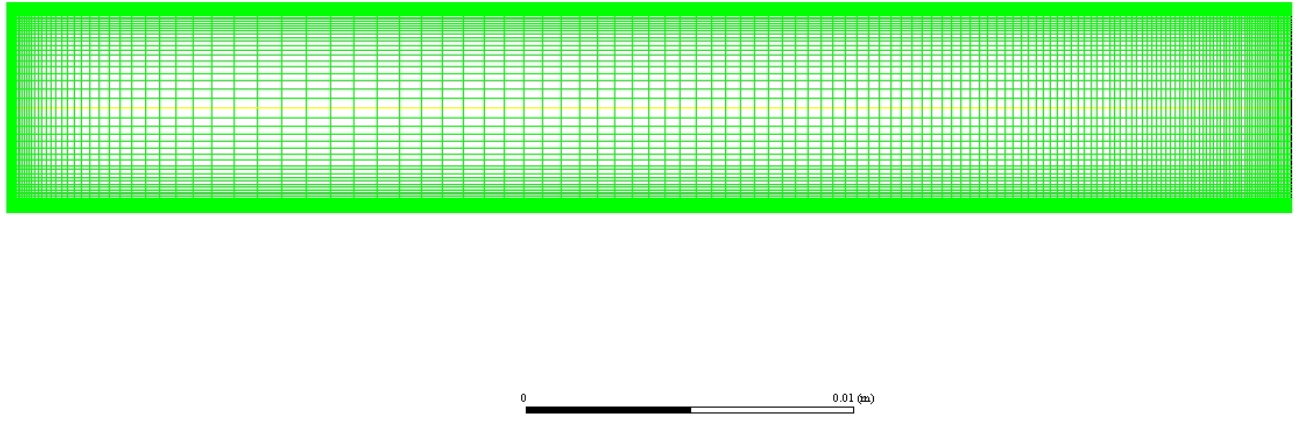

Figure 2.7 - An image of a mirrored 2-D axisymmetric mesh.

The mesh is exported into ANSYS Fluent using the VOF model for a 2-D axisymmetric swirl. In Fluent, the mesh is symmetric about the x-axis; hence, the gravity is in the positive x-direction as shown in Figure 2.8. Also, the containers spin vertically, but Fluent spins about $x$-axis for 2 -D axisymmetric models.

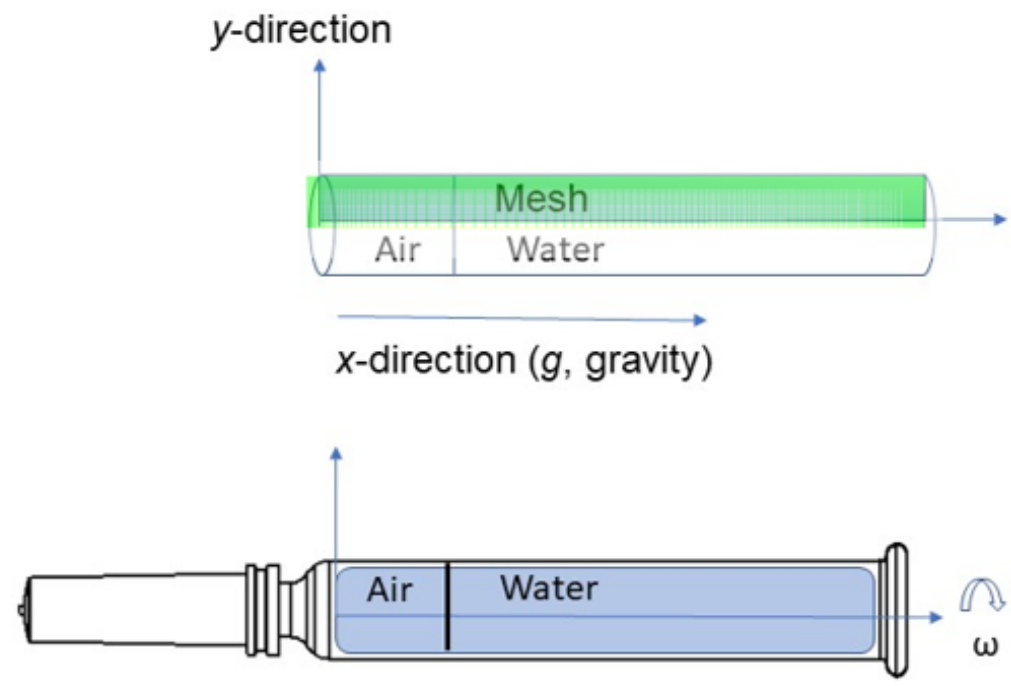

Figure 2.8 - A diagram comparison between the created 2-D mesh using Fluent's axisymmetric coordinate with the physical geometry. 
For the solution to reach convergence, a Courant-Friedrichs-Lewey (CFL) condition is calculated to find the time step $\delta t$. The CFL condition gives an approximate timestep value for the models to not have a divergent solution. The equation for 2-D CFL condition is

$$
\frac{U \triangle t}{\triangle x} \leq C_{\max },
$$

where $U$ is the velocity, $\triangle t$ is the time step, $\triangle X$ is the mesh size, and $C_{\max }$ is the dimensionless maximum Courant number. In Equation 2.13, the Courant number, $C_{\max }$, is assumed to be 1 for numerical stability using the explicit computational method. Any value more than 1 would cause the computations at each cell and time step to diverge and become unstable.

Table 2.3 - Validation Model Parameters

\begin{tabular}{|c|c|c|}
\hline Angular Velocity & 188.6 & $\mathrm{rad} / \mathrm{s}$ \\
\hline Contact Angle & 135 & degrees \\
\hline Surface Tension & 0.072 & $\mathrm{~N} / \mathrm{m}$ \\
\hline Fill Volume & 1 & $\mathrm{~mL}$ \\
\hline
\end{tabular}

The validation model's parameters are shown in Table 2.3, which spins up to a speed of $188.6 \mathrm{rad} / \mathrm{s}$ or $1800 \mathrm{rpm}$ with assumed surface tension and contact angle of $0.072 \mathrm{~N} / \mathrm{m}$ and 45 or 135 degrees based on the Fluent theory guide [29]. These parameters were also chosen because the MATLAB analytical model for meniscus height was able to correctly iterate to find a solution. The fill volume for the water is $1 \mathrm{~mL}$ in a $1 \mathrm{~mL}$ syringe plastic container. The spin-up and spin-down velocity change is 2.5 milliseconds. The container spins up for 5 seconds and spins down for 5 seconds. The Reynolds number for the validation model was $R e=1900$. Since the the Reynolds number was much less than the critical Reynolds number of $3 \mathrm{E} 10^{5}$, 
the laminar model was used for the validation model. All subsequent cases used the laminar model since the Reynolds number range was 100-5500.

Using Equation 2.14 with the validation model parameters, the time step calculated to be 0.0015 seconds. Since this is the maximum time step size to complete the simulation without diverging, the time step increases to 0.0005 seconds to gain a better insight into the time intervals during a spin-up time of 0.0025 seconds. Increasing this time steps size reduces the error when computing the mesh convergence study for four different meshes, in which the finer the mesh, the finer the time step.

\subsubsection{Analytical Solutions for Validation Model}

This section details the MATLAB analytical solutions of the meniscus height, velocity profile, and wall shear stress for the validation model.

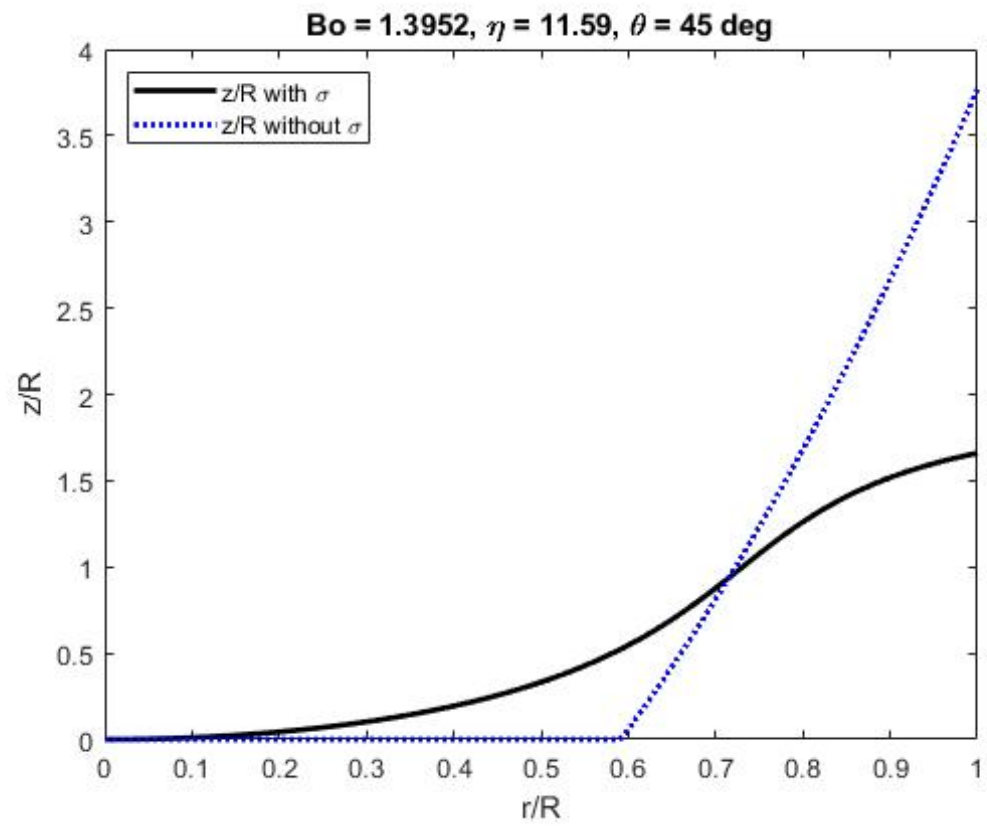

Figure 2.9 - The shape of the surface profile with corresponding Bond number, eta, and contact angle. 
Figure 2.9 shows the surface profile for the validation model with a Bond number, $B o=1.3952, \eta=11.59$, and contact angle, $\theta=45$ degrees. The black line, $z / R$, shows the surface profile with the presence of surface tension whereas the dotted blue line shows the surface profile without surface tension. The meniscus height is $h=5.58 \mathrm{~mm}$ since the $h_{0} / R=0.8682$ and $h$ is twice the measurement of $h_{0}$ because it needs to take into account for the diameter of the container.

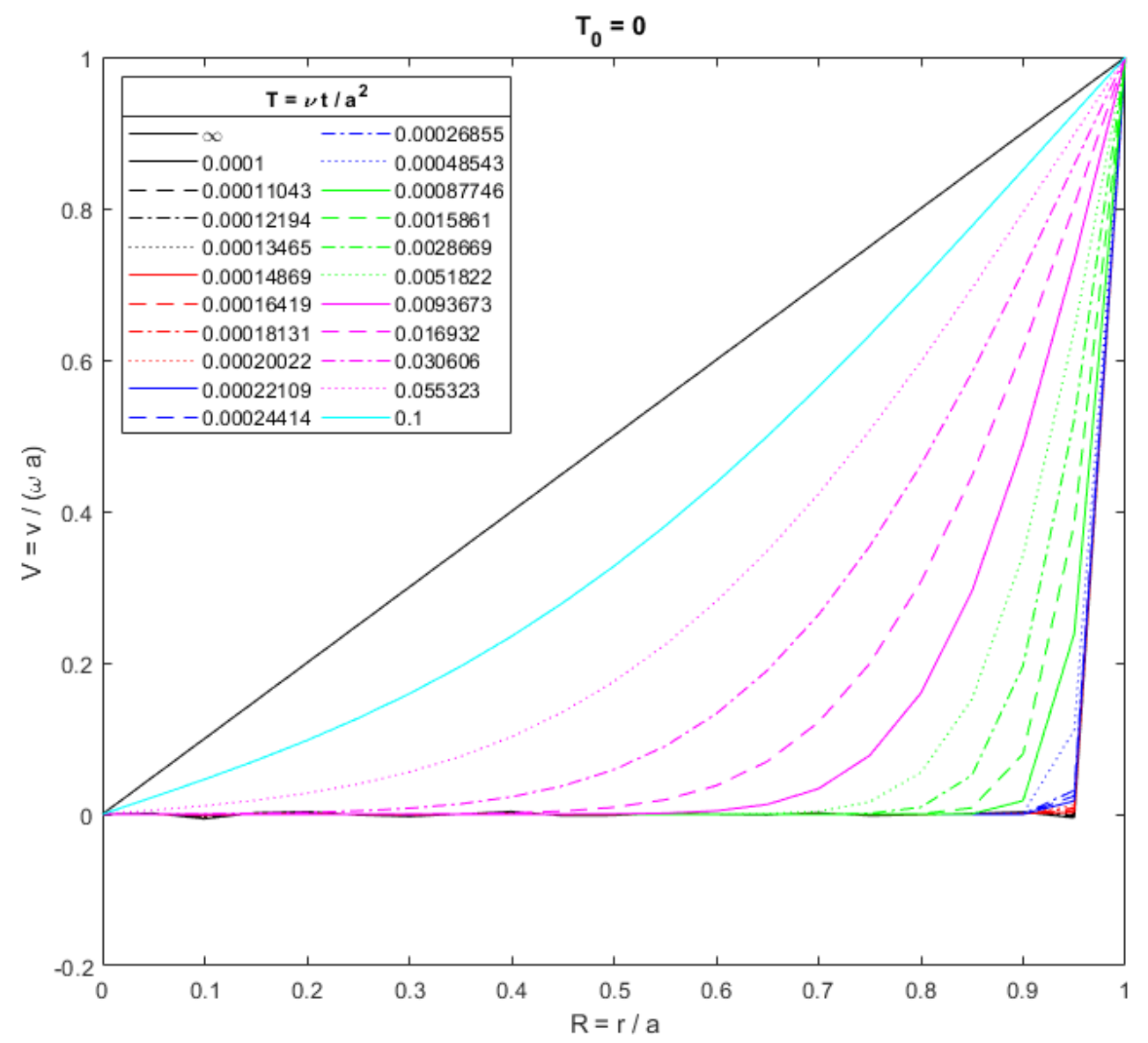

Figure 2.10 - Analytical solutions of velocity profiles for an infinite cylinder with no velocity change. 
For the acceleration study, the validation model for an impulsively started infinite cylinder when $T_{0}$ is shown in Figure 2.10. The velocity profiles show how they change from infinity to 0.1 seconds. At infinity, the velocity profile is linear, whereas in reality, the velocity profile will approach the linear profile as shown for other dimensionless times.

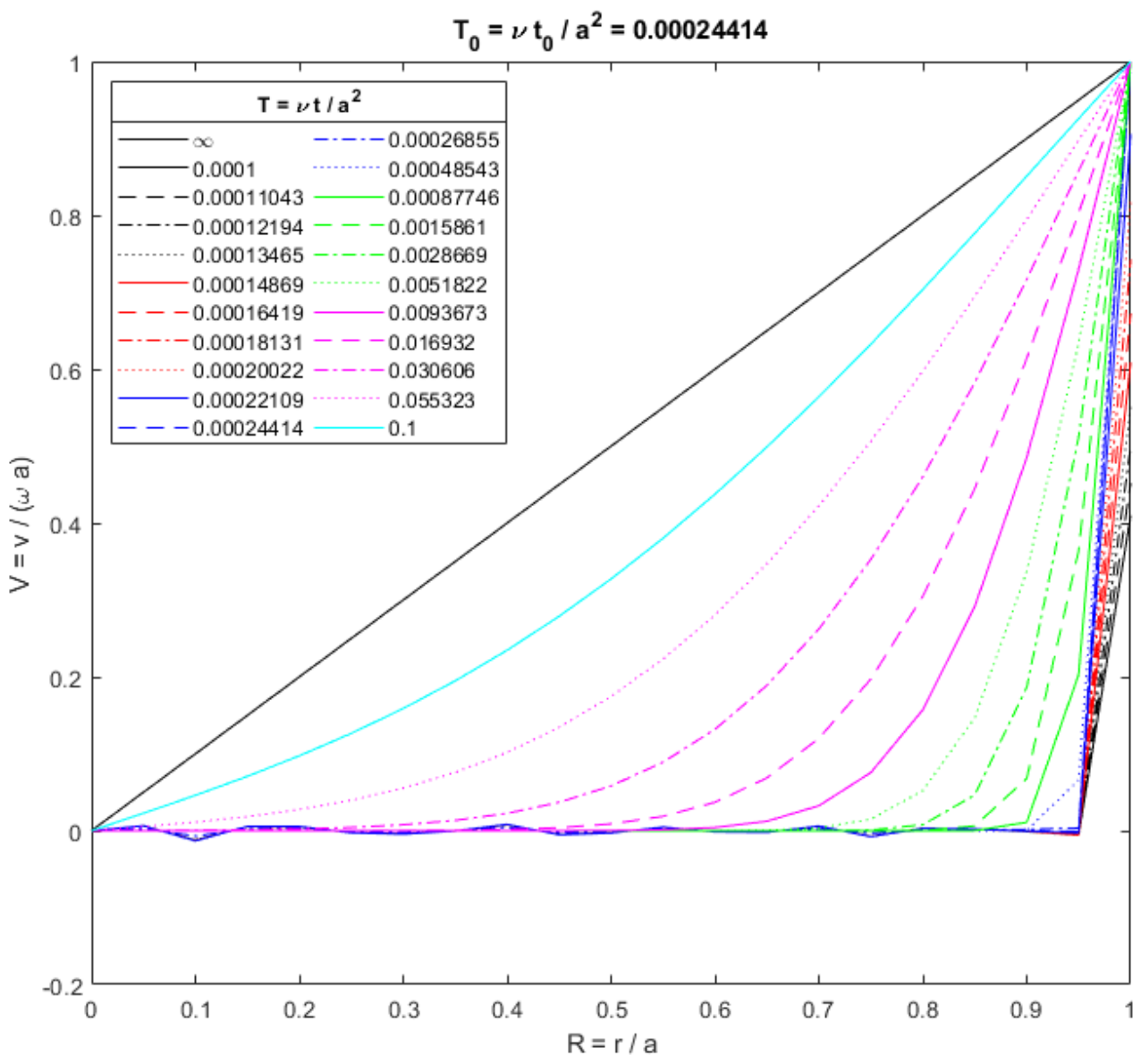

Figure 2.11 - Analytical solutions of velocity profiles for an infinite cylinder with velocity change at outer wall.

When a velocity change occurs, Figure 2.11 shows the velocity profile for a velocity change of 2.5 milliseconds. The plot shows a more gradual slope change in comparison to Figure 2.10. In comparison with the analytical model, the validation Fluent model for a $1 \mathrm{~mL}$ syringe at $1800 \mathrm{rpm}$ for velocity profile is shown in Figure 2.12. The 
velocity profiles do not show the same linear profile as in Figure 2.11, but show the same trend to achieve a linear velocity profile. This makes sense since the analytical model is used for an infinite cylinder.

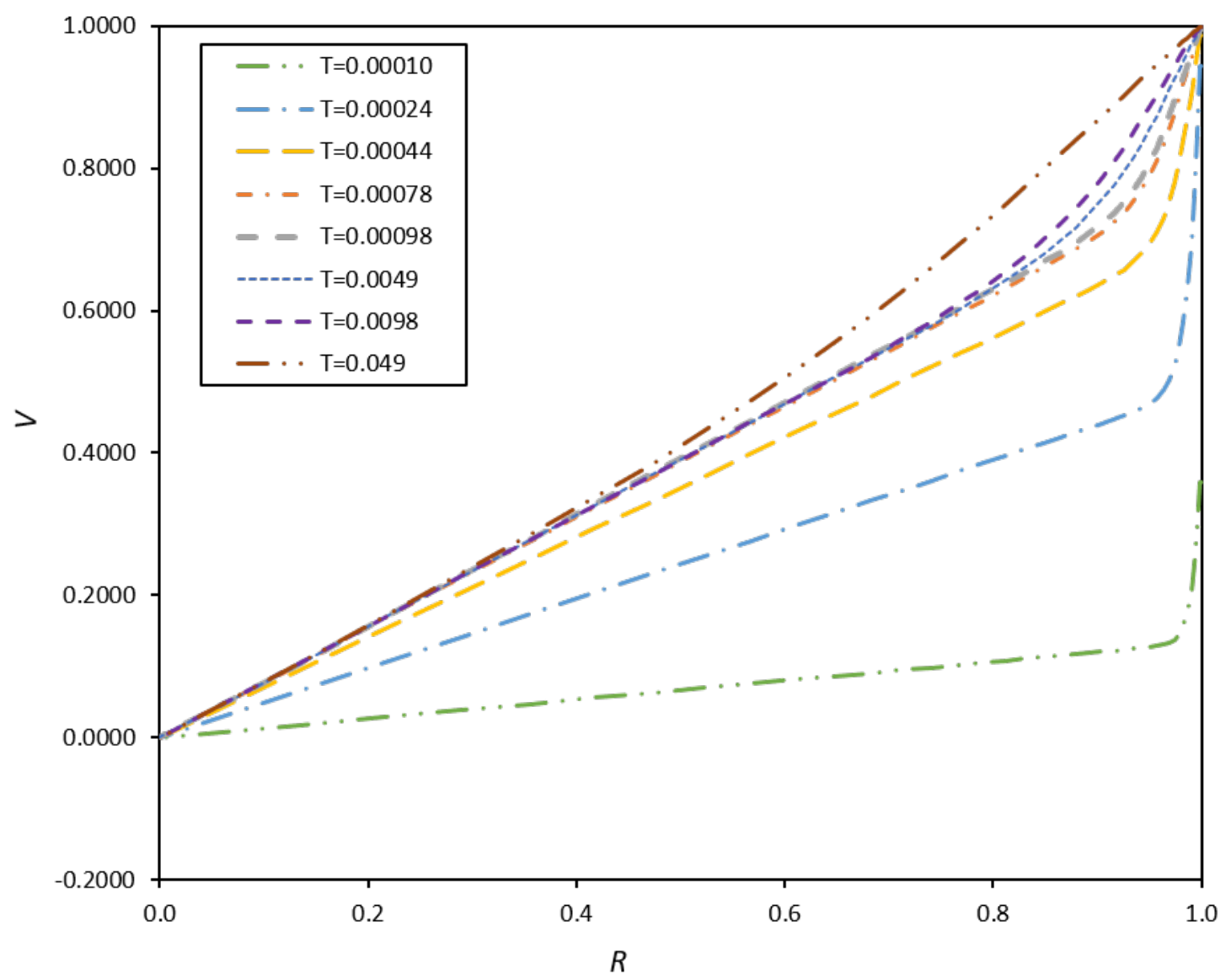

Figure 2.12 - Velocity profiles for the Fluent model at $1800 \mathrm{rpm}$. 


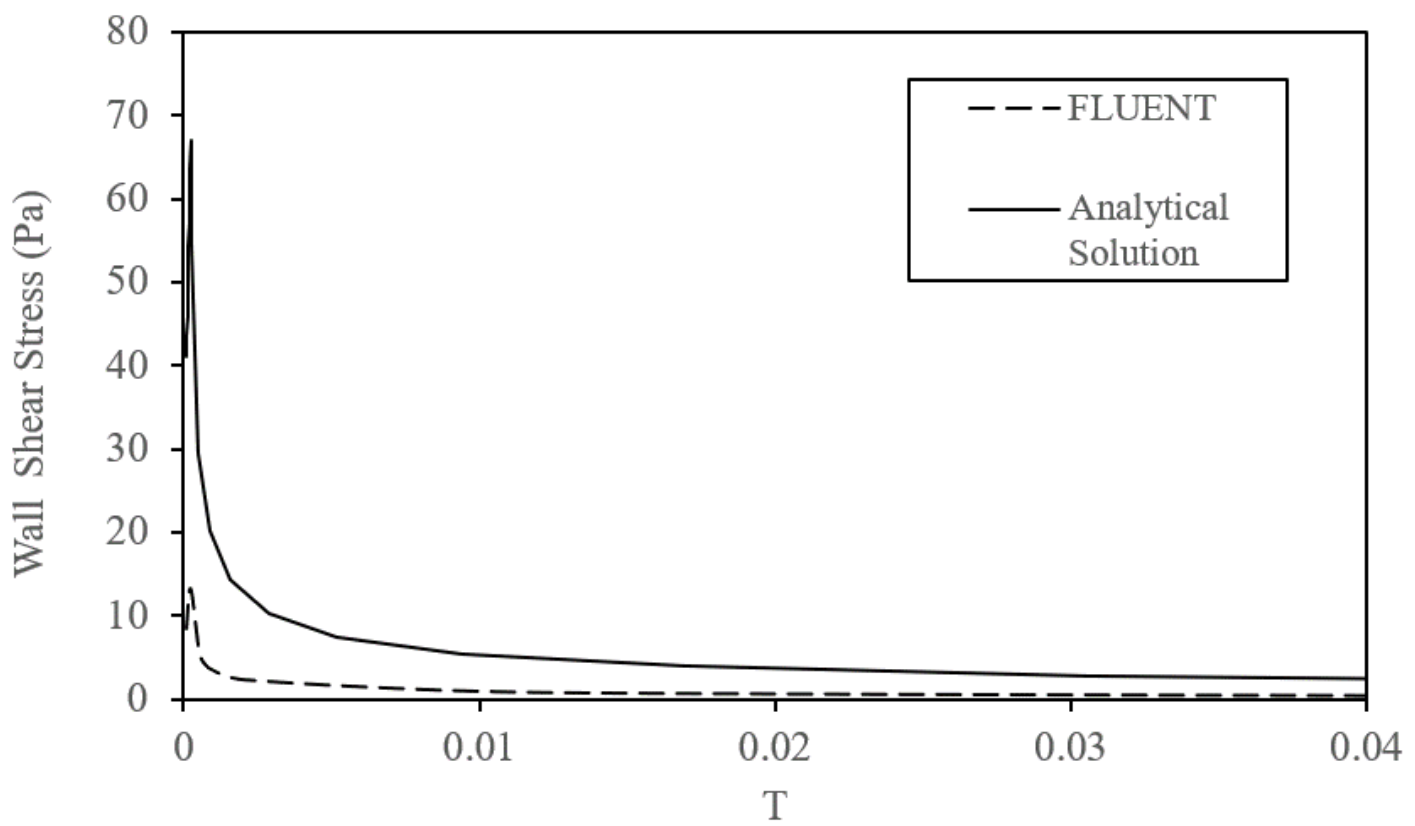

Figure 2.13 - Average Wall shear stress for the Fluent model with analytical solution

As shown in Figure 2.13, the Fluent solution maximizes at the same point in time as the analytical solution at $T=0.0001$. The Fluent solution also decays in a similar fashion as the analytical solution. There is a noticeable difference between wall shear stress for the analytical and Fluent solution at $68 \mathrm{~Pa}$ and $15 \mathrm{~Pa}$, respectively. The wall shear stress difference can be attributed to the analytical solution used for an infinite cylinder. 


\subsubsection{Mesh Convergence Studies}

Table 2.4 - Mesh sizes with growth factors for the mesh convergence studies.

\begin{tabular}{|c|c|c|}
\hline Name & Nodes & Growth Factor \\
\hline \hline Mesh A & 3175 & 1.005 \\
\hline Mesh B & 6360 & 1.005 \\
\hline Mesh C & 12750 & 1.004 \\
\hline Mesh D & 25600 & 1.003 \\
\hline
\end{tabular}

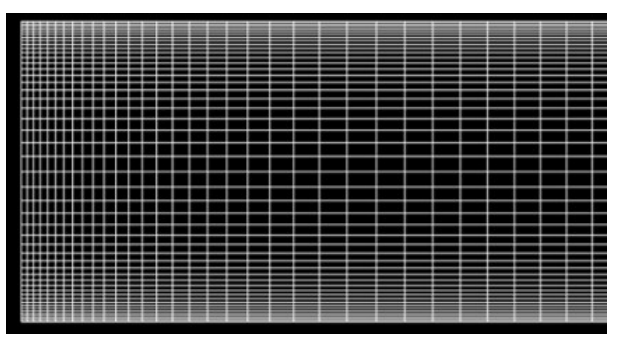

(a) Mesh A

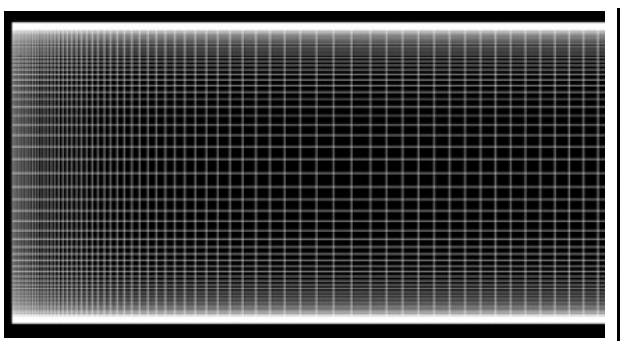

(c) Mesh C

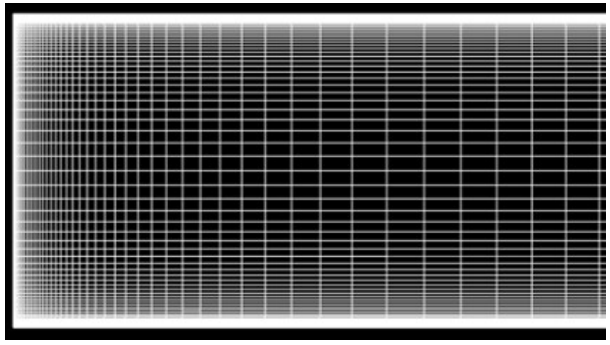

(b) Mesh B

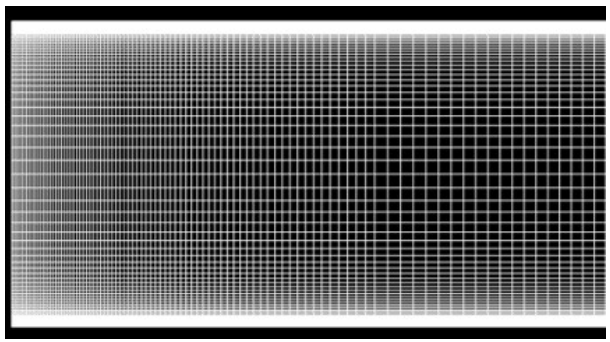

(d) Mesh D

Figure 2.14 - Images of the four 2-D meshes for the mesh convergence studies.

Four meshes were created for the mesh convergence study. The number of nodes and growth factor are shown in Table 2.4. Mesh A is the coarse mesh at 3175 nodes with a growth factor of 1.005 , Mesh B is the fine mesh at 6360 nodes with a growth factor of 1.005, Mesh $\mathrm{C}$ is the finer mesh at 12750 nodes with a growth factor of 1.004, and Mesh D is the finest mesh at 25600 nodes with a growth factor of 1.003. In all 
cases, a geometrically growing fine mesh is created along the walls to account for the boundary layer with growth factors between 1.003 to 1.005 . Figure 2.14 shows the mirrored mesh about the $x$-axis with a close up of the upper half of the container.

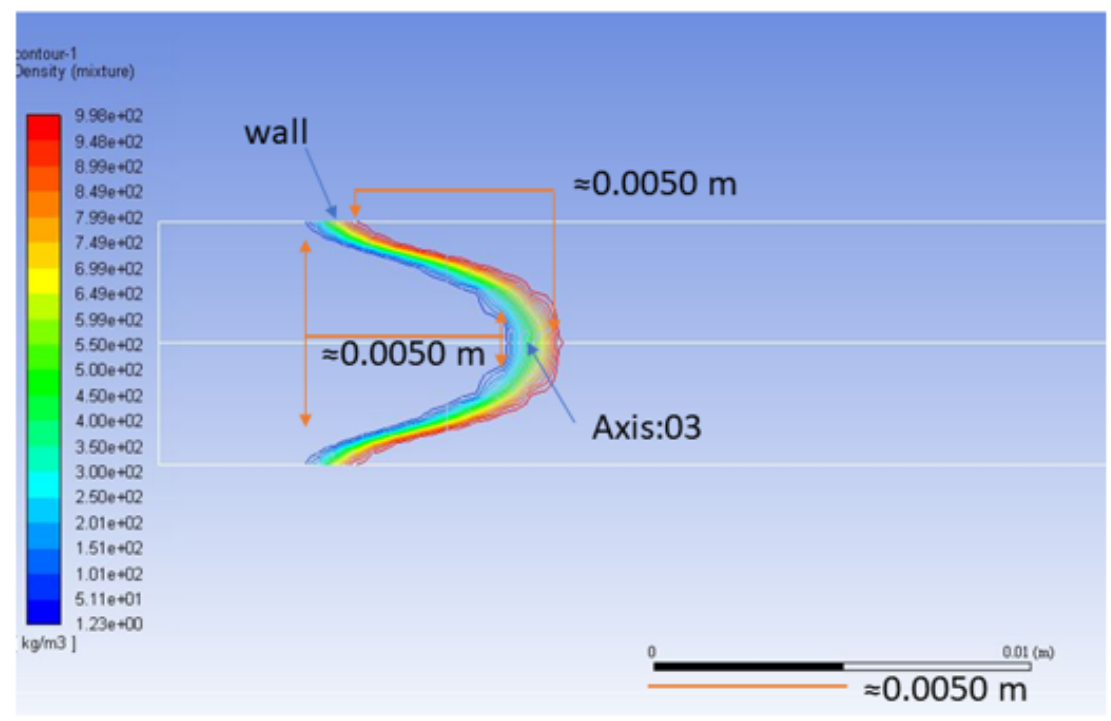

Figure 2.15 - Density contour plot with approximate meniscus height in Fluent.

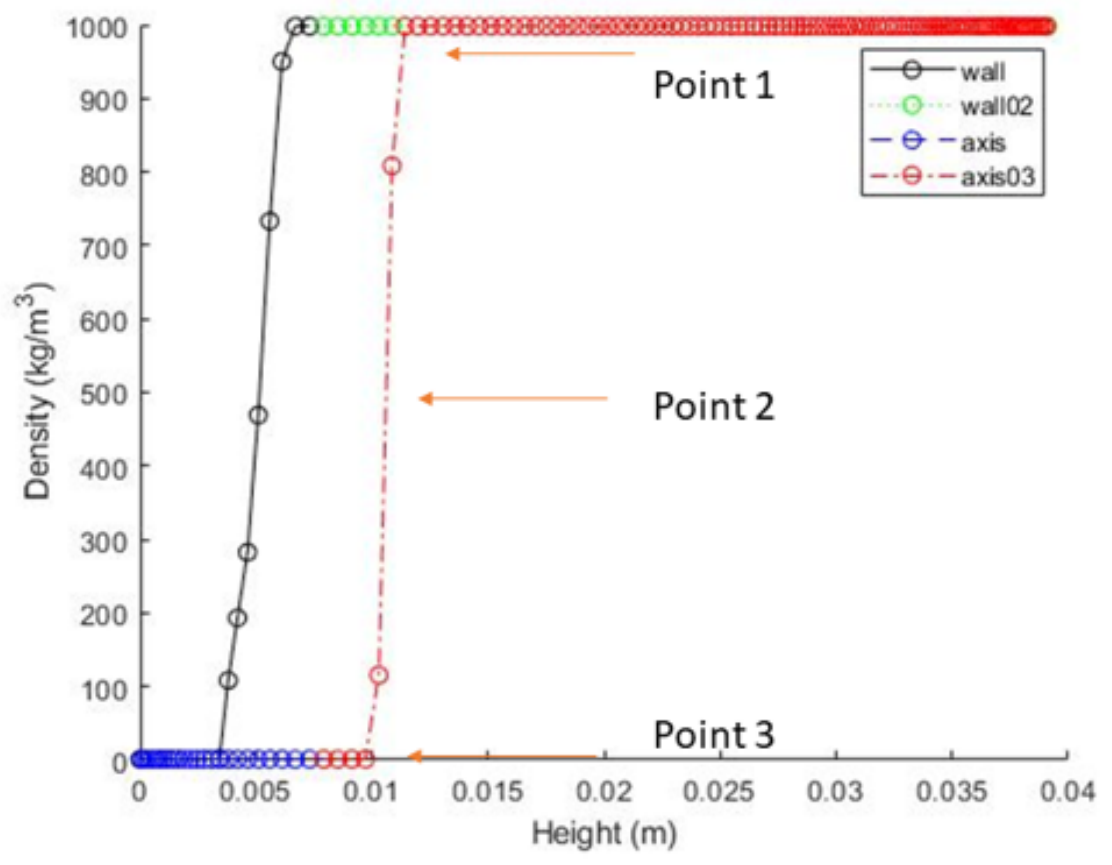

Figure 2.16 - A diagram of a MATLAB plot to determine the meniscus height. 
The validation model's density contour plot with the meniscus height is shown in Figure 2.15. The meniscus height was initially found by inspection from Figure 2.15 as $0.0050 \mathrm{~m}$. Since the meniscus height cannot be accurately determined by inspection, the meniscus height for each mesh was approximated by exporting the density results from Fluent into MATLAB. Using the density data, an average meniscus height was calculated using the diagram with the MATLAB plot in Figure 2.16. The "wall" represents the wall touching the initial gas zone and "axis:03" represents the axis touching the initial liquid zone as shown in Figure 2.15. At three density points of 0, 500, and $1000 \mathrm{~kg} / \mathrm{m}^{3}$, the meniscus height was calculated and averaged to find the maximum meniscus height for the validation model. The meniscus height for all meshes were calculated in the same manner.

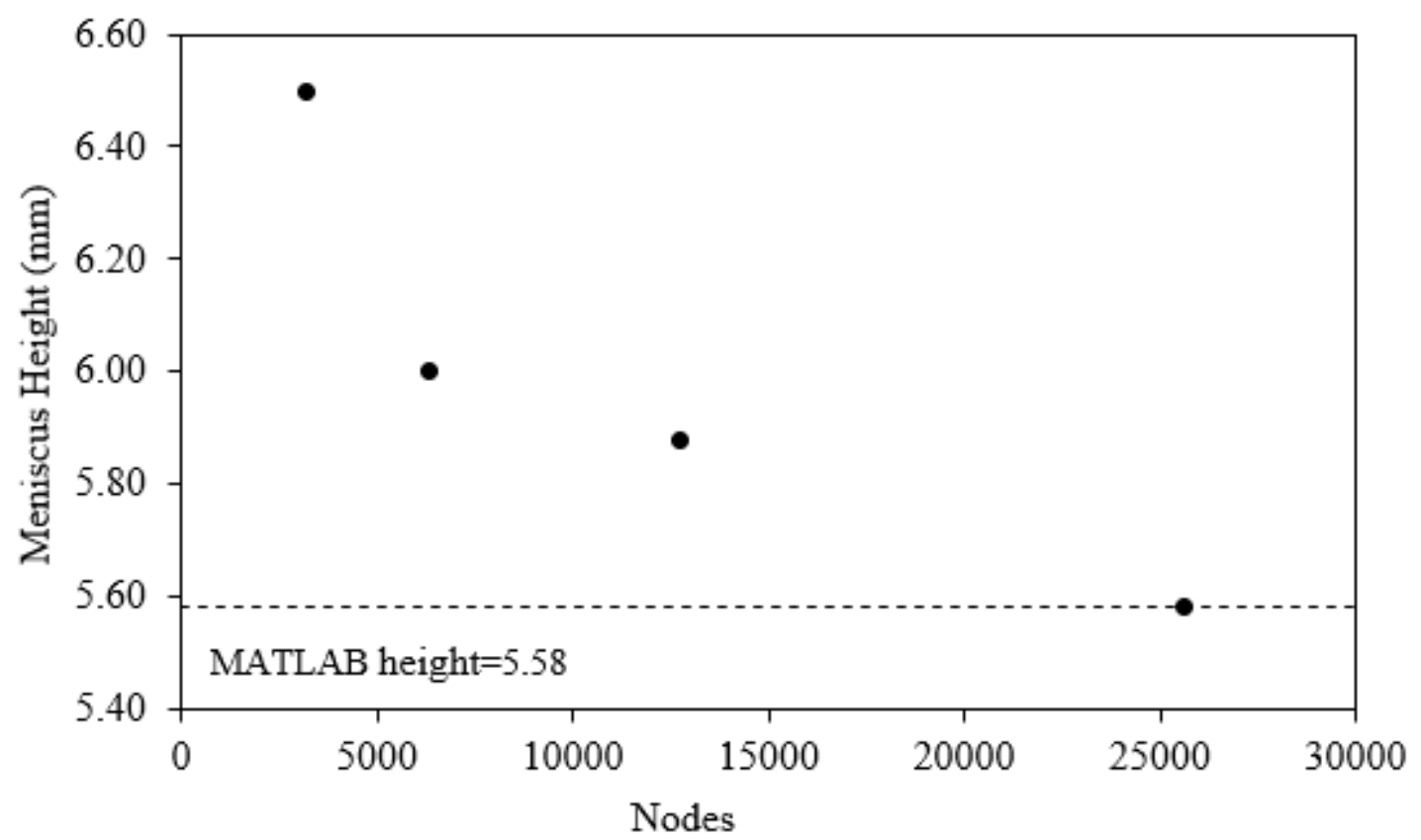

Figure 2.17 - Mesh convergence plot for density height across mesh nodes at $t=5$ seconds.

A result of the meniscus heights for the mesh A through D are shown in Figure 2.17. Figure 2.17 shows an agreement that the finer the mesh, the closer the density 
height reaches the MATLAB solid body rotation height. It is important to note the time step size for Mesh D at 25600 nodes was at 0.00005 seconds. The time step size increased from the 0.0005 to 0.00005 seconds because Mesh D diverged at a time step size of 0.0005 seconds. The other meshes were not calculated at 0.00005 seconds due to computational time. This introduces an iteration error since mesh D underwent with more iterations in comparison to meshes $\mathrm{A}, \mathrm{B}$, and $\mathrm{C}$.

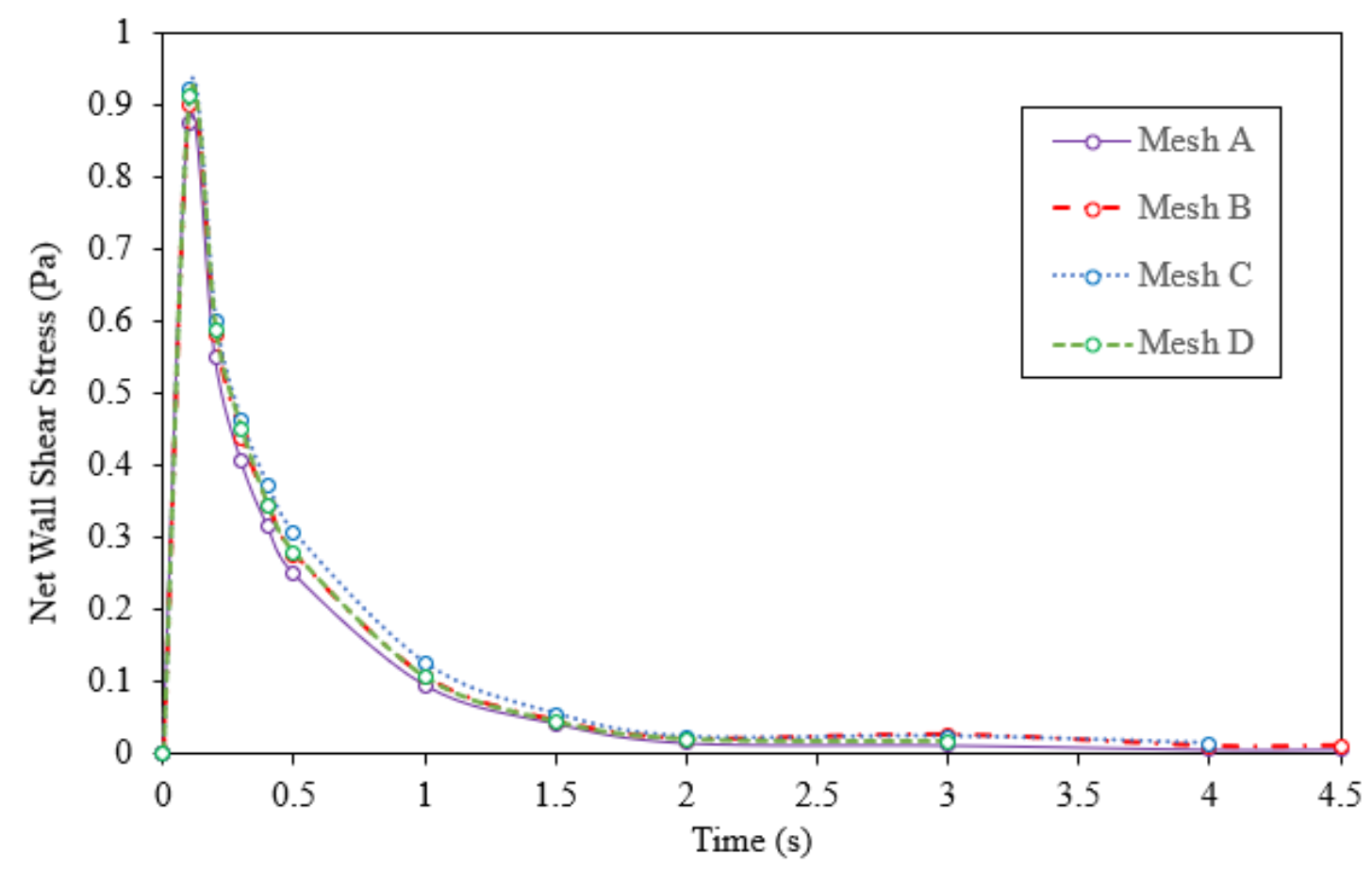

Figure 2.18 - Mesh convergence plot for net wall shear stress over time.

Figure 2.18 shows the mesh convergence plot for net wall shear stress over time in pascals for the four different meshes. The plot shows the highest wall shear stress occurs at a maximum of approximately $0.95 \mathrm{~Pa}$ at 0.1 seconds and decays until it reaches steady state in between 2.5 and 3 seconds. The predicted spin-up time criterion was 2.85 seconds based on Equation 1.13, which shows a good agreement with Figure 2.18. 


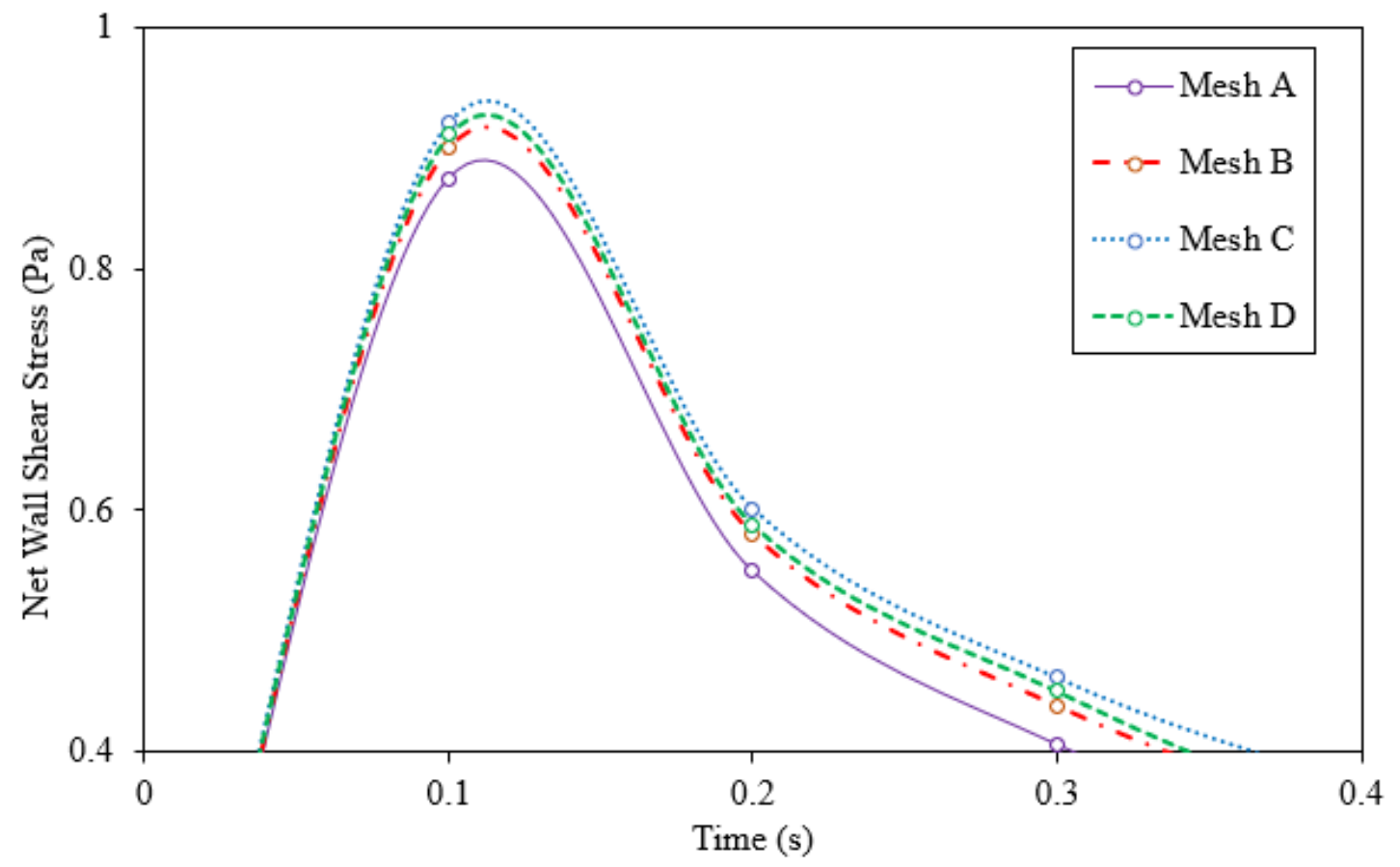

Figure 2.19 - A plot of a mesh convergence closeup of Figure 2.18b.

Figure 2.19 shows a close up of the mesh convergence in Figure 2.18 to further illustrate the differences in the wall shear stress. Upon closer inspection, Mesh C is slightly higher than Mesh D, which can be attributed to the difference in growth factor at the upper wall. Based on the results, reasonable mesh convergence was obtained for mesh B, which was used for all subsequent calculations.

\subsubsection{2-D Axisymmetric Model Studies}

Since the initial model was validated, several studies of different containers and process parameters were conducted. The meshes were further refined to resemble the physical fluid domain by rounding the corners. The new meshes for a $5 \mathrm{~mL}$ cartridge and $2.25 \mathrm{~mL}$ syringe are shown in Figure 2.20 and 2.21. 

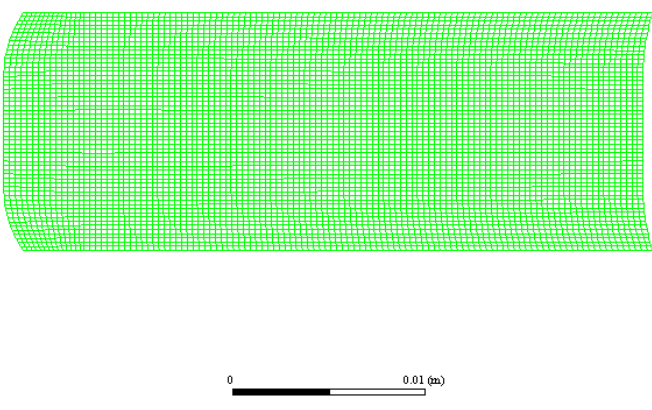

Figure 2.20 - A mesh for the $5 \mathrm{~mL}$ cartridge.

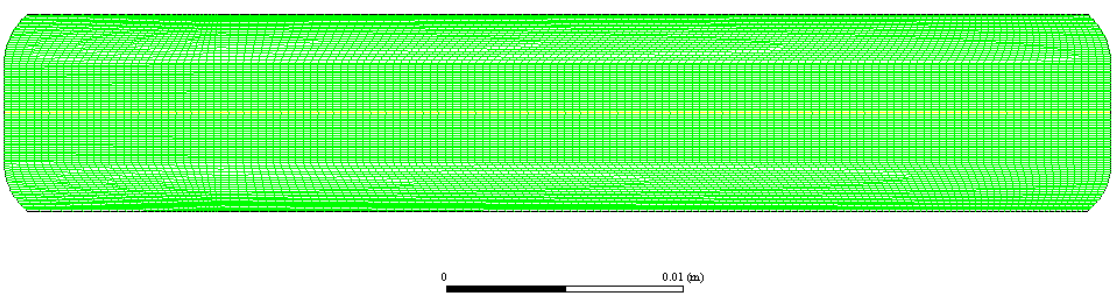

Figure 2.21 - A mesh for the $2.25 \mathrm{~mL}$ syringe 
Table 2.5 - Different viscosity values for $5 \mathrm{~mL}$ cartridge with an angular velocity of $3000 \mathrm{rpm}$.

\begin{tabular}{|c|c|c|c|}
\hline $\begin{array}{c}\text { Viscosity } \\
(\mathbf{c P})\end{array}$ & $\begin{array}{c}\text { Angular } \\
\text { Velocity } \\
\text { (rpm) }\end{array}$ & $\begin{array}{c}\text { Reynolds } \\
\text { Number }\end{array}$ & $\begin{array}{c}\text { Spin-up } \\
\text { Time (s) }\end{array}$ \\
\hline \hline 5 & 3000 & 2376 & 0.79 \\
\hline 10 & 3000 & 1188 & 0.56 \\
\hline 20 & 3000 & 594 & 0.39 \\
\hline
\end{tabular}

Table 2.5 shows varying viscosity values at 5,10 , and 20 centipoise for a $5 \mathrm{~mL}$ cartridge spinning at $3000 \mathrm{rpm}$ with an assumed surface tension of $0.072 \mathrm{~N} / \mathrm{m}$ in Fluent. The change in viscosity values will increase the viscous forces, which would decrease fluid movement and meniscus height.

Table 2.6 shows the CFD simulations for different surface tension values at 0.048 , 0.072 , and $0.096 \mathrm{~N} / \mathrm{m}$ for a $2.25 \mathrm{~mL}$ syringe spinning with a $2 \mathrm{~mL}$ fill at $4000 \mathrm{rpm}$. The dynamic viscosity for all simulations is $0.039 \mathrm{~kg} / \mathrm{m}-\mathrm{s}$ or $39 \mathrm{cP}$ and a density of $1130 \mathrm{~kg} / \mathrm{m}^{3}$. These simulations will show whether surface tension has a tremendous effect on the spin-stop process.

Table 2.6 - Different surface tension values for a $4000 \mathrm{rpm} 2.25 \mathrm{~mL}$ syringe.

\begin{tabular}{|c|c|}
\hline Number & Surface Tension(N/m) \\
\hline \hline 1 & 0.048 \\
\hline 2 & 0.072 \\
\hline 3 & 0.096 \\
\hline
\end{tabular}




\subsubsection{Setup}

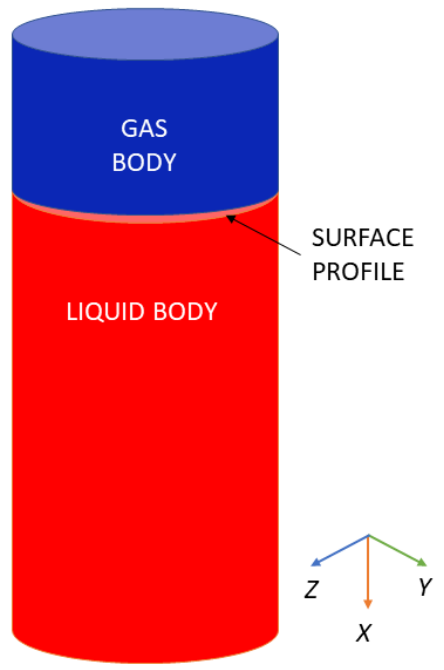

Figure 2.22 - A Diagram of the solid bodies in a 3D mesh.

The 3-D model is set up similar to the 2-D case except for the axis boundary condition. The mesh was created in ICEM, in which bodies are created to represent the gas and liquid zones. Two meshes were created to represent the $1 \mathrm{~mL}$ syringe and $5 \mathrm{~mL}$ cartridge. A diagram shows how the bodies in the mesh were represented as gas and liquid zones in Figure 2.22. The $1 \mathrm{~mL}$ syringe is shown in Figure 2.23a. The $5 \mathrm{~mL}$ cartridge mesh is shown in Figure 2.23b. A closer view of the meshes are shown in Figure 2.24.

For the 3-D models, several cases were simulated. The initial case involved simulating the validation model of a water filled syringe at $1800 \mathrm{rpm}$. Similar to the 2-D cases, the mesh is finer near the walls to account for the boundary layer. All boundary and initial conditions are the same from the 2-D models for the $3-\mathrm{D}$ models. The 
time step for the 3-D model was chosen based on the CFL condition for 3-D cases, which is

$$
\frac{U_{x} \triangle t}{\triangle x}+\frac{U_{y} \triangle t}{\triangle y}+\frac{U_{z} \triangle t}{\triangle z} \leq C_{\max }
$$

where $U_{x}$ is the velocity in the $x$-direction, $U_{y}$ is the velocity in the $y$-direction, and $U_{z}$ is in the $z$-direction. Velocity is only present in the $x$ and $y$ direction; therefore, the CFL condition simplifies to a 2D equation. The time step for the $3 \mathrm{D}$ condition is 0.0005 seconds. 


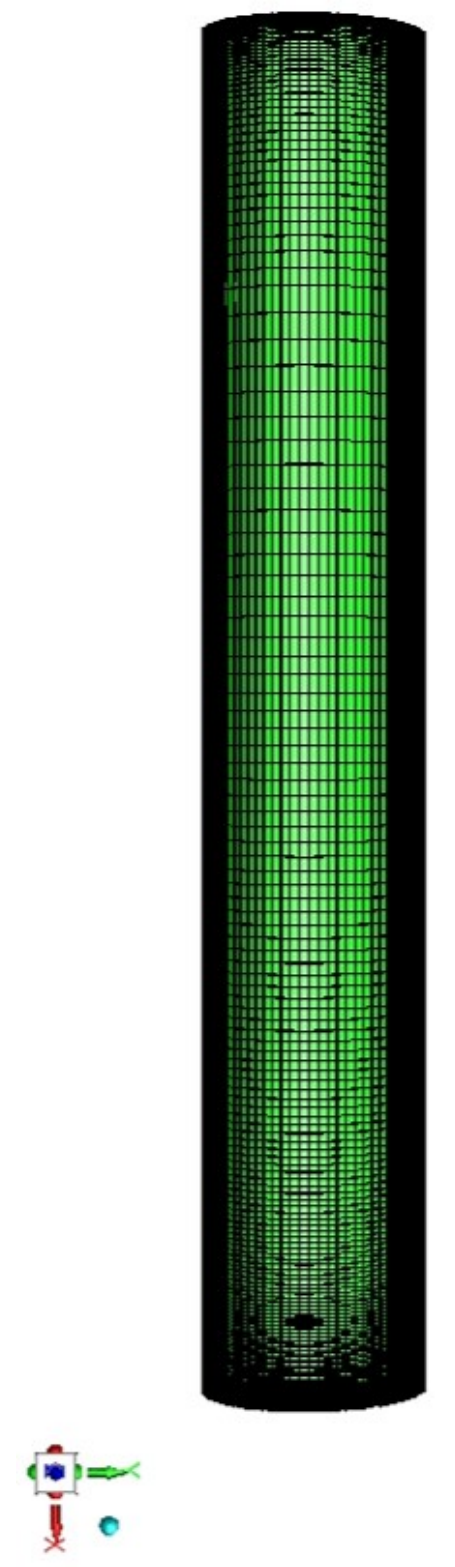

(a) Vertically positioned 3-D mesh for a $1.5 \mathrm{~mL}$ syringe
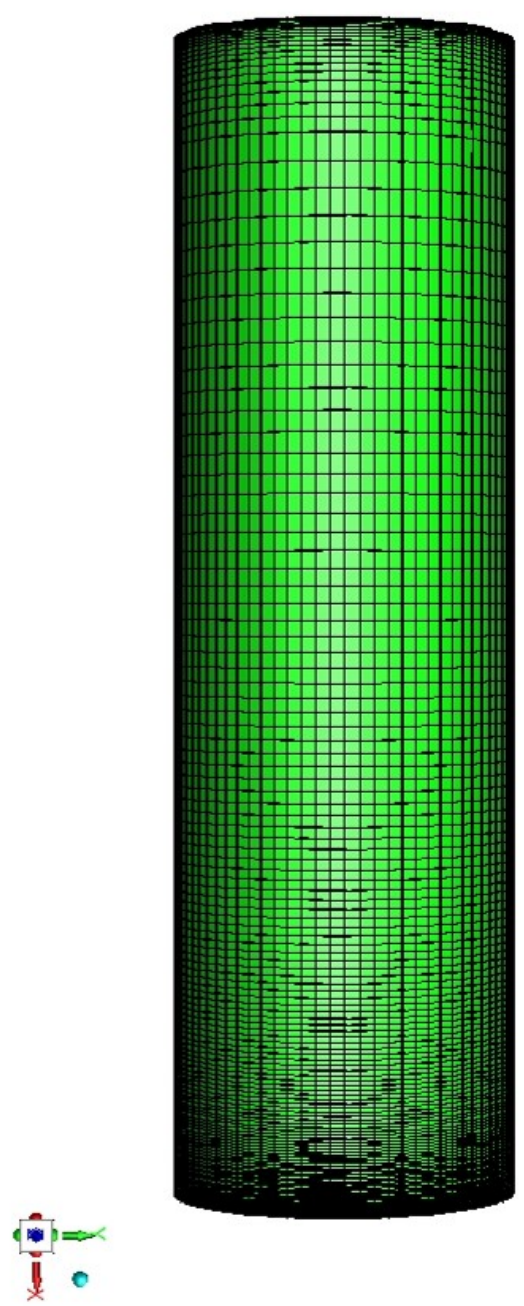

(b) Vertically positioned for a $5 \mathrm{~mL}$ cartridge

Figure 2.23 - 3-D mesh for a $1.5 \mathrm{~mL}$ syringe and $5 \mathrm{~mL}$ cartridge. 


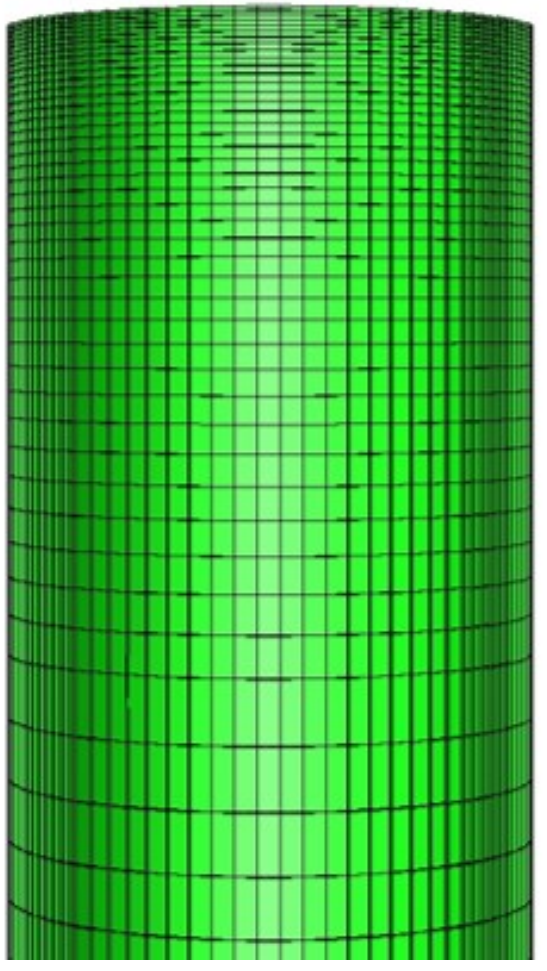

(a) A close up of the vertically positioned 3-D mesh for a $1 \mathrm{~mL}$ syringe

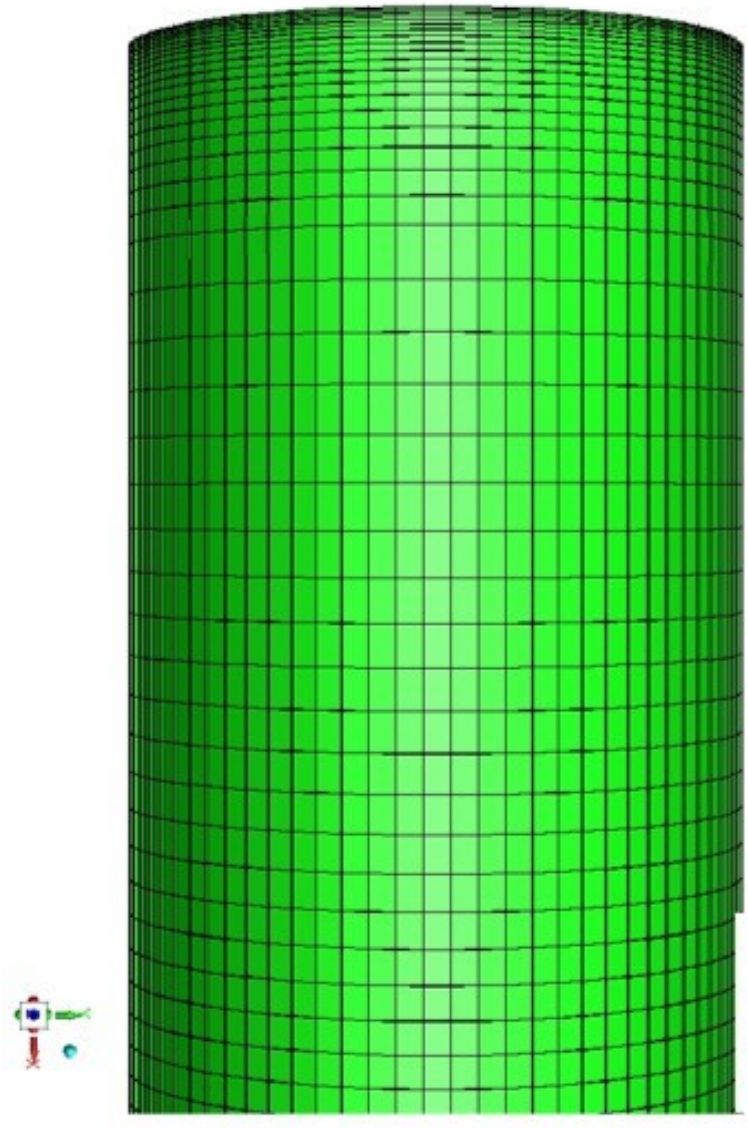

(b) A close up of a vertically positioned 3-D mesh for a $1 \mathrm{~mL}$ cartridge

Figure 2.24 - Close up view for a $1 \mathrm{~mL}$ syringe and a $5 \mathrm{~mL}$ cartridge. 


\subsubsection{Studies}

Table 2.7 describes the involved studies for 3-D simulations. Case 1 is used to validate the analytical MATLAB models and observe any discrepancies between the 2-D axisymmetric case. Case 2 compares the 2-D axsiymmetric case at a higher angular velocity when the meniscus reaches the bottom of the container. Case 3 is used to compare the experimental samples and observe any differences between the meniscus heights. Case 1 spins up to 2.5 seconds and spins down 2.5 seconds for a total of 5 seconds. Cases 1 to 3 all spin up to 1.5 seconds and down to 1.5 seconds since the time to reach steady motion happens sooner.

Table 2.7 - 3-D Simulation Cases for Fluent

\begin{tabular}{|c|c|c|c|c|c|}
\hline $\begin{array}{c}\text { Case } \\
\text { No. }\end{array}$ & $\begin{array}{c}\text { Container } \\
\text { Type }\end{array}$ & $\begin{array}{c}\text { Angular } \\
\text { Velocity } \\
(\mathbf{r a d} / \mathrm{s})\end{array}$ & $\begin{array}{c}\text { Dynamic } \\
\text { Viscosity } \\
(\mathbf{c P})\end{array}$ & $\begin{array}{c}\text { Surface } \\
\text { Tension } \\
(\mathbf{N} / \mathbf{m})\end{array}$ & $\begin{array}{c}\text { Fill } \\
\text { Volume } \\
(\mathbf{m L})\end{array}$ \\
\hline \hline 1 & $\begin{array}{c}1 \mathrm{~mL} \\
\text { Syringe }\end{array}$ & 1800 & 1 & 0.072 & 1 \\
\hline 2 & $\begin{array}{c}1 \mathrm{~mL} \\
\text { Syringe }\end{array}$ & 5000 & 1 & 0.072 & 1 \\
\hline 3 & $\begin{array}{c}5 \mathrm{~mL} \\
\text { Cartridge }\end{array}$ & 3000 & 5 & 0.064 & 5 \\
\hline
\end{tabular}




\section{Chapter 3}

Testing \& Validation

\subsection{Experimental Setup}

All experiments were conducted at Amgen's headquarters in Thousand Oaks, California with the help of the TAO team. Amgen TAO's proprietary technology called SpinCAM was used to capture the images of the high-speed spinning containers. A software application provides options for the number of images during and after spinning, the angular velocity in revolutions per minute, and saving the images as raw or processed images. Figures 3.1 and 3.2 detail the 3-D SpinCAM and Figure 3.3 shows an image of the SpinCAM with the container cover off showing the LED lights turned on.

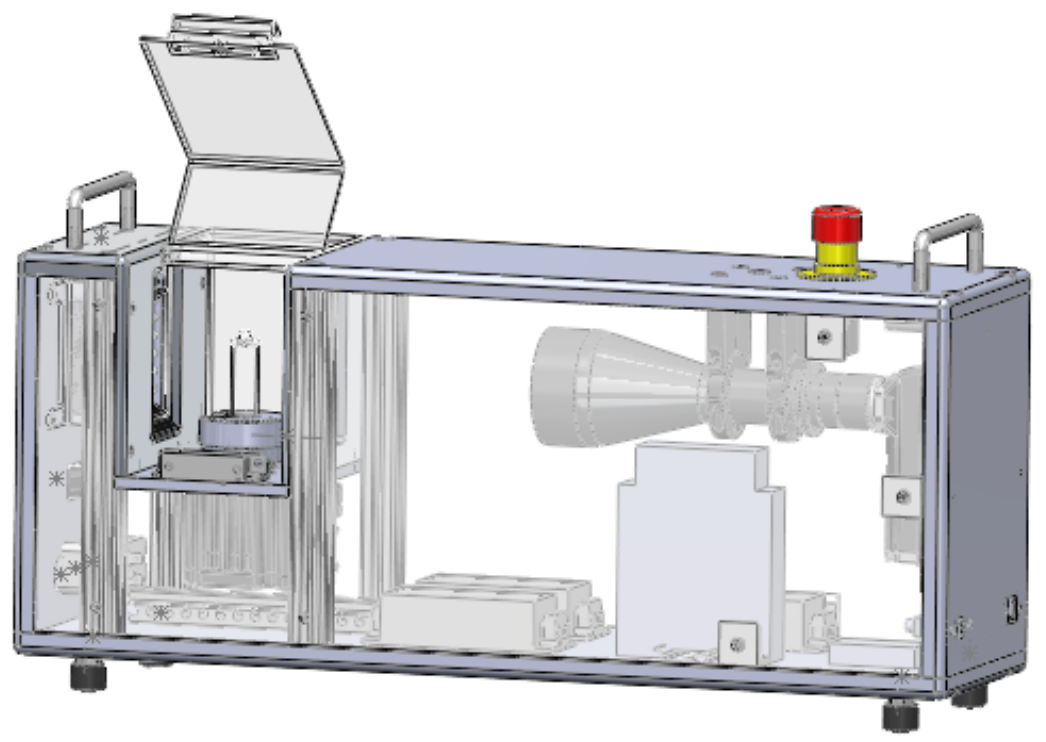

Figure 3.1 - A Solidworks rendering of the SpinCAM 


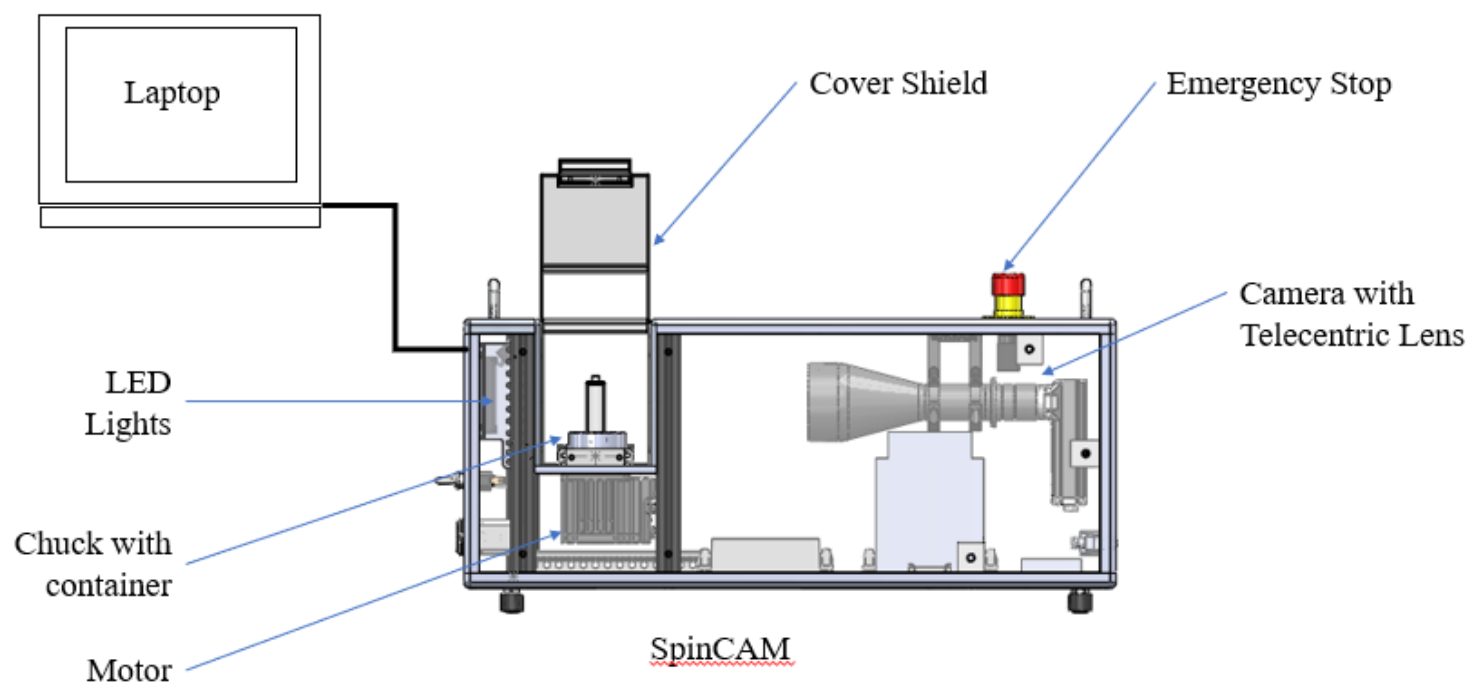

Figure 3.2 - A front view of SpinCAM with labeled key parts.

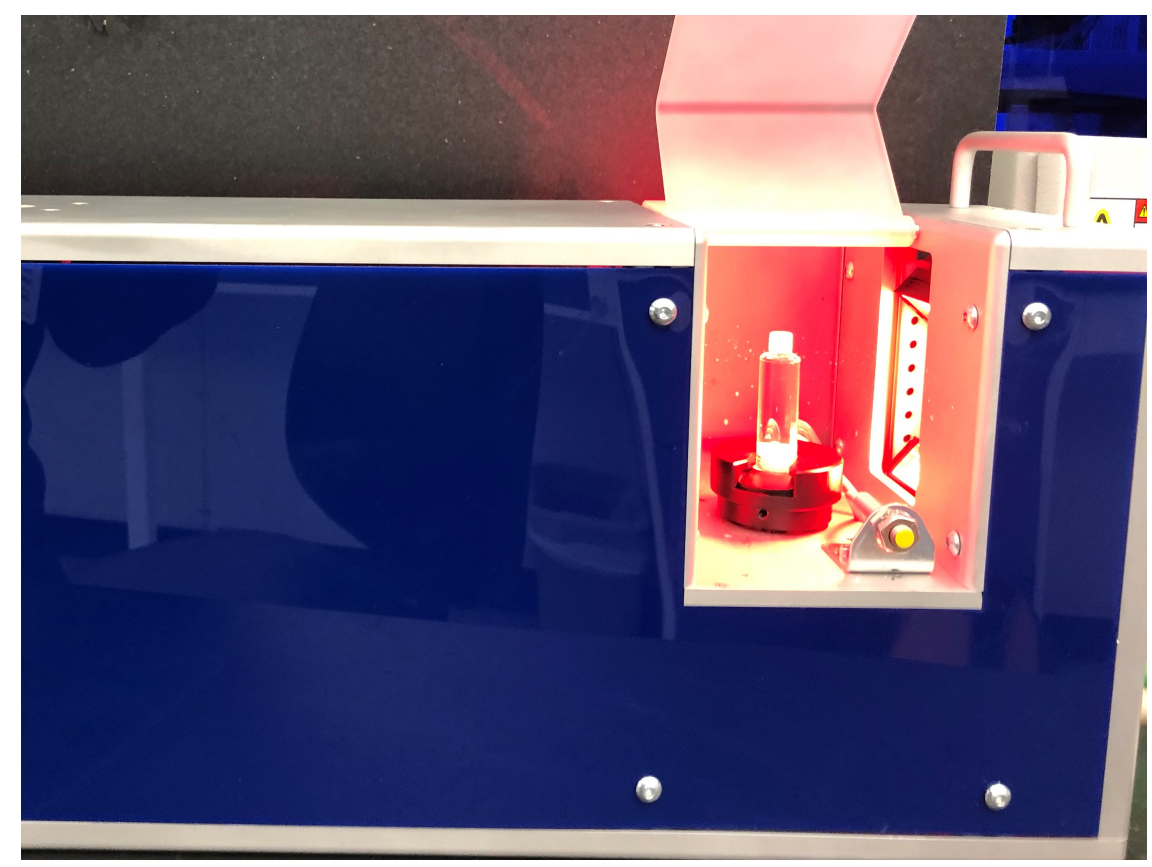

Figure 3.3 - A view of the spin CAM with the blue cover. 


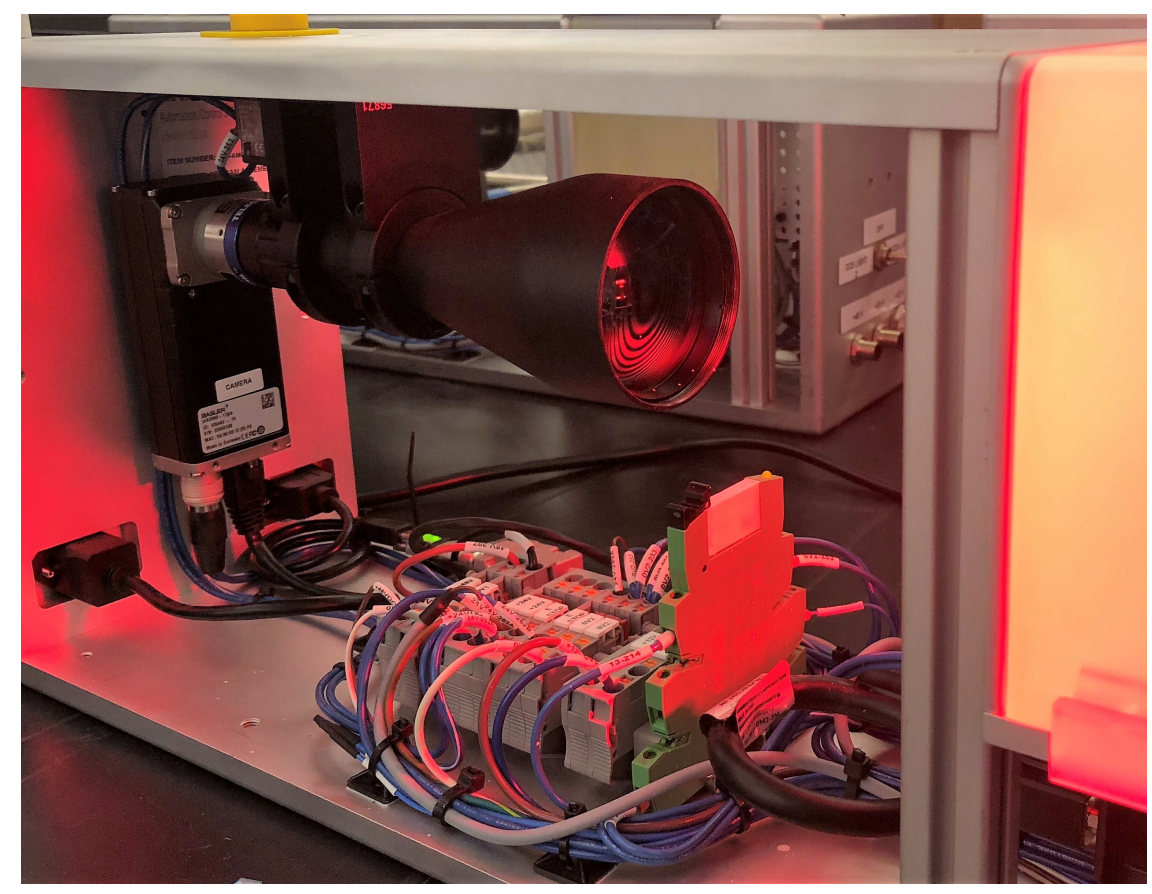

Figure 3.4 - A view of the SpinCAM showing a Basler camera with telephoto lens capturing images.

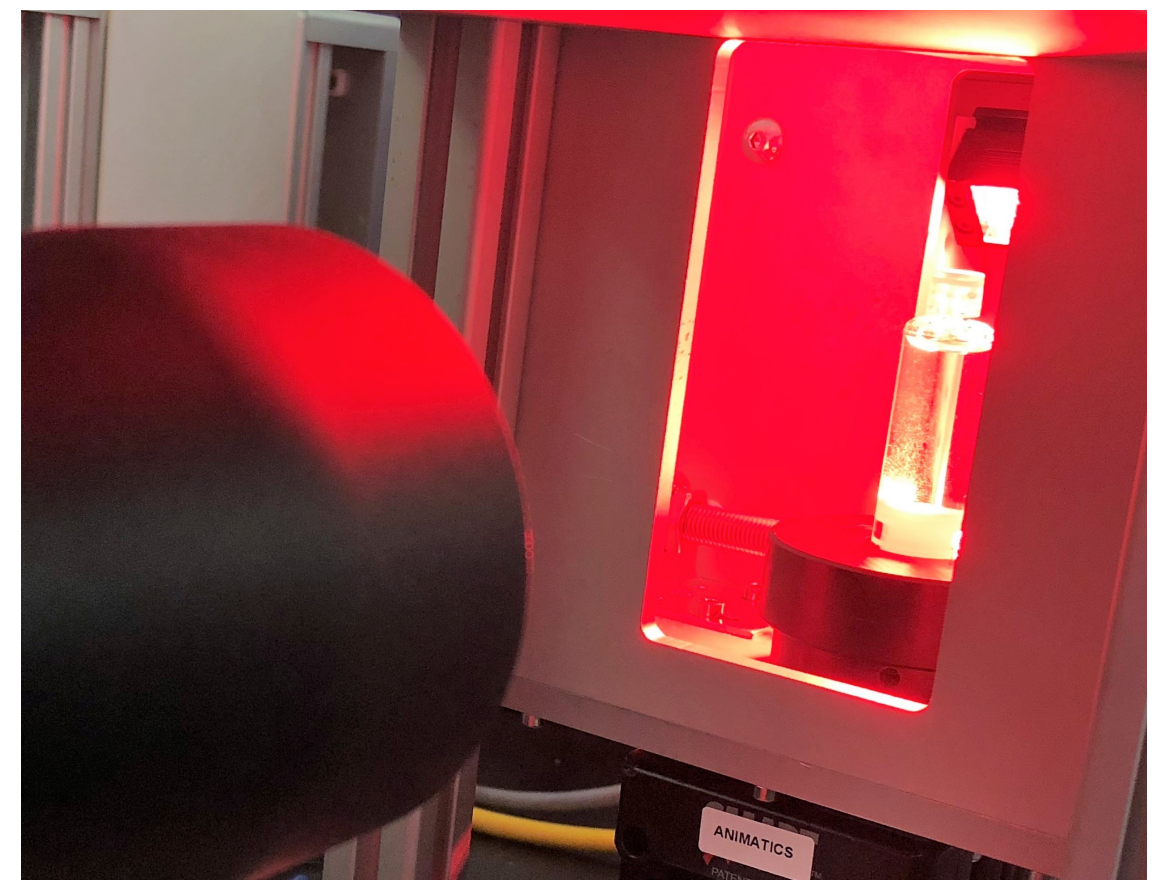

Figure 3.5 - A view of the camera facing the spinning cartridge with flashing LED lights. 


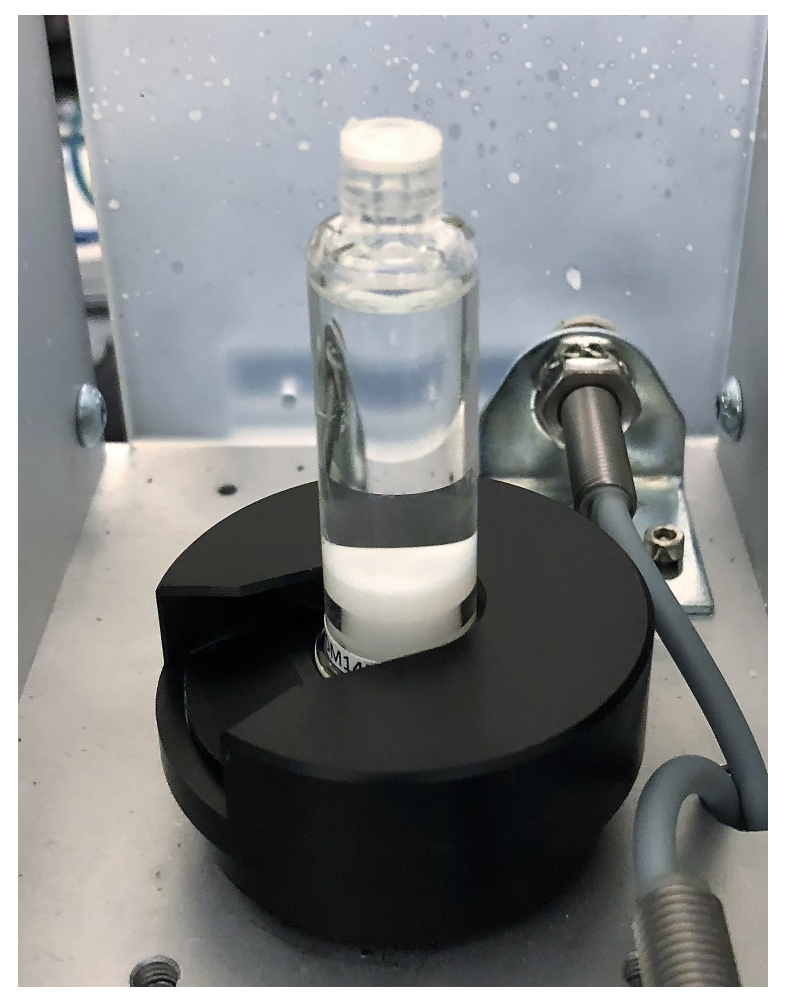

Figure 3.6 - $5 \mathrm{~mL}$ cartridge placed on the chuck inside the SpinCAM.

Figure 3.4 and 3.5 show the spinCAM operating during the spin-stop agitation. The LED lights turn on while the camera captures and processes the images. Figure 3.6 shows the cartridge securely inserted into the chuck. This chuck is designed for cartridge use only. When switching containers from a cartridge to a syringe, a modified chuck for the syringe is used as shown in Figure 3.7. The chuck is removed by unscrewing the hex fasteners with an Allen wrench. 


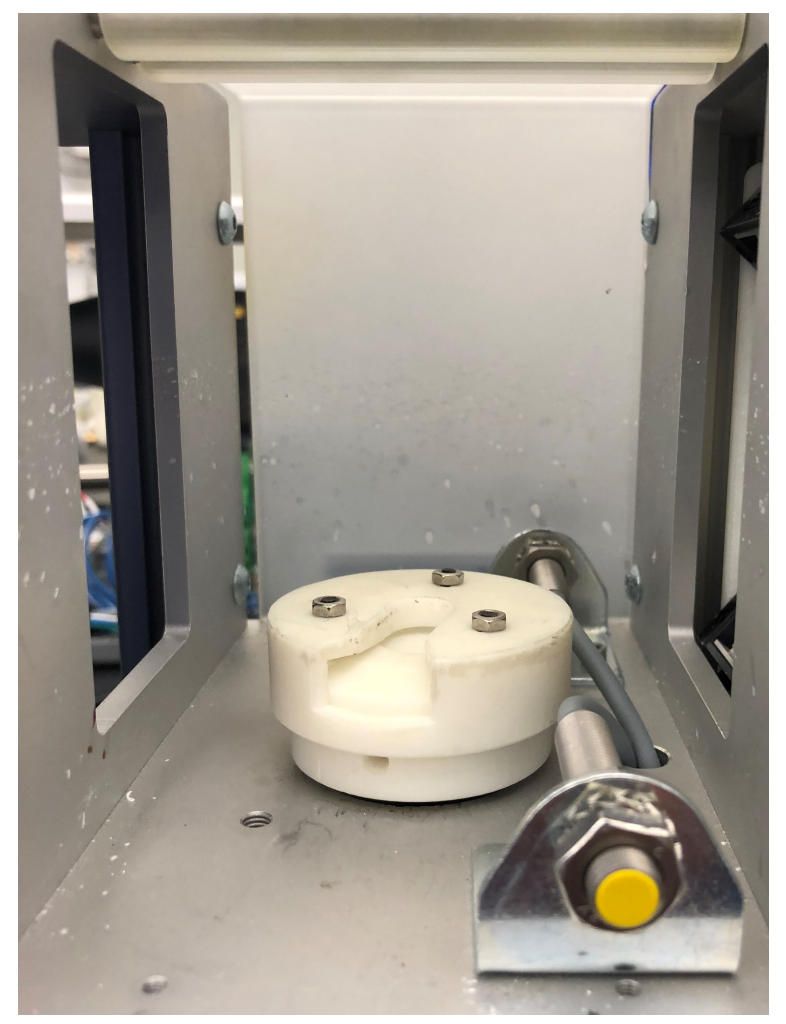

Figure 3.7 - A view of the syringe chuck inside the SpinCAM.

Numerous samples of $5 \mathrm{~mL}$ cartridges and $2.25 \mathrm{~mL}$ syringes were created. One sample of the $1 \mathrm{~mL}$ syringe was created for comparison with the validation models at $1800 \mathrm{rpm}$. The $5 \mathrm{~mL}$ cartridges were comprised of a dynamic viscosity of 1,5 and $10 \mathrm{cP}$. The fluid for the $2.25 \mathrm{~mL}$ syringes had a dynamic viscosity of 10 and $39 \mathrm{cP}$. All samples were injected with 15 or $25 \mu \mathrm{m}$ polystyrene beads using a micro pipette. The polystyrene beads were diluted with two drops in a beaker containing $10 \mathrm{~mL}$ of water to prevent over-saturation of beads in the liquid domain. This also provided better qualitative observation results.

The raw and processed images were extracted from a high speed camera (Basler Pilot piA2400-17gm). A telecentric lens (Edmund Optics 56675, 0.16X) is used for correct perspective and focus of the spinning container. Red LED arrays (CCS) are used to illuminate the container. All captured images can form a video clip to show 
the meniscus stretching to the bottom of the container as well as observe the particles swirling around the meniscus. The images can be further processed in MATLAB for image analysis to measure the approximate height of the meniscus.

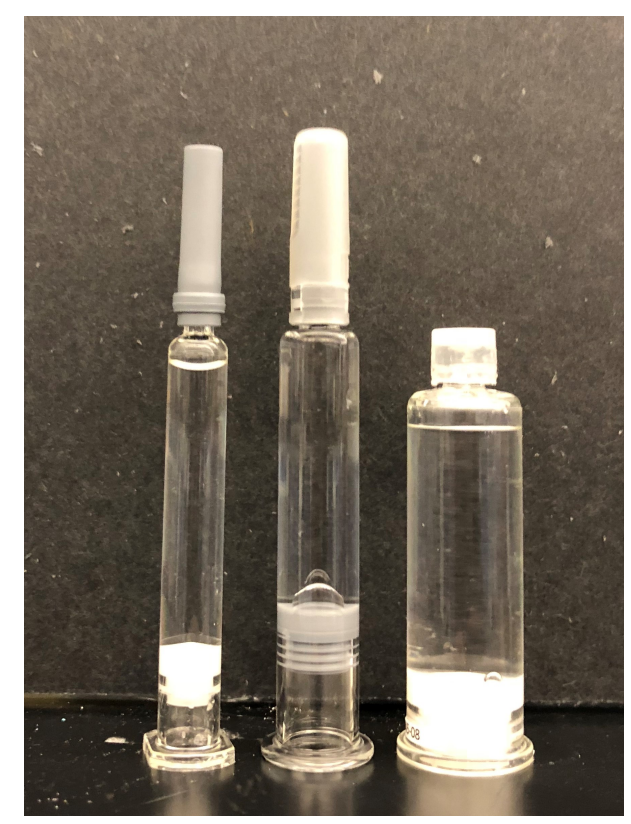

Figure 3.8 - A front view of the $1 \mathrm{~mL}$ syringe, $2.25 \mathrm{~mL}$ syringe, and $5 \mathrm{~mL}$ cartridge

The measured containers were a $1 \mathrm{~mL}$ syringe, $2.25 \mathrm{~mL}$ syringe, and a $5 \mathrm{~mL}$ cartridge as shown in Figure 3.8. Each container was verified for measurements with a digital caliper with a tolerance of \pm 0.005 as shown in Figure 3.9. The sample list with the measured air gaps and the dynamic viscosity are shown in the appendices.

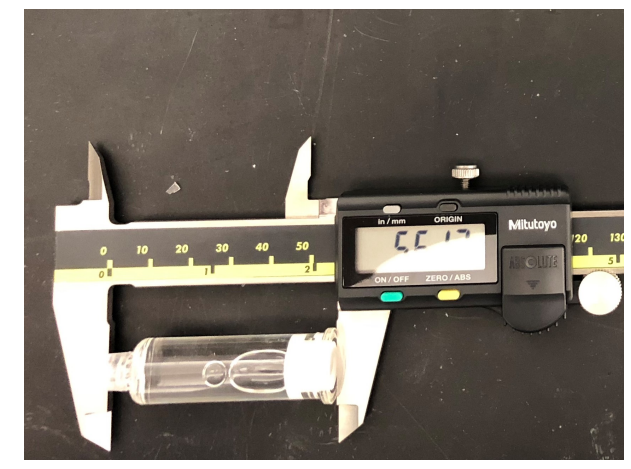

Figure 3.9 - An image of measuring the dimensions for the $5 \mathrm{~mL}$ cartridge 


\section{Chapter 4}

Results \& Discussion

This chapter is divided into sections of Amgen containers types of $1 \mathrm{~mL}$ syringe, 5 $\mathrm{mL}$ cartridge, and $2.25 \mathrm{~mL}$ syringe. Each container is composed of a combination of results detailing the Fluent and experiment relationship, 2-D Fluent cases with varying parameters, and 3-D vs 2-D Fluent cases.

\section{$4.11 \mathrm{~mL}$ Syringe}

\subsubsection{Results}

The first container is the validation model of a $1 \mathrm{~mL}$ glass syringe spinning at 1800 rpm with a $1 \mathrm{~mL}$ fill and an air gap of $8 \mathrm{~mm}$. The liquid solution was assumed as ideal water with a density of $1000 \mathrm{~kg} / \mathrm{m}^{3}$ and a surface tension of $0.072 \mathrm{~N} / \mathrm{m}$.

In this section, the $1 \mathrm{~mL}$ syringe compares the meniscus height in models made in experiments, 2-D, and 3-D. The meniscus height is used to show the Fluent and experiment cases show a quantitative agreement. The MATLAB meniscus height was $5.58 \mathrm{~mm}$, which was used to find the analytical solution using an iterative procedure from Lubarda [6] and described in chapter 2. The $1 \mathrm{~mL}$ syringe from the spinCAM is shown in Figure 4.1, which has pixel dimensions of a diameter of 202 and a height of 252 was used in MATLAB to find the proportionate meniscus height, which was $4.86 \mathrm{~mm}$. 


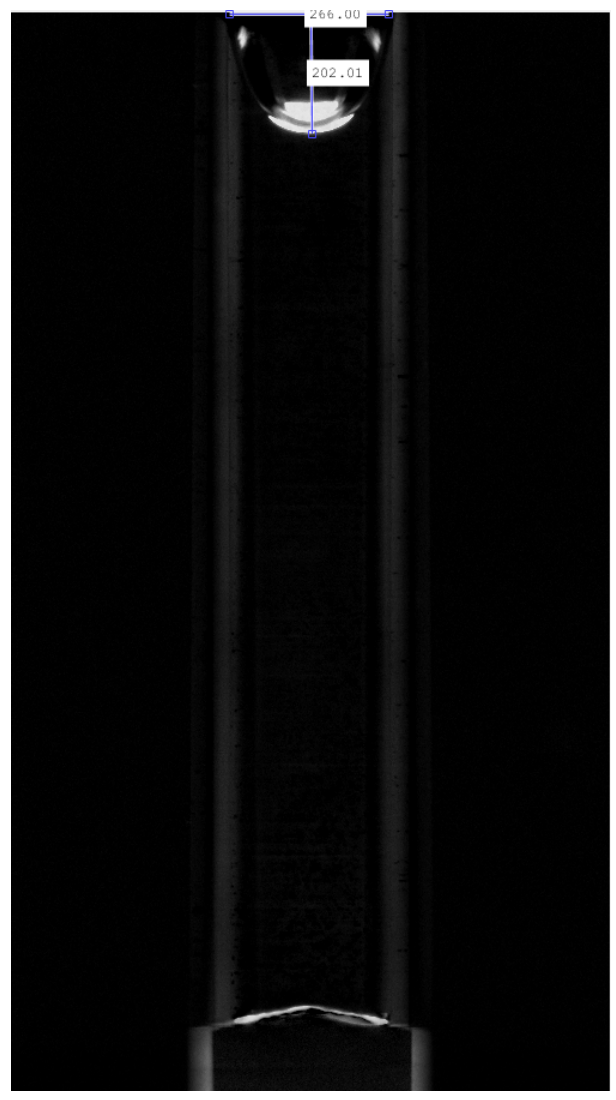

Figure 4.1 - An image of a $1800 \mathrm{rpm} 1 \mathrm{~mL}$ syringe at steady state with pixel dimensions in MATLAB.
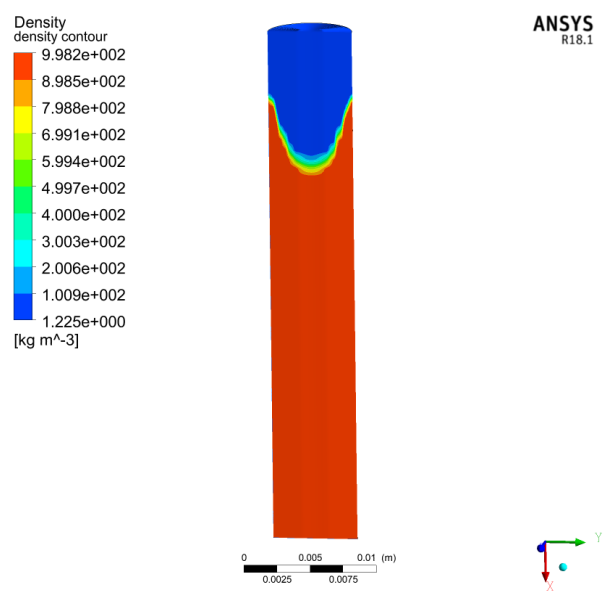

(a) A mid-section view of a density contour plot.
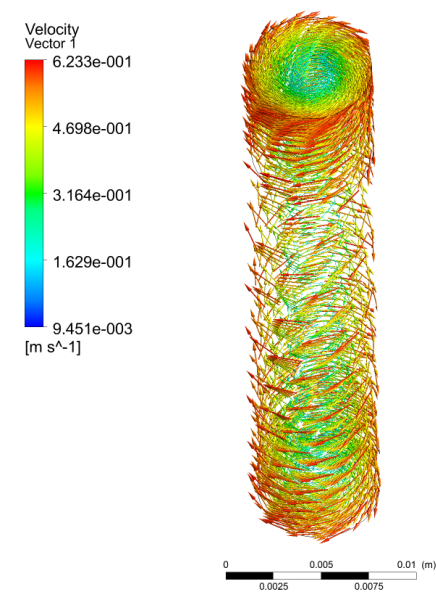

$\underset{\mathrm{R} 18.1}{\operatorname{ANSYS}}$

(b) A perspective view of the velocity vectors.

Figure 4.2 - 3-D models of a $1 \mathrm{~mL}$ syringe spinning at $1800 \mathrm{rpm}$ at 2.5 seconds. 
Figure 4.2 shows a mid-plane section view of a density contour plot and a velocity vector plot for a 3-D Fluent model. The meniscus height was found to be $5.24 \mathrm{~mm}$ using the image from Figure $4.2 \mathrm{a}$ in MATLAB. Figure $4.2 \mathrm{~b}$ shows a linear velocity profile where the highest velocity magnitude is $0.623 \mathrm{~m} / \mathrm{s}$ at near the walls and near zero at the axis of the flow.

In Table 4.1, all meniscus height values with their respective percent error are shown where the analytical (MATLAB) solution was $5.58 \mathrm{~mm}$, the 2D Fluent was $5.39 \mathrm{~mm}$ with a percent error of $3.40 \%$, the 3D Fluent was $5.24 \mathrm{~mm}$ with a percent error of $6.09 \%$ and the experimental solution had a height of $4.86 \mathrm{~mm}$ with a percent error of $12.90 \%$.

Table 4.1 - Meniscus height of a $1 \mathrm{~mL}$ syringe for Fluent, MATLAB, and experiment.

\begin{tabular}{|c|c|c|}
\hline Solution Type & Meniscus Height (mm) & Percent Error (\%) \\
\hline \hline MATLAB & 5.58 & - \\
\hline 2D Fluent & 5.39 & 3.40 \\
\hline 3D Fluent & 5.24 & 6.09 \\
\hline Experimental & 4.86 & 12.90 \\
\hline
\end{tabular}

For a $1 \mathrm{~mL}$ syringe with a $1 \mathrm{~mL}$ fill of water is spun at $5000 \mathrm{rpm}$. Figure 4.3 shows a density contour plot and a velocity vector plot at $t=2.5$ seconds when it is at solid rigid motion. Figure 4.3a shows the increase in angular velocity stretched the meniscus to the bottom of the container. In Figure 4.3b, the velocity vectors show a uniformly increasing velocity at the radial position from the axis to the wall of the container. It is expected that the highest velocity is at $1.674 \mathrm{~m} / \mathrm{s}$ for an angular velocity of $5000 \mathrm{rpm}$. 

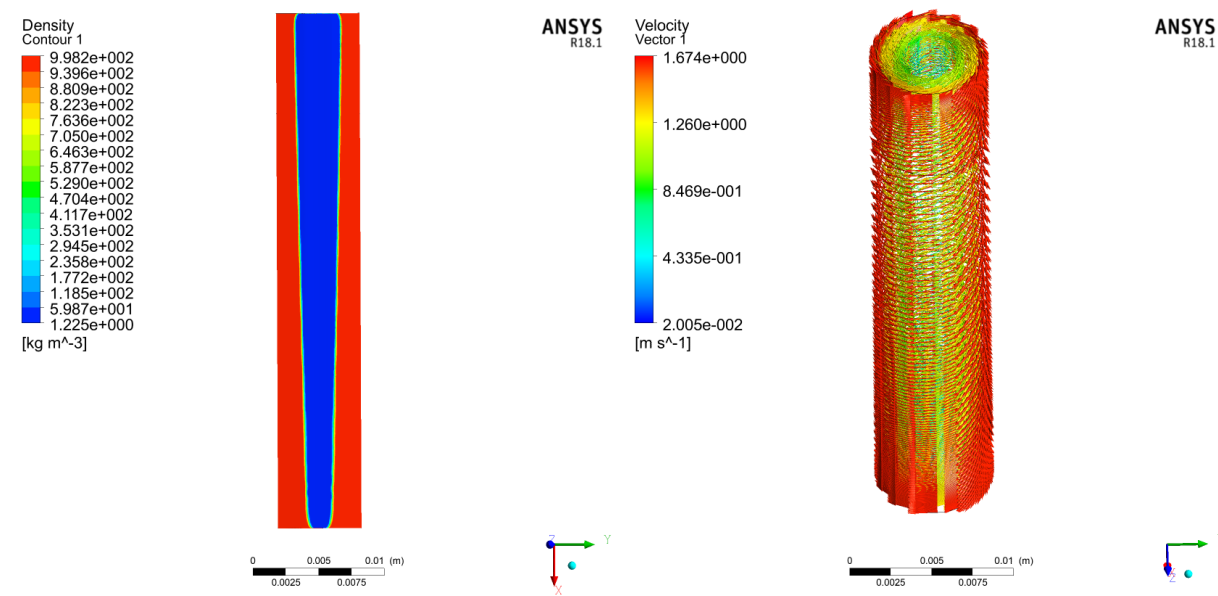

(a) Density Contour Plot

(b) Velocity Vector Plot

Figure 4.3 - Plots for a $1 \mathrm{~mL}$ filled $1 \mathrm{~mL}$ syringe at $5000 \mathrm{rpm}$.

In Fluent, streamlines are assumed to be steady state flows even during transient simulations and calculated using a Runge-Kutta method of vector variable integration [29]. Figure 4.4 shows the streamlines at the top portion of the container where the air-liquid interaction is most dominant. The streamlines tangent to the velocity are highest at approximately $1.674 \mathrm{~m} / \mathrm{s}$ in the outer radii or near the wall of the container. The streamlines visualize the flow of the gas-liquid interactions as decreasing flows from near the wall to the axis of the container. Since this is occurring during steady state, the pathlines, streaklines, and streamlines are the same trajectory. Figure 4.4 displays 100 uniform streamline points created in ANSYS CFD-post. Due to the mesh cell size and 3-D case, additional streamline points requires more computational time. 

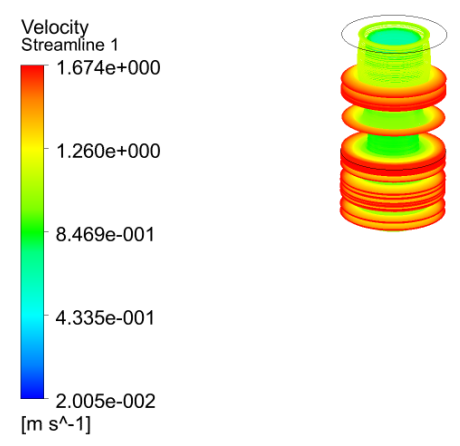

ANSYS

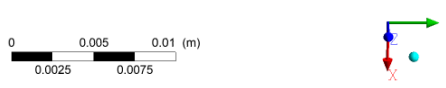

Figure 4.4 - A streamline plot during steady state at 2.5 seconds for a $1 \mathrm{~mL}$ syringe spinning at $5000 \mathrm{rpm}$. 

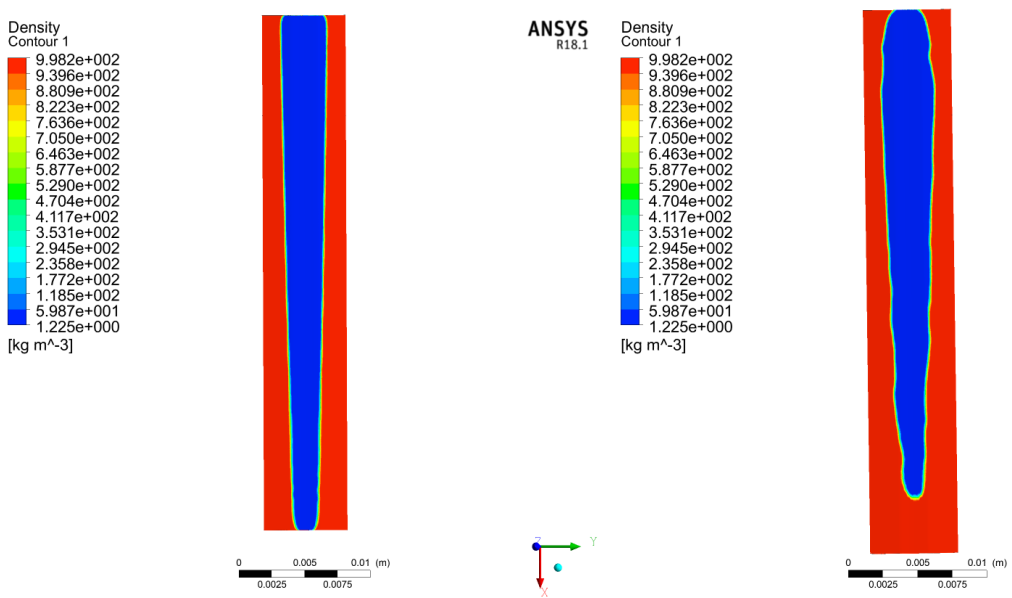

ANSYS

(a) $\mathrm{t}=2.50$ seconds

(b) $t=2.60$ seconds
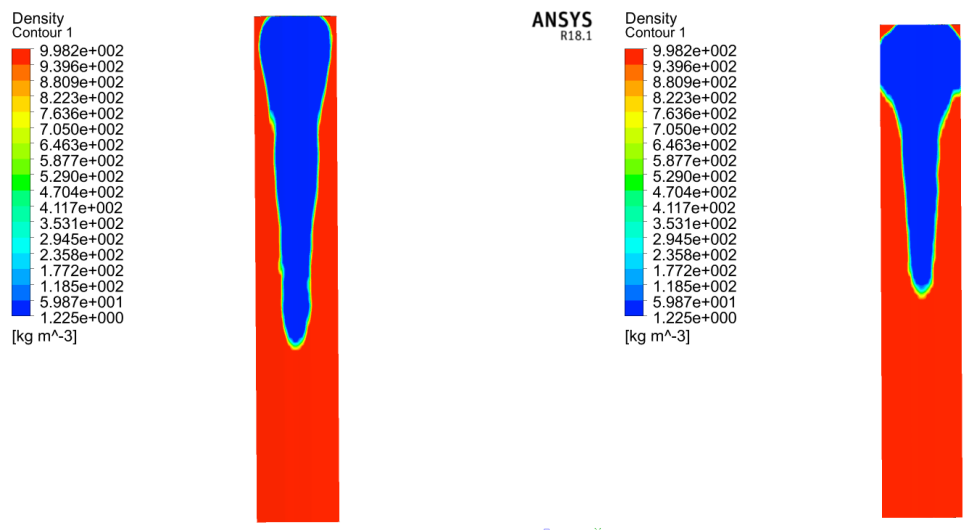

$\underset{\text { R18.1 }}{\text { ANSYS }}$
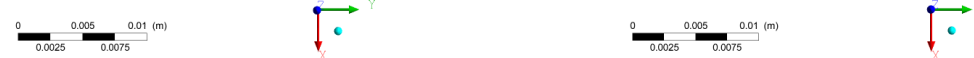

(c) $\mathrm{t}=2.70$ seconds

(d) $t=2.73$ seconds
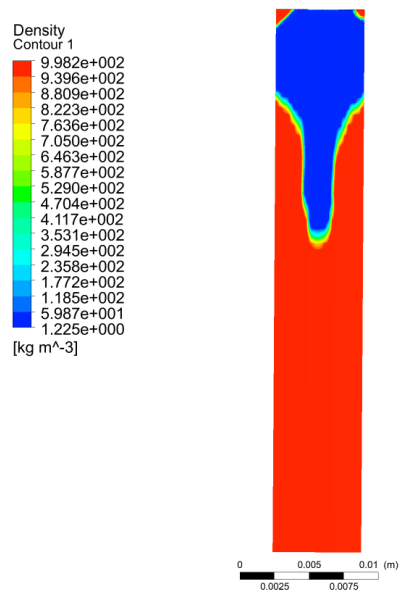

$\underset{\text { R18.1 }}{\text { ANSYS }}$

(e) $\mathrm{t}=2.75$ seconds

Figure 4.5 - A sectional density contour plot for a $1 \mathrm{~mL}$ syringe spinning down to rest from 2.5 to 2.75 seconds. 


\subsubsection{Discussion}

Based on Table 4.1, errors in the 2-D and 3-D model can be mostly attributed to spatial discretization or mesh error and iteration error. The mesh and iteration error occur because a finer mesh can lead to more accurate results and iteration error can be attributed to the time step size compounding the error.

There is a higher percent error for the experiment because the samples were not made in a clean room environment where fibers and micro bubbles can be present in the samples. Also, the camera could not fully capture the top of the syringe because the container was too long to capture. Error can be attributed to the measurement and uncertainty of the meniscus height for the experiment. Additional errors in include the geometry of the containers. Since both Fluent and the analytical model assume a perfectly shaped cylinder, the experiment had some error because of the geometry of the syringe not being a perfectly shaped cylinder.

The $1 \mathrm{~mL}$ syringe spinning at $5000 \mathrm{rpm}$ shows dwetting at the bottom with an increase in angular velocity. Furthermore, Figure 4.5 closely examines the instabilities during spin-down as not symmetric for a 3-D model. A closer examination of spindown will be discussed in the $5 \mathrm{~mL}$ cartridges. 


\section{$4.25 \mathrm{~mL}$ Cartridge}

\subsubsection{Results}

This section details a $5 \mathrm{~mL}$ cartridge spinnng at $3000 \mathrm{rpm}$ for meniscus height verification with the experiment, varying the dynamic viscosity, and showing the difference between 2-D and 3-D Fluent models.

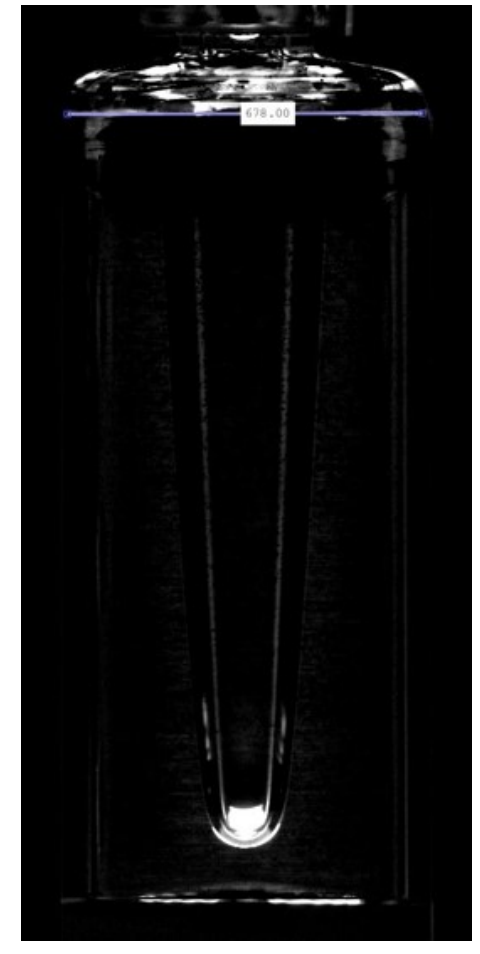

(a) Width Measurement

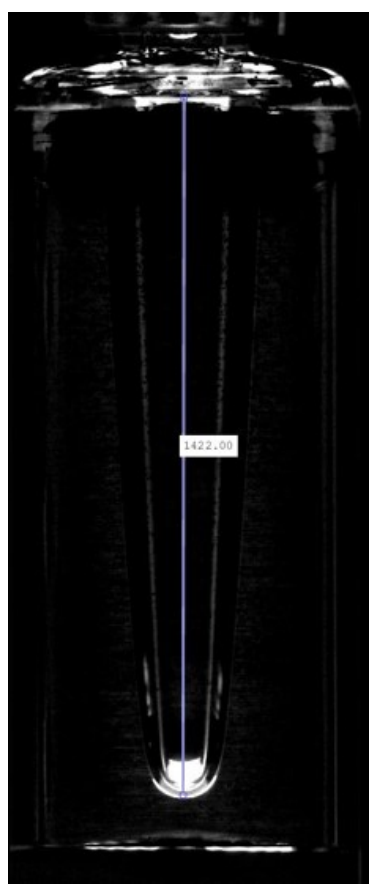

(b) Height Measurement

Figure 4.6 - $5 \mathrm{~mL}$ cartridge spinning at $3000 \mathrm{rpm}$ using pixel measurements

Figure 4.6 shows images from the experiment with pixel measurements when the $5 \mathrm{~mL}$ cartridge is at steady state at approximately 1.5 seconds. The width measurement of 670 pixels in Figure 4.6a provides a proportional value to the inner diameter of the container to calculate the meniscus height of 1422 pixels as shown in Figure 4.6b. 
Table 4.2 detail the meniscus height for the different solution types where the experiment had $30.67 \mathrm{~mm}$, the 2-D model had $31.28 \mathrm{~mm}$ with a percent difference of $1.97 \%$, and the 3-D model had $28.89 \mathrm{~mm}$ with a percent difference of $5.98 \%$.

Table 4.2 - Meniscus Height of a $5 \mathrm{~mL}$ Cartridge at $3000 \mathrm{rpm}$ for Fluent and Experiment.

\begin{tabular}{|c|c|c|}
\hline Solution Type & Meniscus Height $\mathbf{( m m})$ & Percent Difference (\%) \\
\hline \hline Experimental & 30.67 & - \\
\hline 2-D Fluent & 31.28 & 1.97 \\
\hline 3-D Fluent & 28.89 & 5.98 \\
\hline
\end{tabular}

Figure 4.7 shows a $5 \mathrm{~mL}$ cartridge spinning $3000 \mathrm{rpm}$ to rest from approximately 1.50 seconds to 1.80 seconds. These are approximate time steps based on images from the 2-D Fluent images and based on the approximate time interval between each frame. A comparison can be made with the 2-D Fluent images on the density plot in Figure 4.8, which also shows a bubble separating from meniscus and splashing to the top of the container.

Similar to Figure 4.7, a time capture of spin-down to rest for the density contour plot from 1.50 to 1.90 seconds is shown in Figure 4.8. At 1.75 seconds, a bubble separates from the meniscus and launches upward to create a residue at the top of the container. Figure 4.9 shows the streamlines during the same time frame. The streamlines are shown as $\mathrm{kg} / \mathrm{s}$ since the streamlines are multiplied by the density to get the mass flow rate. 


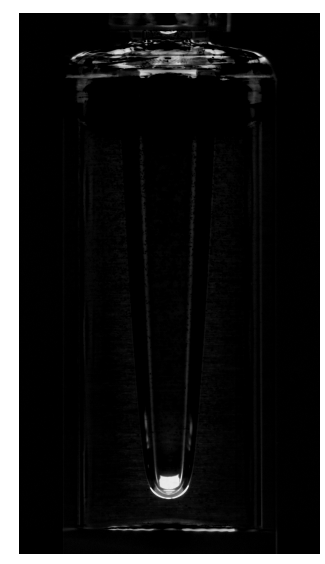

(a) $t \approx 1.50$ seconds

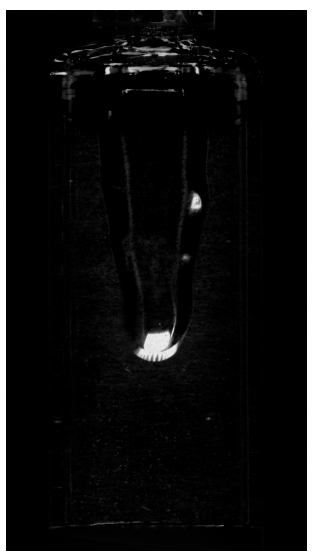

(c) $\mathrm{t} \approx 1.65$ seconds

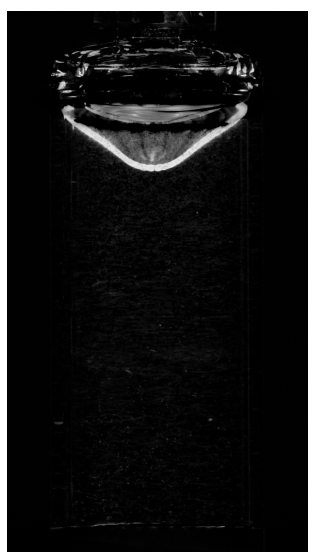

(e) $\mathrm{t} \approx 1.75$ seconds

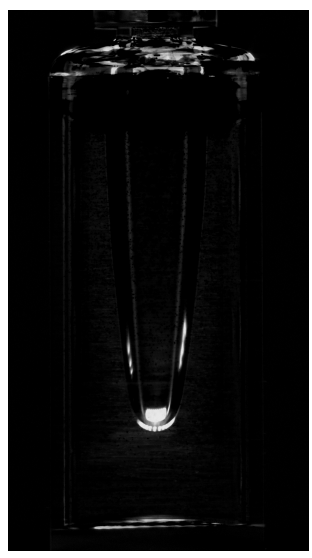

(b) $\mathrm{t} \approx 1.60$ seconds

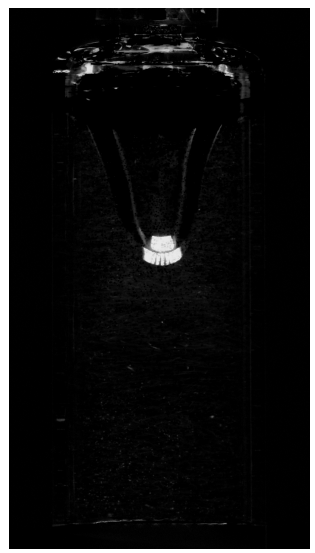

(d) $\mathrm{t} \approx 1.70$ seconds

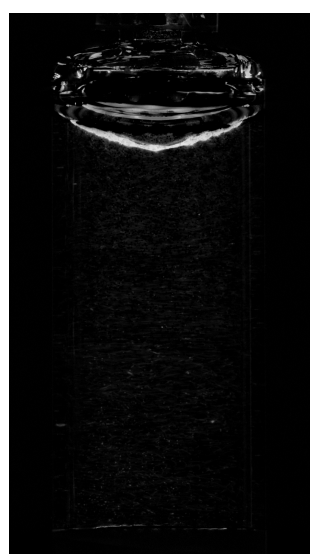

(f) $\mathrm{t} \approx 1.80$ seconds

Figure 4.7 - Captured images from the SpinCAM for a $5 \mathrm{~mL}$ cartridge spinning at $3000 \mathrm{rpm}$. 


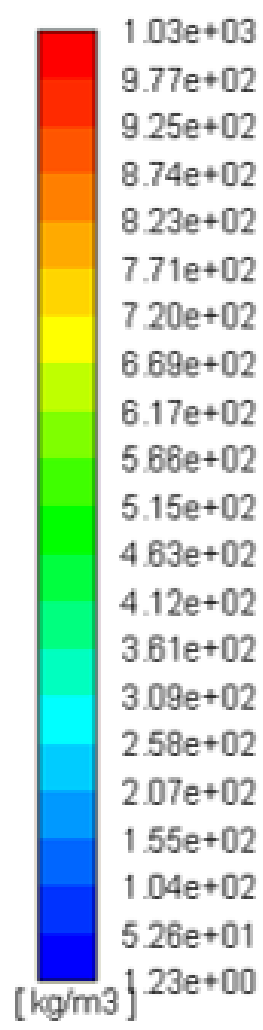

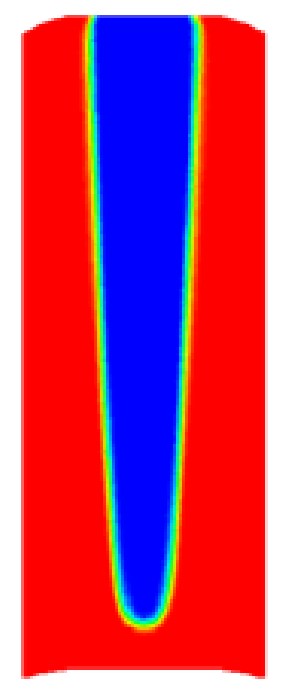

(a) $\mathrm{t}=1.50 \mathrm{~s}$

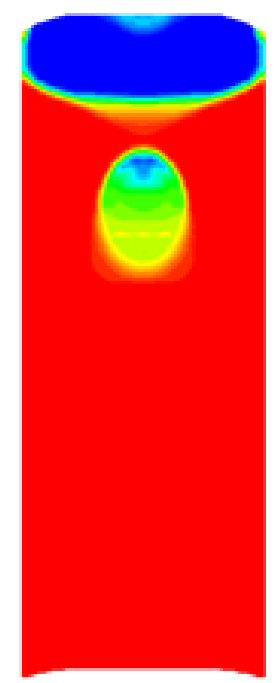

(d) $\mathrm{t}=1.75 \mathrm{~s}$

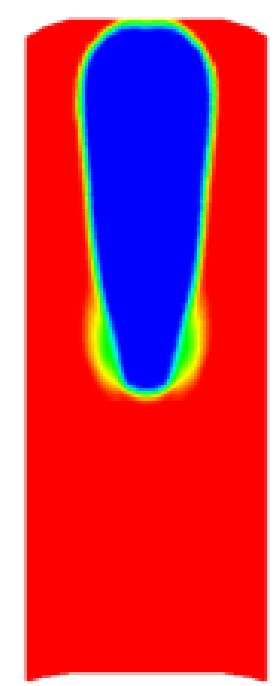

(b) $\mathrm{t}=1.60 \mathrm{~s}$

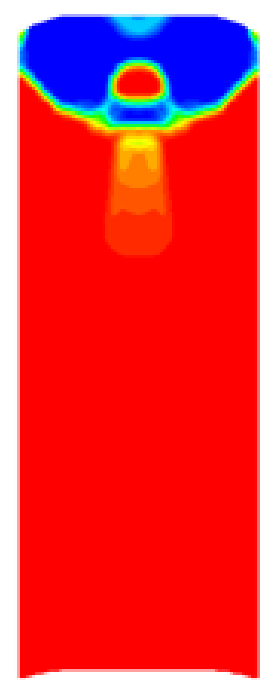

(e) $\mathrm{t}=1.80 \mathrm{~s}$

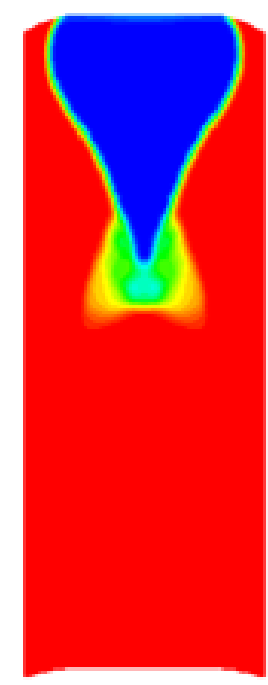

(c) $\mathrm{t}=1.65 \mathrm{~s}$

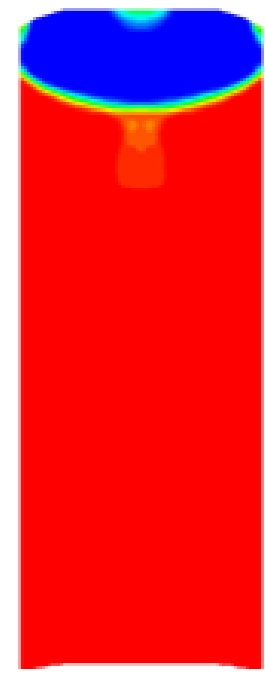

(f) $\mathrm{t}=1.90 \mathrm{~s}$

Figure 4.8 - Density contour plots from Fluent for a $5 \mathrm{~mL}$ cartridge spinning at $3000 \mathrm{rpm}$ to rest. 


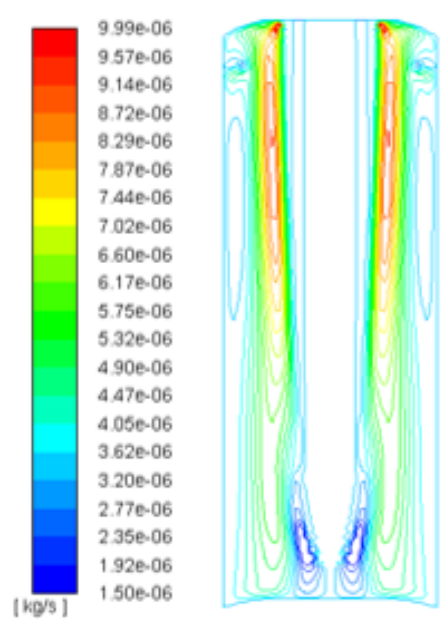

(a) $\mathrm{t}=1.50 \mathrm{~s}$
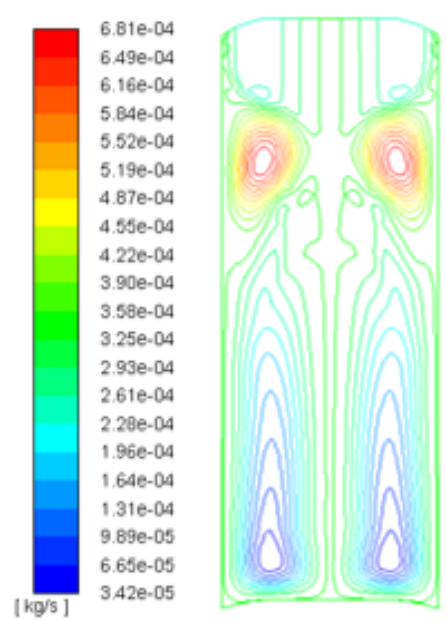

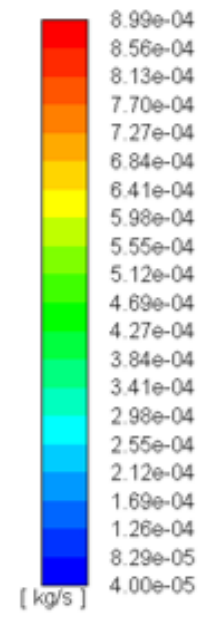

(d) $\mathrm{t}=1.75 \mathrm{~s}$

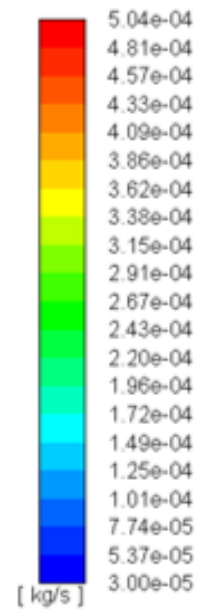

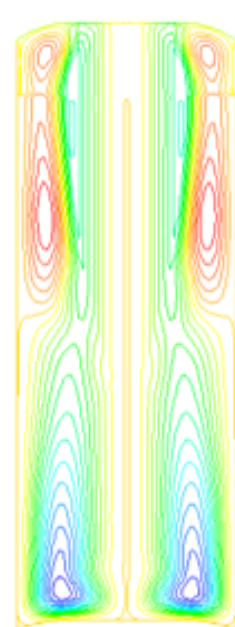

(b) $\mathrm{t}=1.60 \mathrm{~s}$

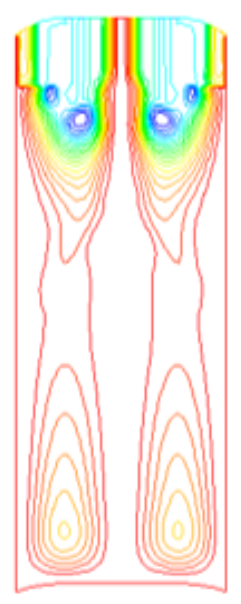

(e) $\mathrm{t}=1.80 \mathrm{~s}$
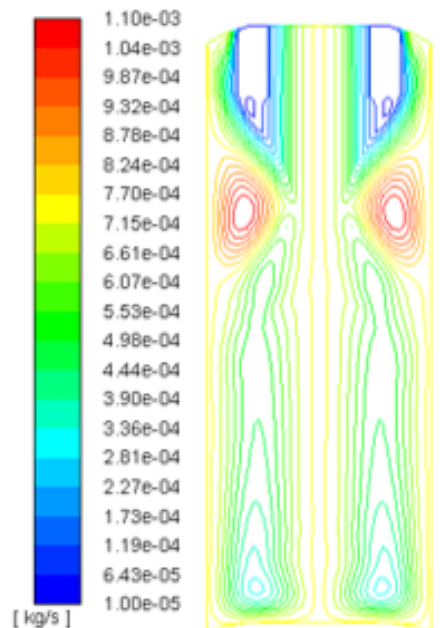

(c) $\mathrm{t}=1.65 \mathrm{~s}$
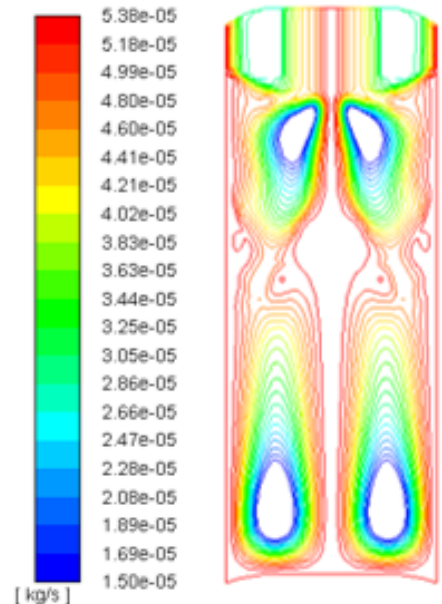

(f) $\mathrm{t}=1.90 \mathrm{~s}$ 0.02 (in)

Figure 4.9 - Streamline contour plot from Fluent for a $5 \mathrm{~mL}$ cartridge spinning at $3000 \mathrm{rpm}$ to rest. 


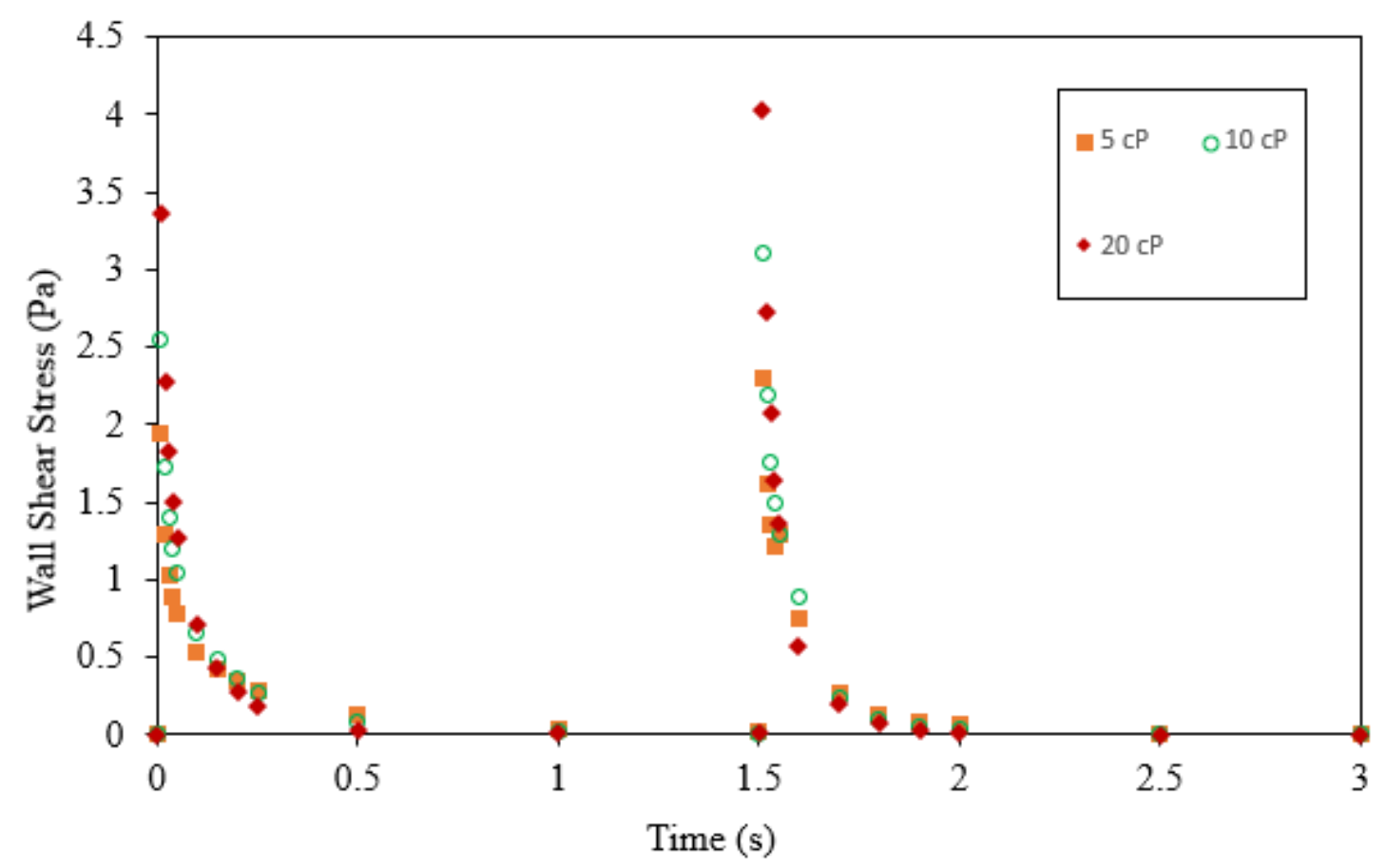

Figure 4.10 - Wall shear stress for dynamic viscosity of 5, 10, and $20 \mathrm{cP}$.

For varying viscosity parameters of 5,10 , and $20 \mathrm{cP}$, the wall shear stress across time is shown in Figure 4.10. The $20 \mathrm{cP}$ container has the highest wall shear stress which makes sense since a higher dynamic viscosity creates a higher shear stress. The wall shear stress also increases during the spin-down due to the abrupt centripetal force.

Figure 4.11 shows how the average velocity for the container reaches steady state for varying the dynamic viscosity. At $20 \mathrm{cP}$, the container reaches steady state earlier the 10 and $5 \mathrm{cP}$ containers. This makes sense since the Reynold number and the steady state time used by Greenspan [3] is the lowest. For the $5 \mathrm{cP}$ container, it reaches steady state the slowest. 


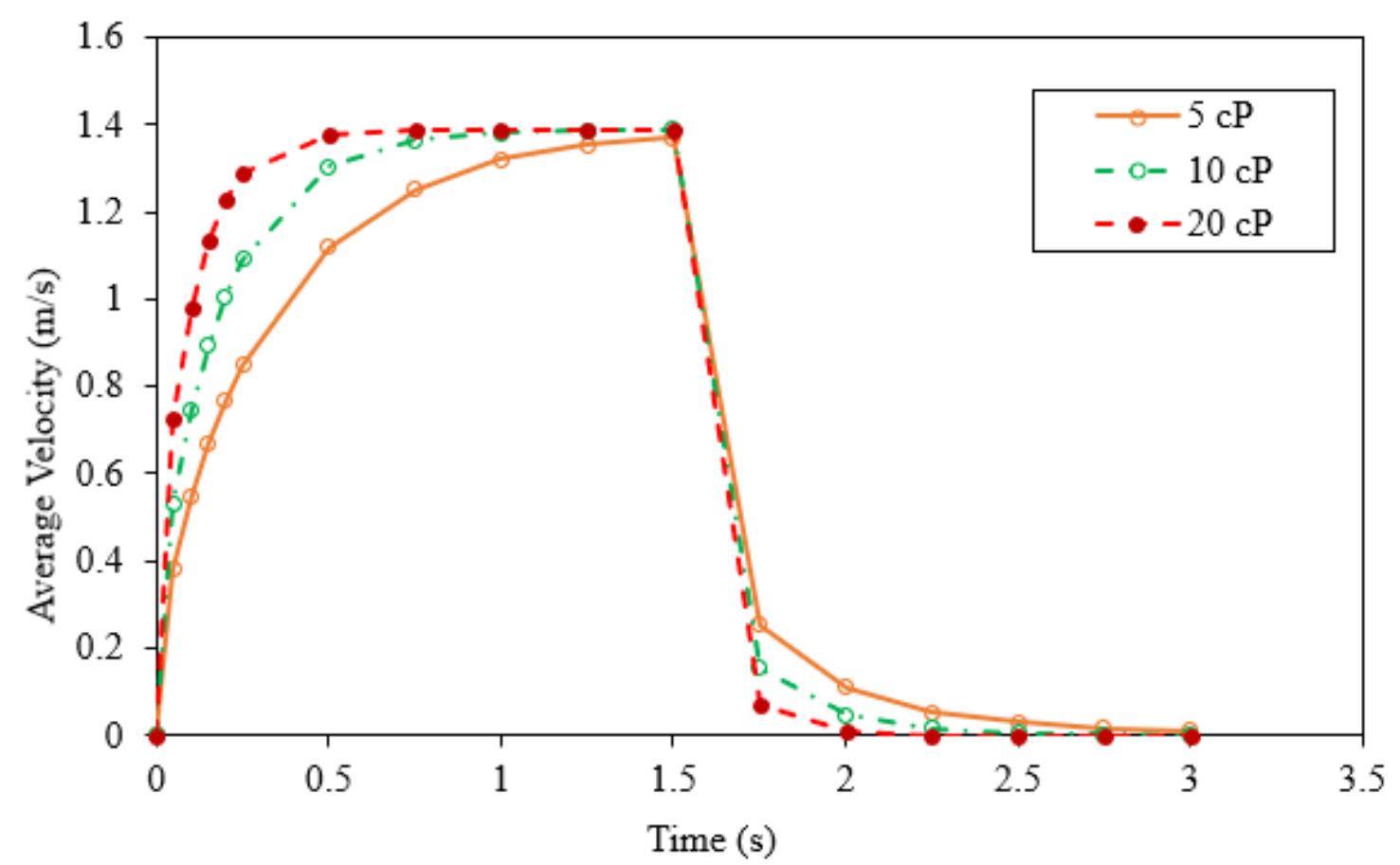

Figure 4.11 - Average Angular Velocity for dynamic viscosity of 5, 10, and $20 \mathrm{cP}$.

Figure 4.12 shows the differences in the dynamic viscosity containers when time is 3.0 seconds. At $5 \mathrm{cP}$, there is no residue at the top of the container as shown in Figure 4.12a. At $10 \mathrm{cP}$, there is a small bubble at the top as shown in Figure 4.12b. Finally, for $20 \mathrm{cP}$, there is a big bubble or splash as shown in Figure 4.12c. 


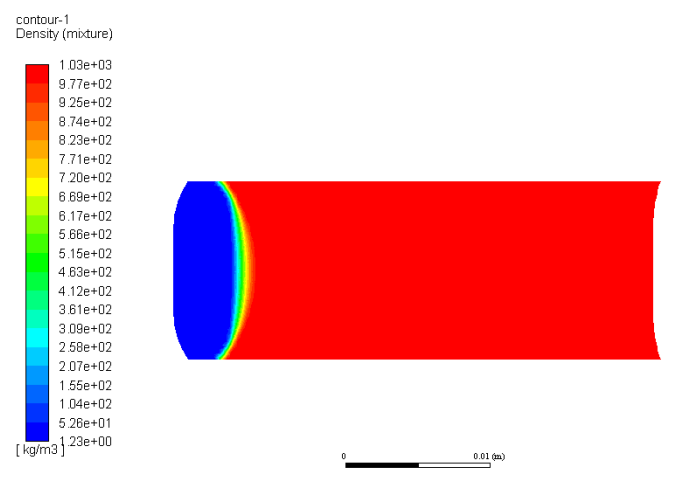

(a) $5 \mathrm{cP}$
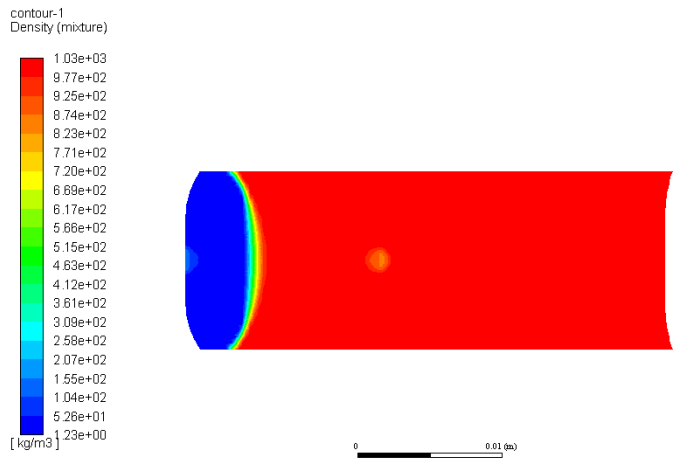

(b) $10 \mathrm{cP}$
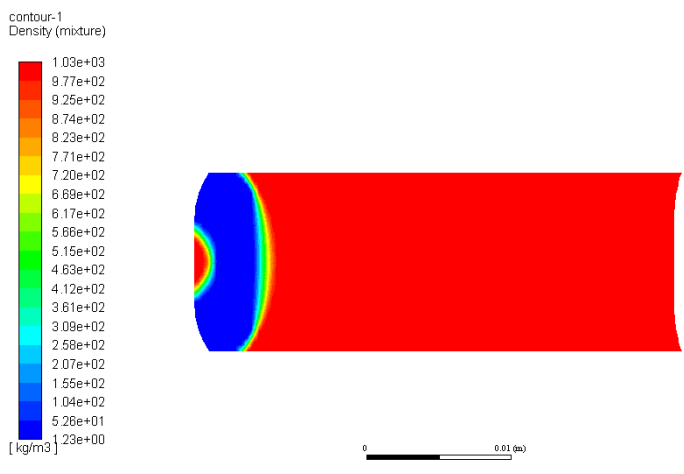

(c) $20 \mathrm{cP}$

Figure 4.12 - Density contour plot comparison at $t=3.0$ seconds

To take a closer look at the $5 \mathrm{~mL}$ cartridge at $3000 \mathrm{rpm}$ with a dynamic viscosity of $20 \mathrm{cP}$, a 3-D Fluent study is shown in Figure 4.13 at 1.5 seconds during steady state. Figure 4.14a shows a velocity vector plot at a perspective view and Figure 
4.14b shows a contour streamline plot of uniform distribution for 30 particle points. Figure $4.14 \mathrm{c}$ shows a perspective of the density contour plot. Figure 4.14 shows a time capture from spin-down to rest for a sectional density contour plot. Furthermore, Figure $4.14 \mathrm{f}$ shows a full 3 - $\mathrm{D}$ view at a final state at $\mathrm{t}=3.0$ seconds. There are water droplets at the top as well as residue at the top of the cylinder due to the instabilities during spin-down.
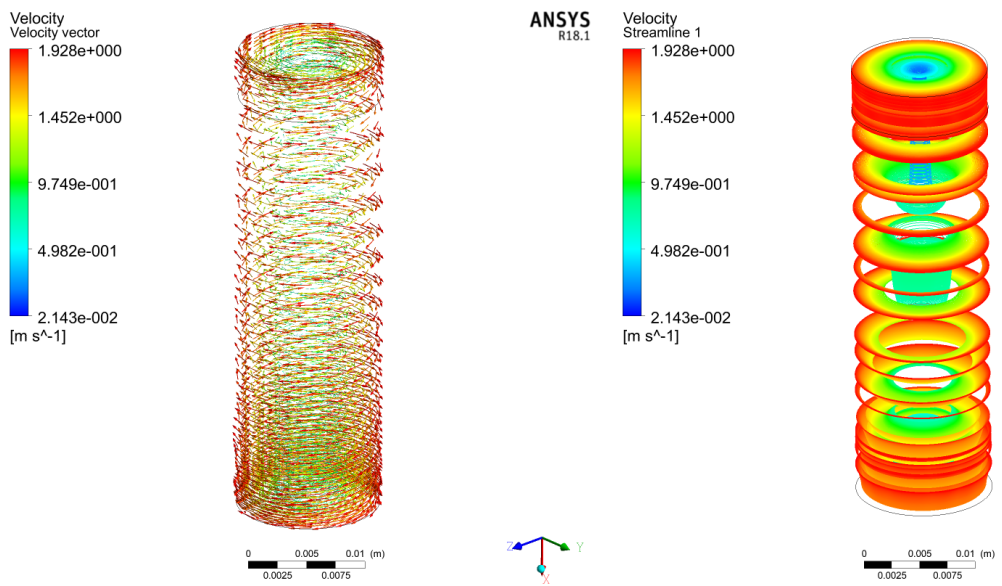

$\underset{\text { R18.1 }}{\operatorname{ANSYS}}$
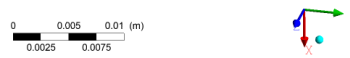

(a) Velocity Vectors Plot

(b) Contour Streamline Plot
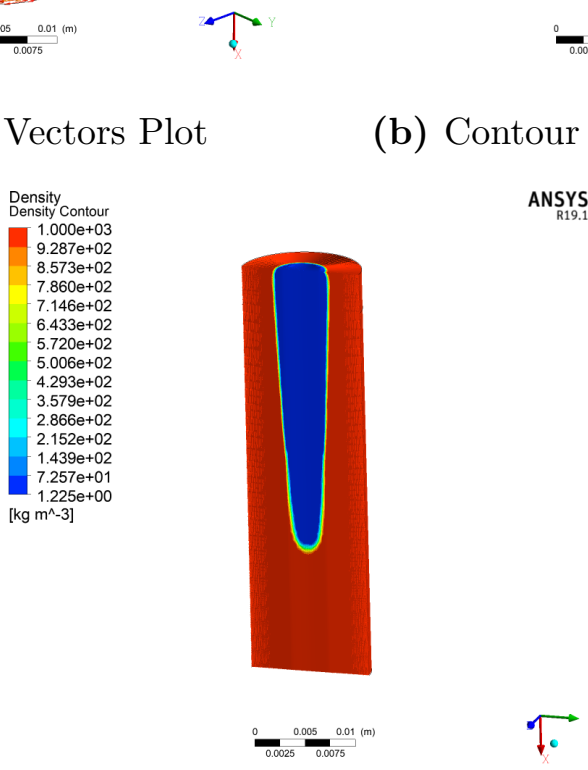

(c) Density Contour Plot

Figure 4.13 - A sectional density contour plot for a $5 \mathrm{~mL}$ cartridge spinning at 3000 rpm at 1.5 seconds. 

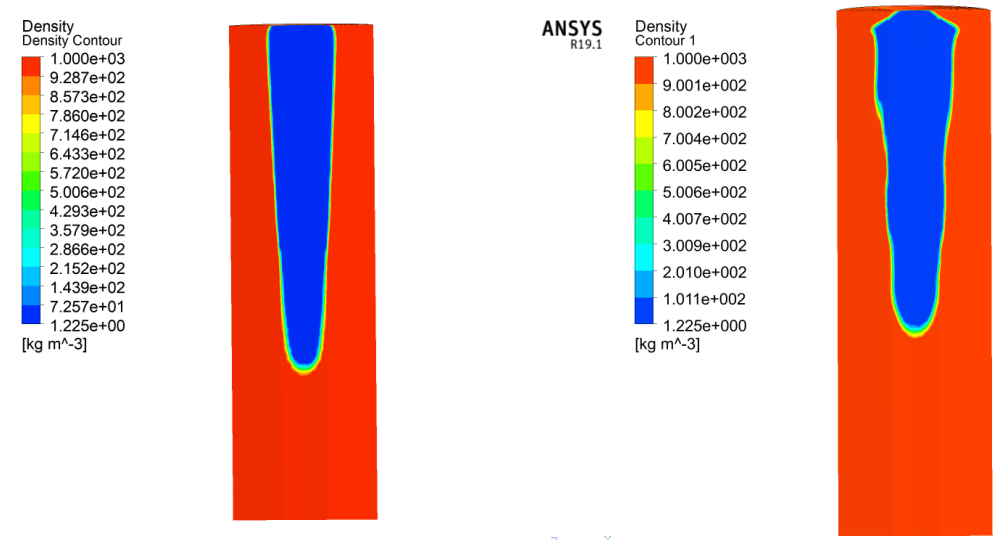

ANSYS
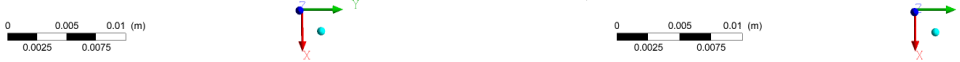

(a) $\mathrm{t}=1.50$ seconds

(b) $\mathrm{t}=1.60$ seconds
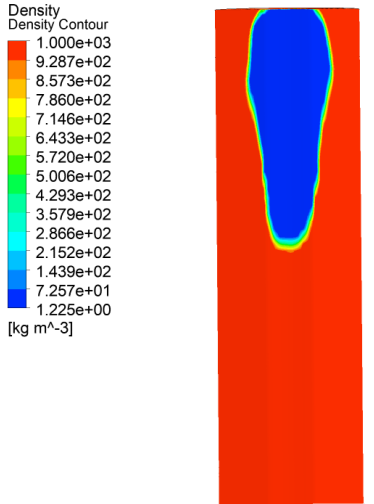

ANSYS
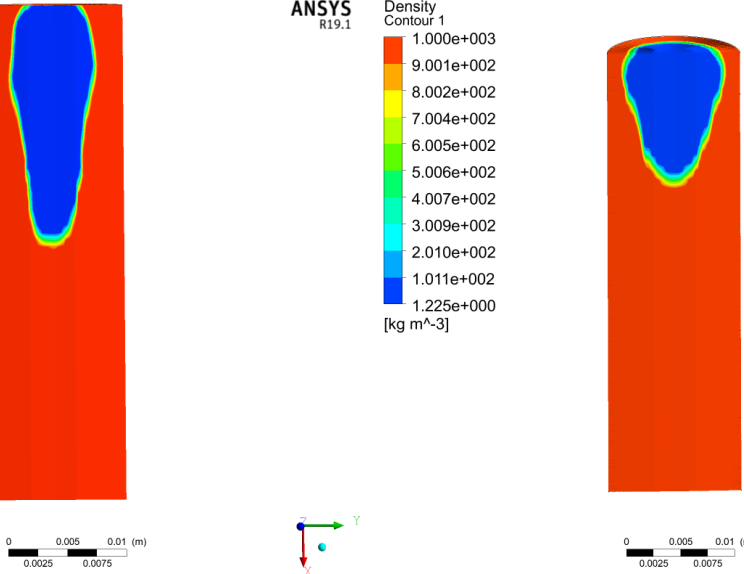

ANSYS
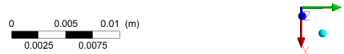

(c) $\mathrm{t}=1.70$ seconds

(d) $\mathrm{t}=1.80$ seconds
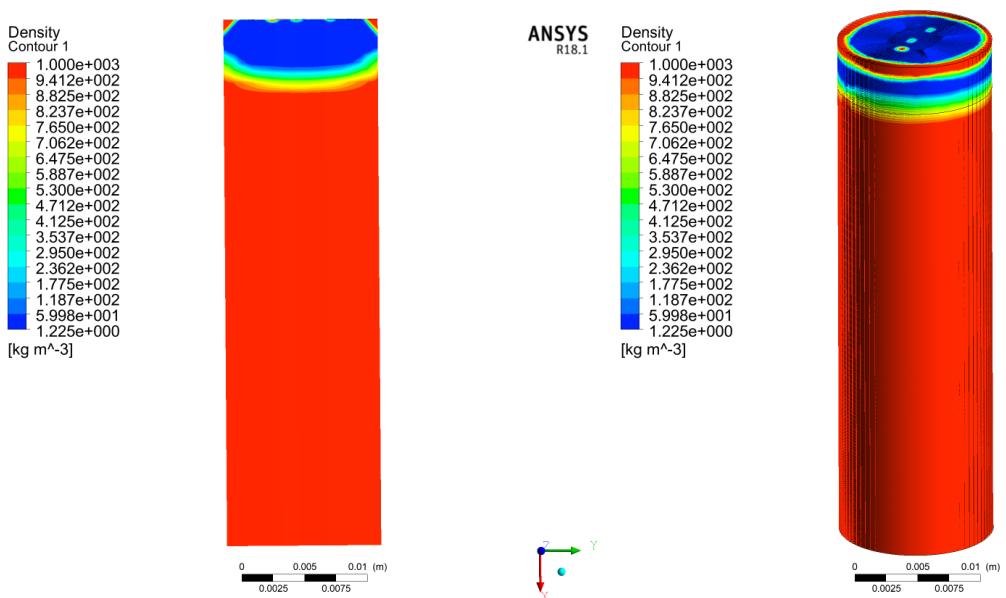

ANSYS

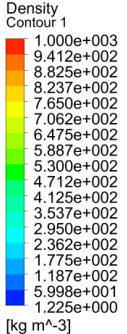

(f) Final State

(e) $t=3.0$ seconds

Figure 4.14 - A sectional density contour plot for a $5 \mathrm{~mL}$ cartridge spinning down to rest from 1.5 to 3 seconds. 

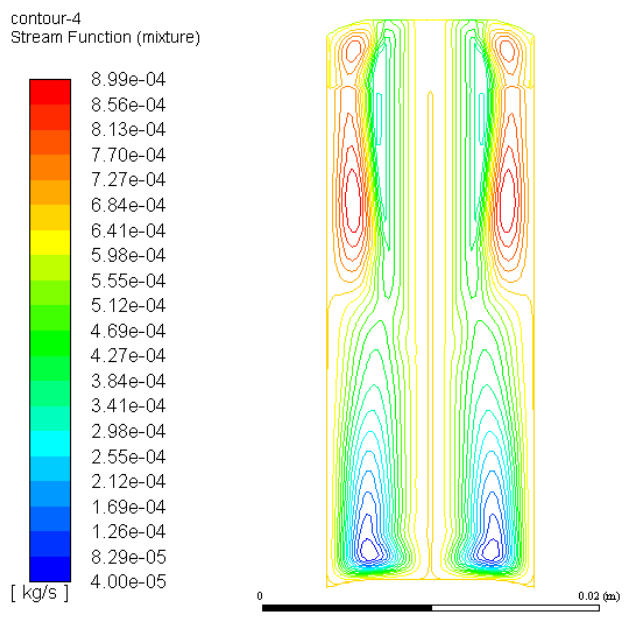

(a) 2-D Fluent
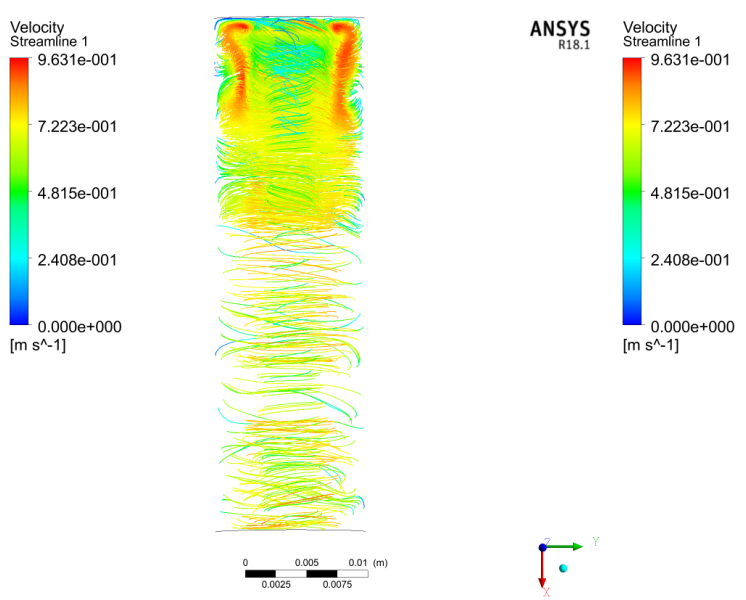

(b) 3-D Fluent Section View

(c) 3-D Fluent Full View

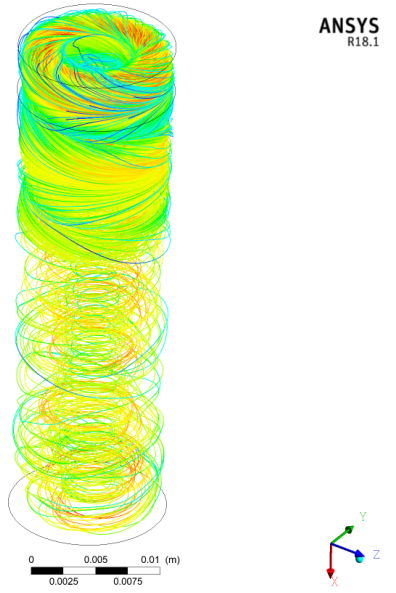

Figure 4.15 - Streamline comparison between the 2-D and 3-D Fluent model.

Streamlines were compared for a $5 \mathrm{~mL}$ cylinder at $3000 \mathrm{rpm}$ between a 2-D and 3-D Fluent model in Figure 4.15. In 2-D axisymmetric model, the streamlines are symmetric since they are mirrored as shown in Figure 4.15a. For a 3-D Fluent model, the streamlines do not appear to be symmetric as shown in Figure 4.15b. A perspective view of the $3-\mathrm{D}$ streamlines are shown in Figure $4.15 \mathrm{c}$. All the streamlines show a faster flow near the top of the container as shown in red. 


\subsubsection{Discussion}

The $5 \mathrm{~mL}$ cartridge Fluent models were compared with an experiment that shows favorable agreement as shown in Table 4.2. The 2-D and 3-D Fluent models had a percent error of 1.97 and $5.98 \%$, respectively. The mesh, iteration, and round-off error can be attributed to the difference between the experimental meniscus height. Also, the geometry of the models created in Fluent were simplified with the rounded corners, and can also be attributed as another source of error. In particular, the 3-D model assumed a perfectly cylinder geometry in comparison with the physical container geometry conducted during the experiments.

Upon taking a closer look in Figure 4.15 at the $5 \mathrm{~mL}$ cartridge at $20 \mathrm{cP}$, the streamlines show the TG vortices as mentioned by Neitzel [21] along the top containers. These TG vortices can cause the instabilities during spin-down as well as the bubble formation at the top of the container. The vortex breakdown is apparent as mentioned by Bhattacharyya [28] and Watson [23] where the bubble moves upward toward the top of the container as shown in Figure 4.12 4.14. It is apparent at high angular velocity and dynamic viscosity values cause the vortex breakdown to occur.

\section{$4.3 \quad 2.25 \mathrm{~mL}$ Syringe}

\subsubsection{Results}

The $2.25 \mathrm{~mL}$ container was used in studies in Fluent to find varying surface tension and angular velocity values. The surface tension was changed to three different values at $0.048,0.072$, and $0.096 \mathrm{~N} / \mathrm{m}$ for a $2.25 \mathrm{~mL}$ syringe spinning down to rest at 4000 rpm at 2.5 seconds.

Figure 4.16 shows there are subtle differences when changing the surface tension value. The yellow dashed boxes highlight the these differences. At $0.048 \mathrm{~N} / \mathrm{m}$, a 
yellow circle or presumed bubble is present in the liquid solution while a cyan colored semi-circle is present at the top of the container.

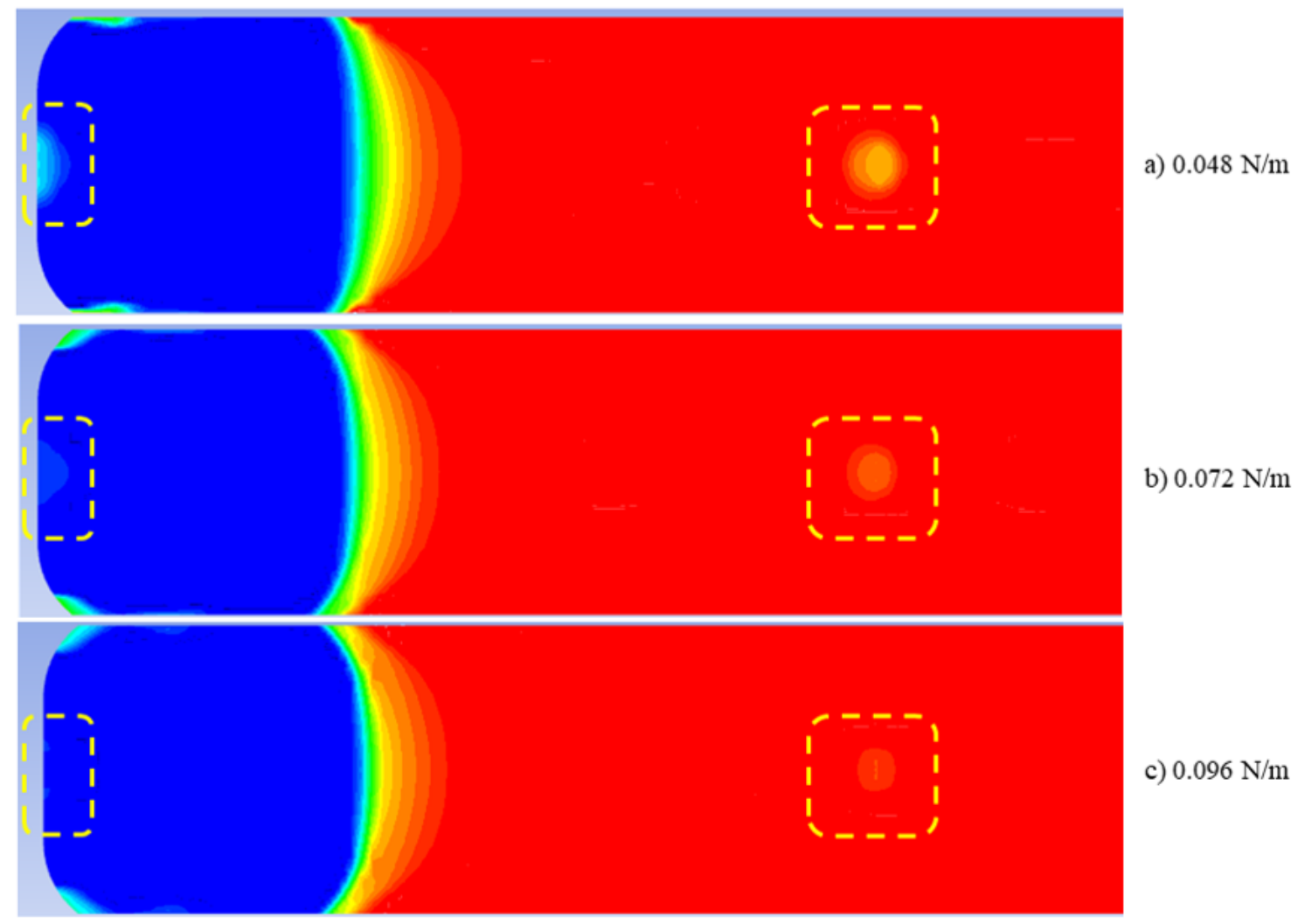

Figure 4.16 - Surface tension comparison of a $2.25 \mathrm{~mL}$ syringe at a) $0.048 \mathrm{~N} / \mathrm{m}, \mathrm{b}$ ) $0.072 \mathrm{~N} / \mathrm{m}$, and $0.096 \mathrm{~N} / \mathrm{m}$.

Angular velocity comparisons were done for a $2.25 \mathrm{~mL}$ syringe containing a dynamic viscosity of 39 centipoise. Since the surface tension for this particular solution is not known, the surface tension is assumed to be $0.072 \mathrm{~N} / \mathrm{m}$, which is the ideal surface tension for water.

\subsubsection{Discussion}

As shown in Figure 4.16, there are few differences when surface tension changes. When the surface tension values goes up, the bubbles at the top fade away. Since 
the surface tension value is increasing, there is an increase in resistance between the water molecules, thus preventing any liquid/air residue at the top. For the most part, surface tension does not have a profound effect on the instability during spin-down. Although Amgen syringe products do not container air gap, this study is to show the varying surface tension values having an effect on the Fluent models. 
Chapter 5

Conclusion

\subsection{Summary}

The goal of this project was to study the fluid movements and any bubble formation during Amgen's spin-stop method based on varying parameters of fill volume/air gap, dynamic viscosity, surface tension, and angular velocity. To accomplish this goal, a 2D and 3-D CFD model were simulated in Fluent using the VOF model. Experimental data and observations were also conducted at Amgen's lab to validate the results.

The 2-D model reduced the computational time to an hour to conduct many process scenarios and provided insight into the instabilities during spin-down to rest. The 3-D model showed subtle differences in streamline and density patterns in comparison to a 2-D axisymmetric model. However, there were no notable differences in the 3-D model from the 2-D model. The experiments further showed a favorable relationship with the meniscus height when comparing the 2-D and 3-D models with a percent difference of 1.97 and $5.98 \%$. Observations from the experiment showed comparable results in the instabilities during spin down, in which a bubble may separate from the meniscus to splash at the top of the container causing potential issues for quality check. Surface Tension did not have a profound effect on the outcomes of the simulation. Angular Velocity and dynamic viscosity provided the biggest changes in the results with the spin-down instabilities and vortex breakdown. 
In summary, the CFD models provided insight into the following:

1. The meniscus height dewetts at the bottom angular velocities of $5000 \mathrm{rpm}$ or higher for the $1 \mathrm{~mL}$ syringe and $5 \mathrm{~mL}$ cartridge.

2. Spin-down to rest shows instabilities for high dynamic viscosity and angular velocity values and led to a vortex breakdown, creating bubbles at the top of the containers.

3. 2-D models in Fluent provided accurate results with less computational time than 3-D models.

\subsection{Future Work}

This is only the start for Amgen's use of CFD models to investigate the various drug product simulations for optimal quality inspections. By doing initial study models for the $1 \mathrm{~mL}$ syringe, $5 \mathrm{~mL}$ cartridge, and $2.25 \mathrm{~mL}$ syringe, a pathway for work can be continued to further understand the proper use of the spin-stop method. Further research and development can be done by refining the Fluent models to replicate physical description of the containers such as the top portion of the containers for cap and needle for a cartridge and syringe, respectively.

A focus on the spin-down studies can provide more insight into the vortex breakdown for bubbles at the top of the containers. Since varying start-up times were only done for spin-up, a study for varying ramp down times should be conducted for spin down to reduce the instabilities and vortex breakdown. Also, contact angle did play a role in the sensitivity of the meniscus height during the validation model and should be further explored. 


\section{BIBLIOGRAPHY}

[1] T. W. Randolph, E. Schiltz, D. Sederstrom, D. Steinmann, O. Mozziconacci, C. Schöneich, E. Freund, M. S. Ricci, J. F. Carpenter, and C. S. Lengsfeld, "Do not drop: mechanical shock in vials causes cavitation, protein aggregation, and particle formation.," Journal of pharmaceutical sciences, vol. 104, pp. 602-11, Feb. 2015.

[2] R. A. Ibrahim, Liquid Sloshing Dynamics: Theory and Applications. Cambridge University Press, 2005.

[3] H. P. Greenspan and L. N. Howard, "On a time-dependent motion of a rotating fluid," Journal of Fluid Mechanics, vol. 17, p. 385, Nov. 1963.

[4] H. Greenspan, The Theory of Rotating Fluids. Cambridge University Press, 1968.

[5] F. Yan and B. Farouk, "Numerical Simulations of Flows Inside a Partially Filled Centrifuge," Journal of Fluids Engineering, vol. 125, p. 1033, Nov. 2003.

[6] V. A. Lubarda, "The shape of a liquid surface in a uniformly rotating cylinder in the presence of surface tension," Acta Mechanica, vol. 224, pp. 1365-1382, July 2013.

[7] E. H. Wedemeyer, "The unsteady flow within a spinning cylinder," Journal of Fluid Mechanics, vol. 20, p. 383, Nov. 1964.

[8] K. Stewartson, "On almost rigid rotations," Journal of Fluid Mechanics, vol. 3, p. 17, Oct. 1957. 
[9] J. M. Hyun, F. Leslie, W. W. Fowlis, and A. Warn-Varnas, "Numerical solutions for spin-up from rest in a cylinder," Journal of Fluid Mechanics, vol. 127, pp. 263-281, 1983.

[10] A. Warn-Varnas, W. W. Fowlis, S. Piacsek, and S. M. Lee, "Numerical solutions and laser-Doppler measurements of spin-up," Journal of Fluid Mechanics, vol. 85, p. 609, April 1978.

[11] E. R. Benton, "Vorticity dynamics in spinup from rest," The Physics of Fluids, vol. 22 , no. 7 , pp. 1250-1251, 1979.

[12] H. Schlichting, Boundary Layer Theory. New York: McGraw-Hill, 7th ed., 1979.

[13] S. Choi, J. W. Kim, and J. M. Hyun, "Transient Free Surface Shape in an Abruptly Rotating, Partially Filled Cylinder," Journal of Fluids Engineering, vol. 111, p. 439, Dec. 1989.

[14] M. A. Shadday, R. J. Ribando, and J. J. Kauzlarich, "Flow of an incompressible fluid in a partially filled, rapidly rotating cylinder with a differentially rotating endcap," Journal of Fluid Mechanics, vol. 130, p. 203, May 1983.

[15] W. B. Watkins and R. G. Hussey, "Spin-up from rest in a cylinder," Physics of Fluids, vol. 20, no. 10, p. 1596, 1977.

[16] J. W. Miles and F. K. Ball, "On free-surface oscillations in a rotating paraboloid," Journal of Fluid Mechanics, vol. 17, no. 2, pp. 257-266, 1963.

[17] J. W. Miles, "Free-surface oscillations in a slowly rotating liquid," Journal of Fluid Mechanics, vol. 18, p. 187, feb 1964.

[18] J. W. Miles and B. A. Troesch, "Surface Oscillations of a Rotating Liquid," Journal of Applied Mechanics, vol. 28, p. 491, Dec. 1961. 
[19] N. Gerber, "Properties of Rigidly Rotating Liquids in Closed Partially Filled Cylinders," Journal of Applied Mechanics, vol. 42, p. 734, Sep. 1975.

[20] G. F. Homicz and N. Gerber, "Numerical Model for Fluid Spin-Up From Rest in a Partially Filled Cylinder," Journal of Fluids Engineering, vol. 109, p. 194, June 1987.

[21] G. P. Neitzel and S. H. Davis, "Centrifugal instabilities during spin-down to rest in finite cylinders. Numerical experiments," Journal of Fluid Mechanics, vol. 102, p. 329, Jan. 1981.

[22] G. P. Neitzel and S. H. Davis, "Energy stability theory of decelerating swirl flows," Citation: The Physics of Fluids, vol. 23, p. 432, 1980.

[23] J. P. Watson and G. P. Neitzel, "Numerical evaluation of a vortex-breakdown criterion," Physics of Fluids, vol. 8, p. 3063, June 1998.

[24] G. A. Euteneuer, "Die entwicklung von längswirbeln in zeitlich anwachsenden grenzschichten an konkaven wänden," Acta Mechanica, vol. 13, pp. 215-223, Sep 1972.

[25] X. Cui, "Numerical simulations of the generation of Taylor-Görtler vortices during spin-down to rest in finite-length cylinders," Computers and Fluids, vol. 33, no. 4, pp. 603-621, 2004.

[26] M. C. Kim and C. Kyun Choi, "The onset of Taylor-Görtler vortices during impulsive spin-down to rest," Chemical Engineering Science, vol. 61, no. 19, pp. 6478-6485, 2006.

[27] D. M. Mathis and G. P. Neitzel, "Experiments on impulsive spin-down to rest," Citation: The Physics of Fluids, vol. 28, p. 1535, 1985. 
[28] S. Bhattacharyya and A. Pal, "Generation (or Degeneration) of a Separation Bubble in a Liquid-Filled Cylinder Through Spin-Up (or Spin-Down) Process," Journal of Applied Mechanics, vol. 66, p. 1023, Dec. 1999.

[29] ANSYS Theory Guide 19.1. Pennsylvania: ANSYS Inc., 2018. 
Appendix A

MATLAB Code

\section{Surface Tension Meniscus Height}

$\%$ Calculate height of meniscus for rigid body rotation with and without \%Created by Professor Kim Shollenberger, 2018

$\%$ Edited by Matthew Ichinose, 2018

$\%$ Surface tension using V. Lubarda model

clear, clc

$\%$ Define parameters:

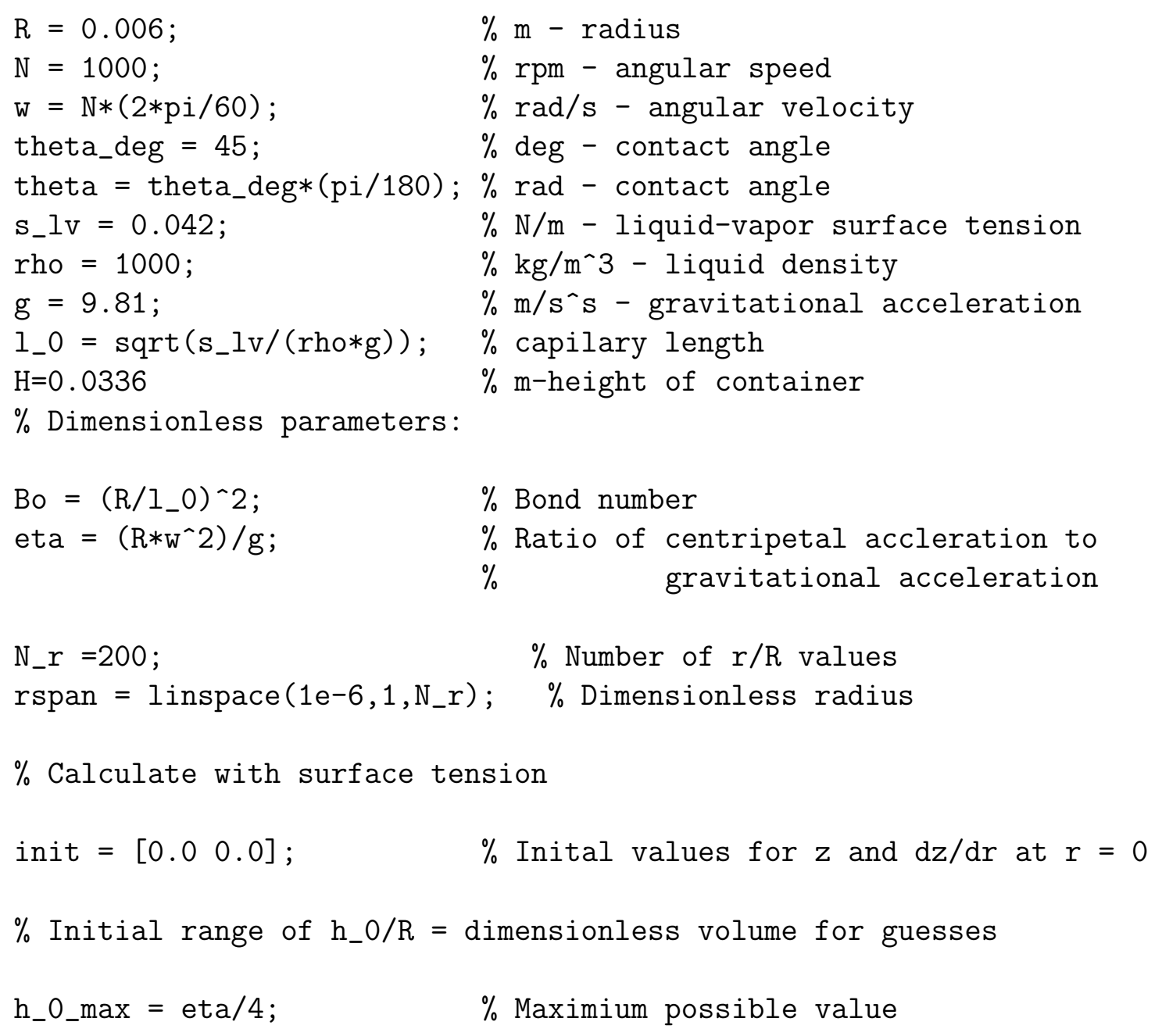




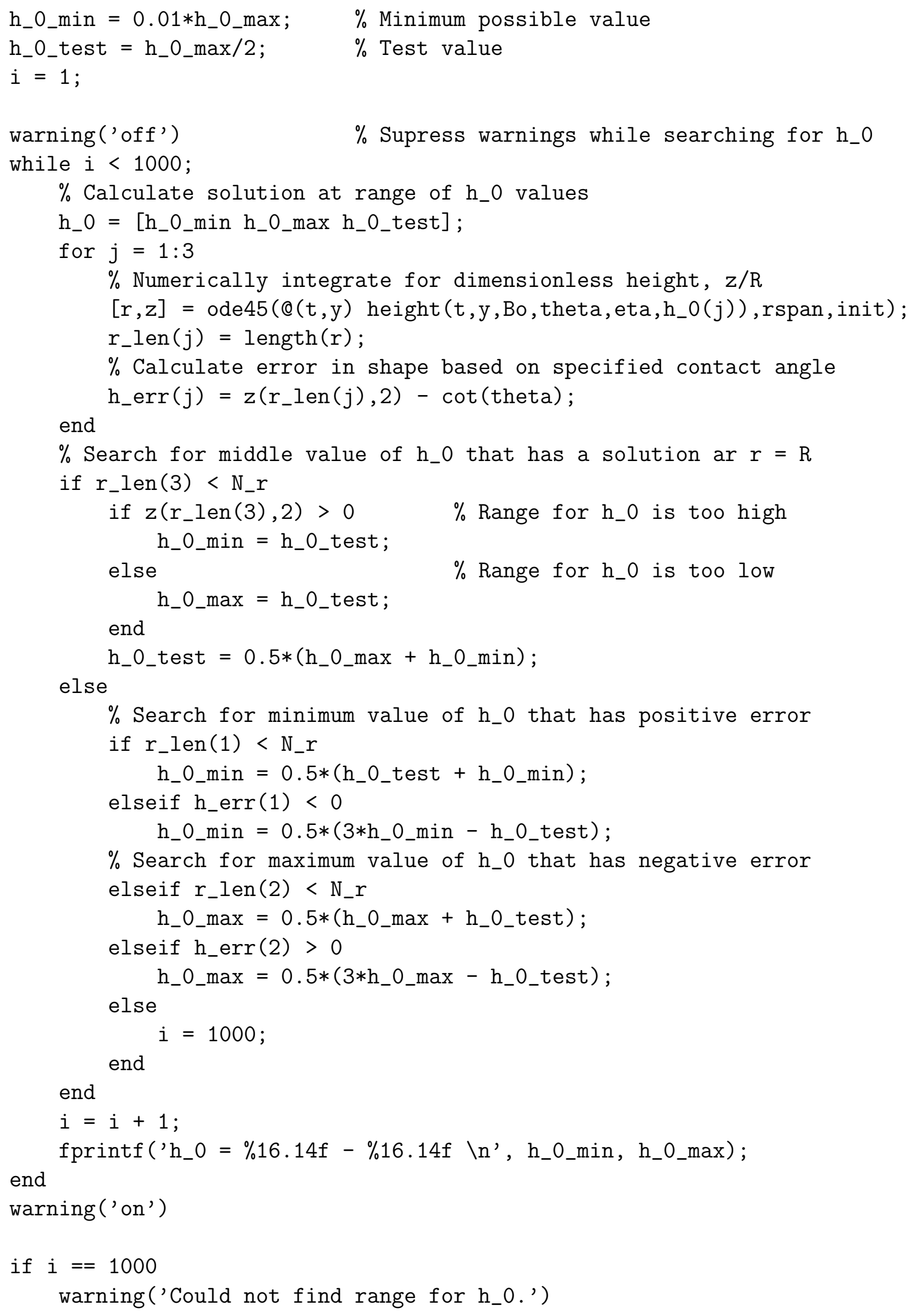


else

$\%$ Refine calculatioin of $\mathrm{h}_{-} 0$ by minimizing error in contact angle

N_max $=5 ; \quad \%$ Number of profiles to use for each error test

h_0 = linspace (h_0_min, h_0_max, N_max);

markers $=\left[{ }^{\prime}-k^{\prime},,^{\prime}--k^{\prime},{ }^{\prime}-. k^{\prime},,^{\prime}: k^{\prime}, \ldots\right.$

'-b', ,--b', '-. b', , : b', ..

'-r', '--r', '-.r', ': $\left.r^{\prime}\right]$;

figure (1);

for $j=1: 5 \quad \%$ Number of error refinements

subplot $(2,1,1$, 'replace');

hold on;

for $i=1:$ N_max

$\%$ Numerically integrate for dimensionless height, $z / R$

$[r, z]=\operatorname{ode} 45(@(t, y)$ height $(t, y$, Bo, theta, eta, h_0(i)), rspan, init);

$\%$ Calculate error in shape based on specified contact angle $\operatorname{error}(i, j)=z\left(N \_r, 2\right)-\cot ($ theta $)$;

plot $(r, z(:, 1), \operatorname{char}(\operatorname{markers}(i)), \ldots$

'LineWidth', 2, 'DisplayName', $\operatorname{string}(\operatorname{error}(i, j)))$;

end

title (['Bo $=$ ', $\operatorname{char}(\operatorname{string}(\mathrm{Bo})), \ldots$

', $\backslash$ eta $=$ ', $\operatorname{char}(\operatorname{string}($ eta $)), \ldots$.

', $\backslash$ theta $=$ ', $\operatorname{char}(\operatorname{string}($ theta_deg $))$, ' deg'] $)$;

xlabel ('r/R');

ylabel ('z/R');

$\mathrm{x} \lim \left(\left[\begin{array}{ll}0 & 1.0\end{array}\right]\right)$;

$\% \operatorname{ylim}\left(\left[\begin{array}{ll}0 & 8.0\end{array}\right]\right)$;

lgd = legend ('show', 'Location', 'northwest') ;

title(lgd, 'Error')

hold off

subplot $(2,1,2$, 'replace')

$\operatorname{plot}\left(h_{-} 0, \operatorname{error}(:, j),{ }^{\prime}--k *^{\prime}\right)$;

xlabel ('h_0');

ylabel ('error');

$\%$ Refine range for $h_{-} 0 / R$ guess using error verus $h_{-} 0$ curve h_0_min_new $=\max \left(\right.$ interp1 $\left(\operatorname{error}(:, j), h_{-} 0,1 / j,{ }^{\prime}\right.$ spline' $), h_{-}$__min $)$;

h_0_max_new $=\min \left(\right.$ interp1 $\left(\operatorname{error}(:, j), h_{-} 0,-1 / j,{ }^{\prime}\right.$ spline'),$h_{-}$0_max $)$; 


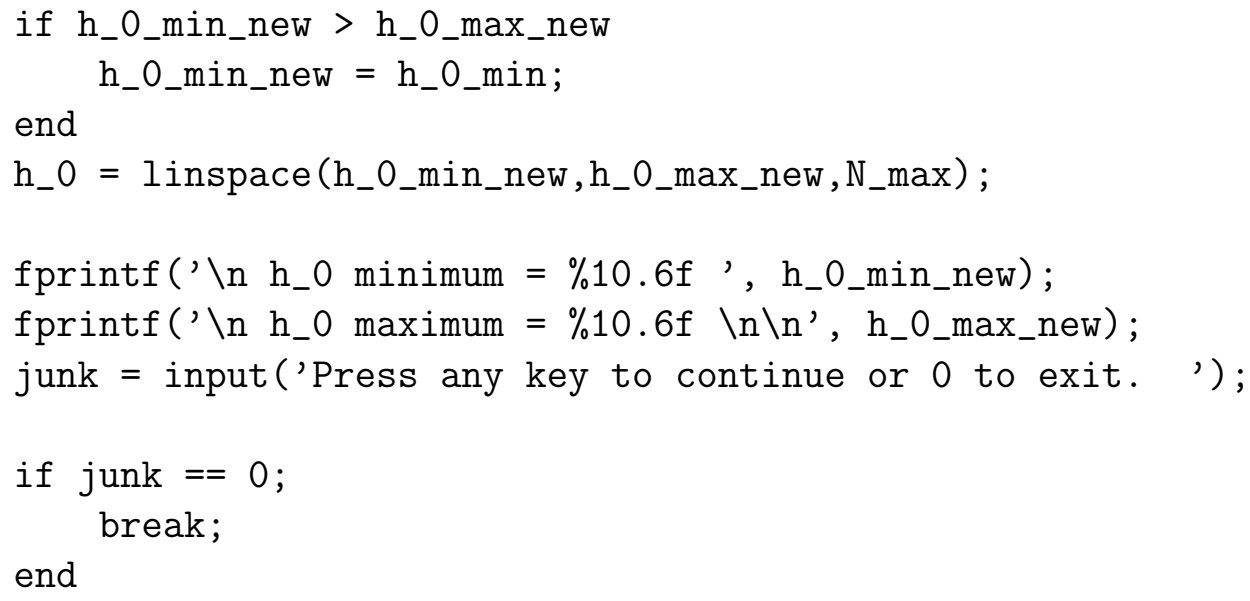

end

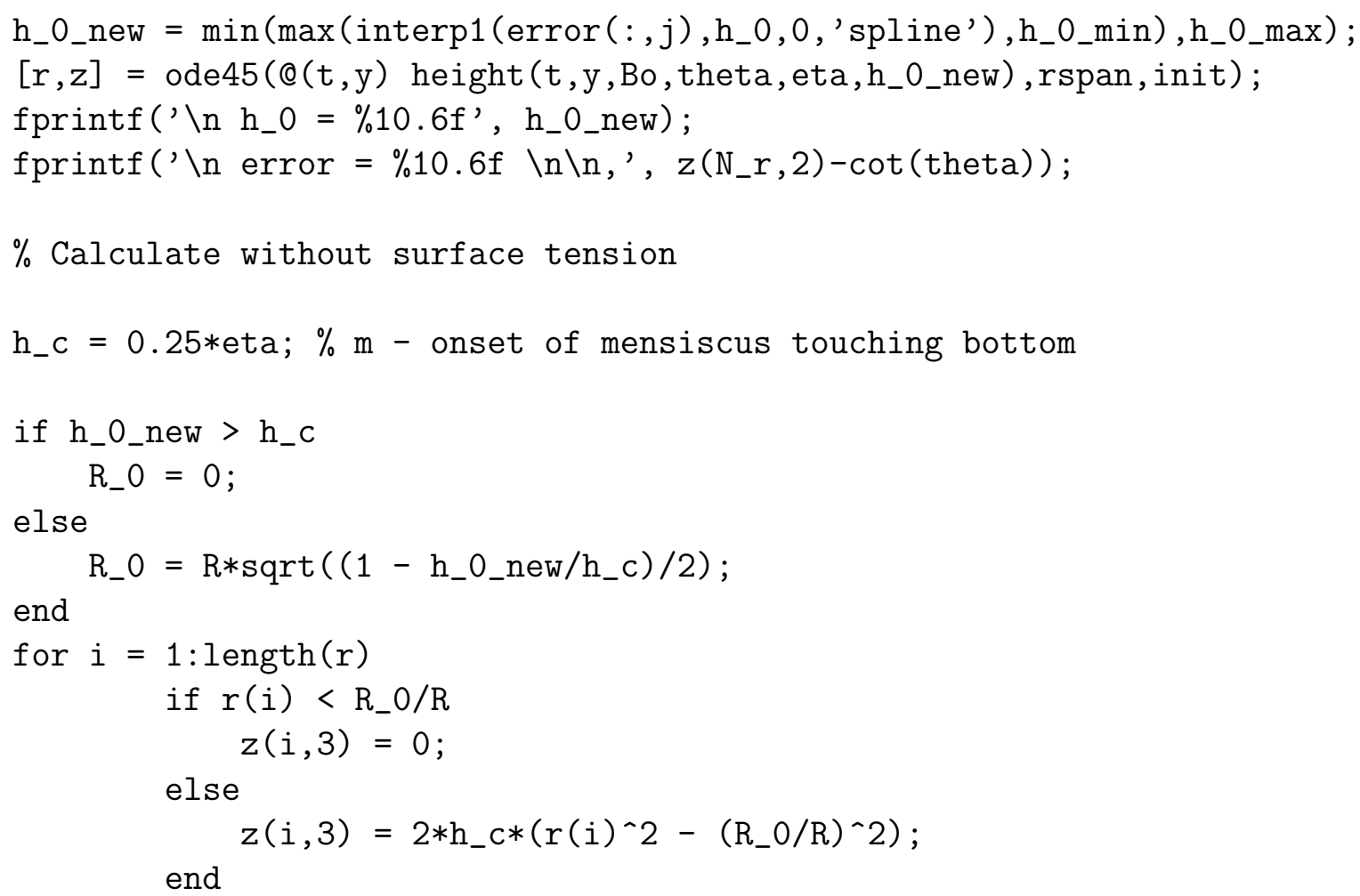

end

figure (2);

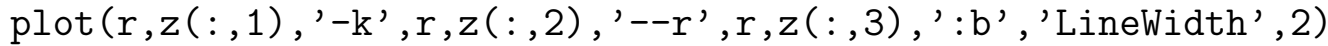

title $([$ 'Bo $=$ ', $\operatorname{char}(\operatorname{string}(\mathrm{Bo})), \ldots$

,, eta $=$ ', $\operatorname{char}(\operatorname{string}($ eta $)), \ldots$

', $\backslash$ theta $=$ ', $\operatorname{char}($ string $($ theta_deg $))$, 'deg'] $)$;

xlabel ('r/R');

ylabel ('z/R');

legend('z/R with \sigma','dz/dr','z/R without \sigma',... 
'Location', 'northwest')

figure (3);

plot $\left(r, z(:, 1),{ }^{\prime}-k \text { ', } r, z(:, 3),\right)^{\prime}: b^{\prime}$, 'LineWidth', 2)

title $([$ 'Bo $=$ ', $\operatorname{char}(\operatorname{string}(\mathrm{Bo})), \ldots$

', $\backslash$ eta $=$ ', $\operatorname{char}(\operatorname{string}($ eta $)), \ldots$

', $\backslash$ theta $=$ ', $\operatorname{char}($ string $($ theta_deg $))$, ' deg'] $)$;

xlabel ('r/R');

ylabel ('z/R');

legend('z/R with \sigma', 'z/R without \sigma',...

'Location', 'northwest')

end

$\mathrm{H}=$

0.0336

$\mathrm{h}_{-} 0=0.83839656822030-1.67679313644060$

$\mathrm{h}_{-} 0=0.83839656822030-1.25759485233045$

$\mathrm{h}_{-} 0=1.04799571027537-1.25759485233045$

$\mathrm{h}_{-} 0=1.15279528130291-1.25759485233045$

$\mathrm{h}_{-} 0=1.15279528130291-1.20519506681668$

$\mathrm{h}_{-} 0=1.17899517405980-1.20519506681668$

$\mathrm{h}_{-} 0=1.19209512043824-1.20519506681668$

$\mathrm{h}_{-} 0=1.19209512043824-1.19864509362746$

$\mathrm{h}_{-} 0=1.19373261373554-1.19864509362746$

$\mathrm{h}_{-} 0=1.19455136038420-1.19864509362746$

$\mathrm{h}_{-} 0=1.19496073370852-1.19864509362746$

$\mathrm{h}_{-} 0=1.19516542037068-1.19864509362746$

$\mathrm{h}_{-} 0=1.19506307703960-1.19864509362746$

$\mathrm{h}_{-} 0=1.19521659203623-1.19864509362746$

$\mathrm{h}_{-} 0=1.19513983453791-1.19864509362746$

$\mathrm{h}_{-} 0=1.19502469829045-1.19864509362746$

$\mathrm{h}_{-} 0=1.19519740266165-1.19864509362746$

$\mathrm{h}_{-} 0=1.19511105047605-1.19864509362746$

$\mathrm{h}_{-} 0=1.19498152219765-1.19864509362746$

$\mathrm{h}_{-} 0=1.19517581461525-1.19864509362746$

$\mathrm{h}_{-} 0=1.19507866840645-1.19864509362746$

$\mathrm{h}_{-} 0=1.19507866840645-1.19700760033015$

$\mathrm{h}_{-} 0=1.19507866840645-1.19618885368150$

$\mathrm{h}_{-} 0=1.19507866840645-1.19618885368150$

h_0 minimum $=1.195079$

h_0 maximum $=1.194964$ 
Error using input

Cannot call INPUT from EVALC.

Error in Meniscus_height (line 134)

junk $=$ input ('Press any key to continue or 0 to exit. ') ;

\section{Height Function File}

function dydt $=$ height $(t, y$, Bo, theta, eta,h_0 $)$

$\%$ Evaluate the derivatives for $\mathrm{y} 1=\mathrm{z}$ and $\mathrm{y} 2=\mathrm{dz} / \mathrm{dr}$

$\operatorname{dydt}=\operatorname{zeros}(2,1)$

$\operatorname{dydt}(1)=\mathrm{y}(2)$

$\operatorname{dydt}(2)=-(1+y(2) \wedge 2) * y(2) / t+\left(1+y(2)^{\wedge} 2\right)^{\wedge}(3 / 2) * \ldots$

$\left(2 * \cos (\right.$ theta $\left.)+\operatorname{Bo} *\left(y(1)-\mathrm{h}_{-} 0+0.25 * \operatorname{eta} *\left(1-2 * t .{ }^{\wedge} 2\right)\right)\right)$;

Not enough input arguments.

Error in height (line 6)

$\operatorname{dydt}(1)=y(2)$;

\section{Acceleration and Wall Shear Stress}

$\%$ Calculate the velocity profiles and shear stresses versus time

$\%$ for the fluid in an infinite cylinder for:

$\%$

$\% \quad$ (1) step change in velocity at $t=0$

$\%$ (2) linear increase in velocity for $t=0$ to $t_{-} 0$

$\%$ Created by Dr. Kim Shollenberger in 2018.

$\%$ Edited by Matthew Ichinose.

clear, clc

$$
\begin{aligned}
& \mathrm{a}=0.0032 ; \quad \% \mathrm{~m} \text {, radius } \\
& \text { t_0 }=0.0025 ; \quad \% \mathrm{~s} \text {, time for velocity change } \\
& \text { omega }=314 ; \% \mathrm{rad} / \mathrm{s} \text {, angular velocity } \\
& \mathrm{nu}=1.0 \mathrm{e}-6 ; \quad \% \mathrm{~m}^{\wedge} 2 / \mathrm{s}, \text { kinematic viscosity of water } \\
& \mathrm{g}=9.81 ; \quad \% \mathrm{~m} / \mathrm{s}^{\wedge} 2 \text {, gravitational accleration } \\
& \text { Re }=\text { omega } * a^{\wedge} 2 / \mathrm{nu} ; \quad \% \text { Reynolds number } \\
& \text { Fr }=\text { omega } * \text { sqrt }(\mathrm{a} / \mathrm{g}) ; \% \text { Froude number }
\end{aligned}
$$




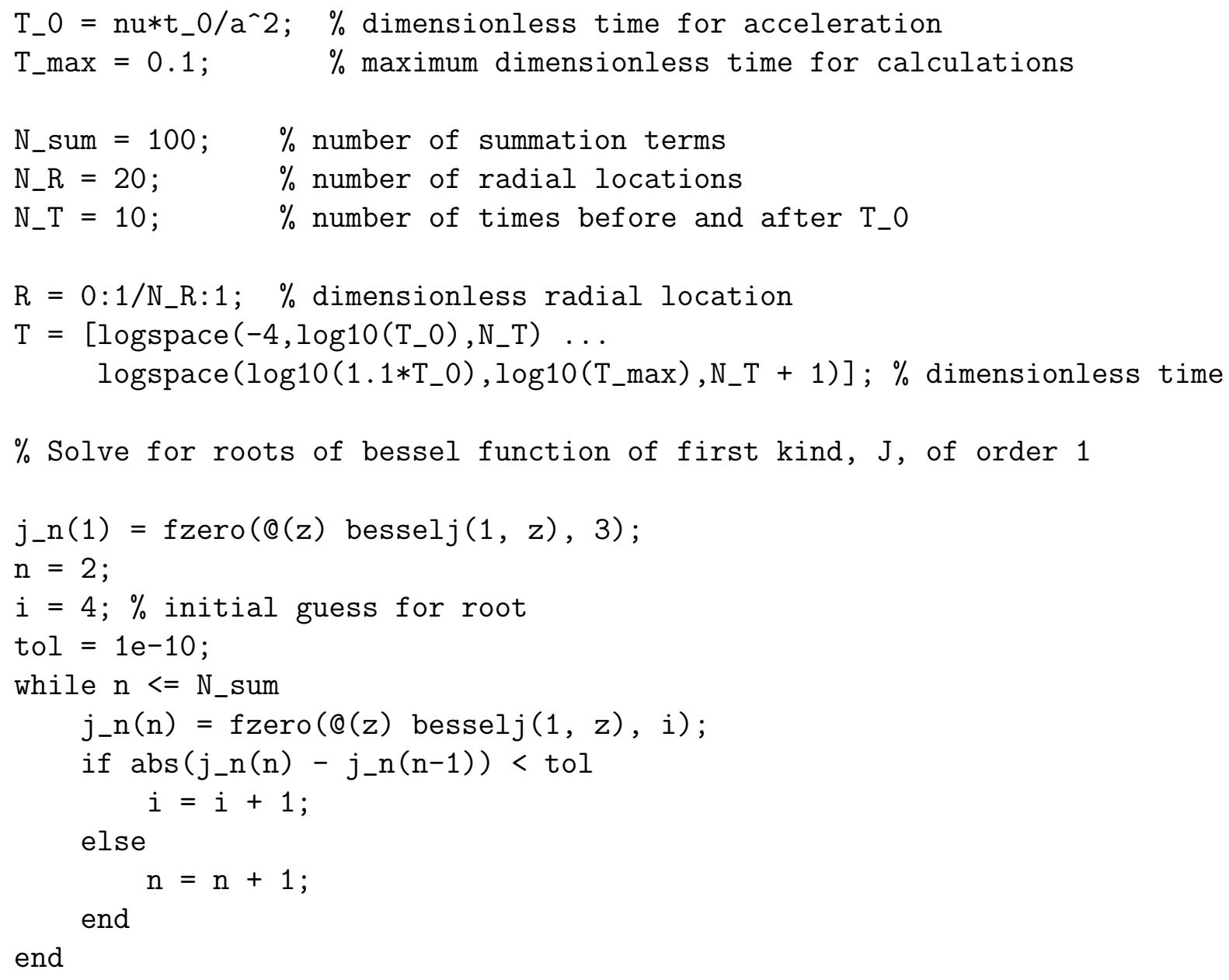


end

end

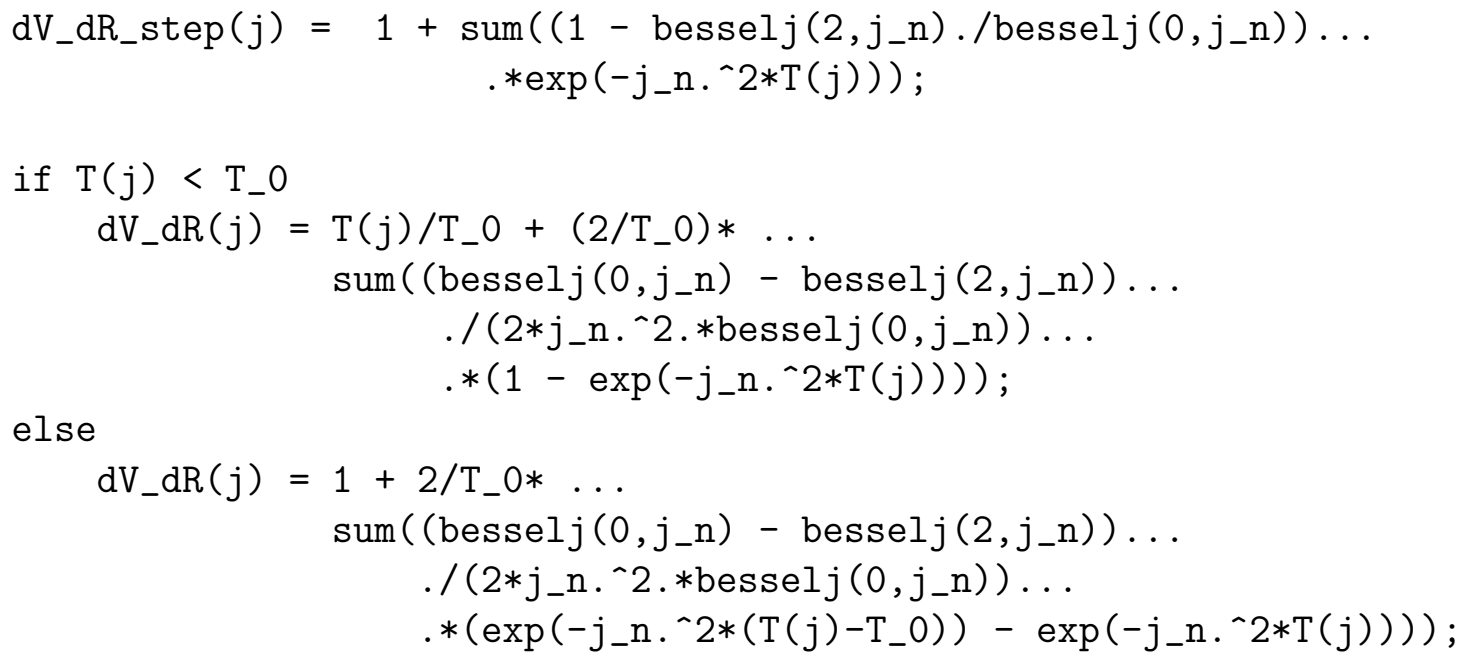

else

end

end

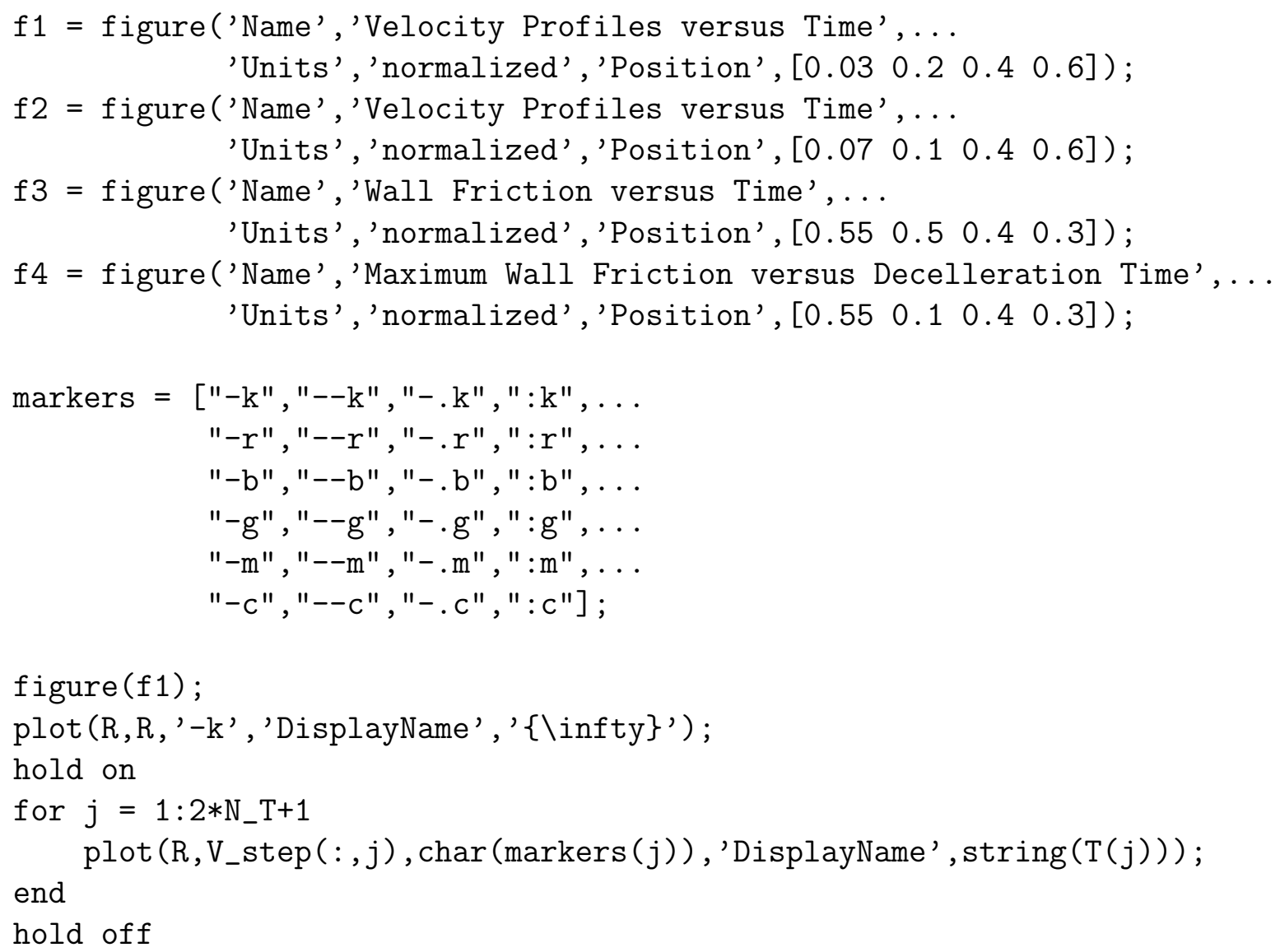




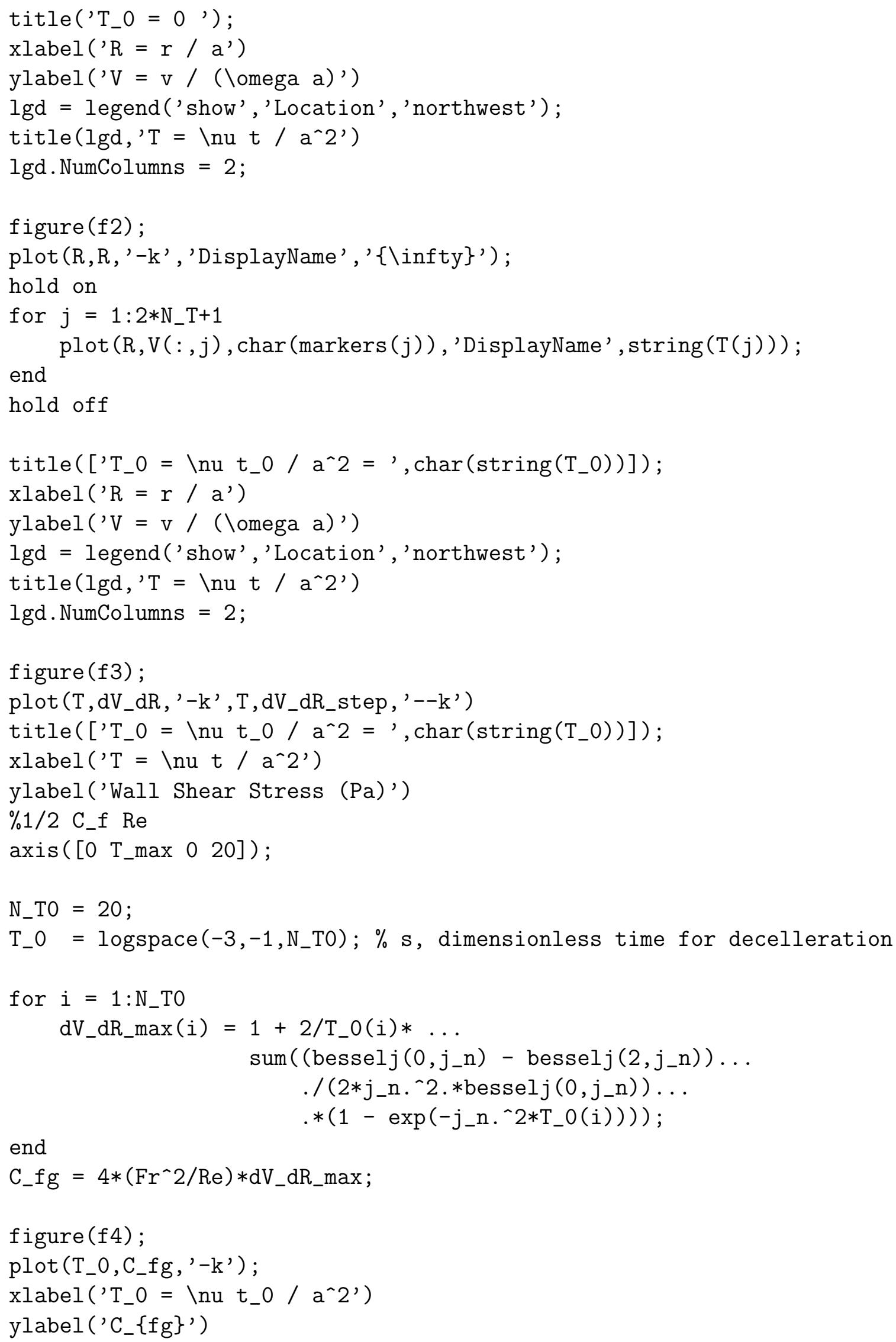

end

C_fg $=4 *\left(\mathrm{Fr}^{\wedge} 2 / \mathrm{Re}\right) * d V_{-} d R_{-} \max$;

figure (f4);

plot (T_0,C_fg, '-k');

xlabel ('T_0 = \nu t_0 / $\mathrm{a}^{\wedge} \mathrm{2}^{\prime}$ ')

ylabel ('C_\{fg\}') 


\section{Appendix B}

\section{Sample List for Experiment}

This section details the created samples for the experiments. A list of the samples is shown in Table B.1. C-1 through C-8 were different samples for a $5 \mathrm{~mL}$ cartrdige. All samples were created in a non-clean environment, therefore there were fibers and micro bubble existing in the liquid domain. S-1 through S-7 were samples for a 2.25 mL syringe. S-8 was a sample for a $1 \mathrm{~mL}$ syringe. $\mathrm{S}-8$ was used to compare values with the analytical validation models at $1800 \mathrm{rpm}$ at $1 \mathrm{cP}$ with an air gap of 4.14 $\mathrm{mm}$.

When a sample was tested inside the SpinCAM, observations were noted for initial conditions existing in the container such as micro bubbles or fibers. C-1 and C3 through C-6 had micrometer pieces of fibers in the liquid due to contamination. C-7 and C-8 had micro bubbles and a piece of fiber on the vertical walls of the container. S-1, S-2, and S-4 through S-8 had micro bubbles and fibers present in the container. C-2 and S-3 were created from a clean room environment; therefore, there were no initial conditions present. 
Table B.1 - Sample list for experimental setup with air gap and viscosity values.

\begin{tabular}{|c|c|c|}
\hline Sample No. & Air Gap (mm) & Viscosity (cP) \\
\hline C-1 & 3.72 & 5 \\
\hline $\mathrm{C}-2$ & 2.50 & 5 \\
\hline $\mathrm{C}-3$ & 5.17 & 5 \\
\hline $\mathrm{C}-4$ & 7.54 & 5 \\
\hline $\mathrm{C}-5$ & 8.24 & 10 \\
\hline $\mathrm{C}-6$ & 3.08 & 10 \\
\hline $\mathrm{C}-7$ & 8.17 & 10 \\
\hline $\mathrm{C}-8$ & 5.25 & 1 \\
\hline S-1 & 3.40 & 10 \\
\hline S-2 & 2.95 & 37 \\
\hline S-3 & 0.00 & 37 \\
\hline S-4 & 4.48 & 10 \\
\hline S-5 & 0.00 & 10 \\
\hline S-6 & 1.40 & 37 \\
\hline S-7 & 0.00 & 10 \\
\hline S-8 & 4.14 & 1 \\
\hline
\end{tabular}

